\title{
Identification of differentially expressed genes and signaling pathways in gestational diabetes mellitus by integrated bioinformatics analysis
}

Basavaraj Vastrad ${ }^{1}$, Chanabasayya Vastrad ${ }^{* 2}$

1. Department of Biochemistry, Basaveshwar College of Pharmacy, Gadag, Karnataka 582103, India.

2. Biostatistics and Bioinformatics, Chanabasava Nilaya, Bharthinagar, Dharwad 580001, Karanataka, India.

* Chanabasayya Vastrad

channu.vastrad@gmail.com

Ph: +919480073398

Chanabasava Nilaya, Bharthinagar,

Dharwad 580001 , Karanataka, India 


\begin{abstract}
Gestational diabetes mellitus (GDM) is a metabolic disorder during pregnancy. Numerous biomarkers have been identified that are linked with the occurrence and development of GDM. The aim of this investigation was to identify differentially expressed genes (DEGs) in GDM using a bioinformatics approach to elucidate their molecular pathogenesis. GDM associated expression profiling by high throughput sequencing dataset (GSE154377) was obtained from Gene Expression Omnibus (GEO) database including 28 normal pregnancy samples and 33 GDM samples. DEGs were identified using DESeq2. The gene ontology (GO) and REACTOME pathway enrichments of DEGs were performed by $\mathrm{g}$ :Profiler. Protein-protein interaction (PPI) networks were assembled with Cytoscape software and separated into modules using the PEWCC1 algorithm. MiRNA-hub gene regulatory network and TF-hub gene regulatory network were performed with the miRNet database and NetworkAnalyst database. Receiver Operating Characteristic (ROC) analyses was conducted to validate the hub genes. A total of 953 DEGs were identified, of which 478 DEGs were up regulated and 475 DEGs were down regulated. Furthermore, GO and REACTOME pathway enrichment analysis demonstrated that these DEGs were mainly enriched in multicellular organismal process, cell activation, formation of the cornified envelope and hemostasis. TRIM54, ELAVL2, PTN, KIT, BMPR1B, APP, SRC, ITGA4, RPA1 and ACTB were identified as key genes in the PPI network, miRNA-hub gene regulatory network and TF-hub gene regulatory network. TRIM54, ELAVL2, PTN, KIT, BMPR1B, APP, SRC, ITGA4, RPA1 and ACTB in GDM were validated using ROC analysis. This investigation provides further insights into the molecular pathogenesis of GDM, which might facilitate the diagnosis and treatment of GDM.
\end{abstract}

Keywords: gestational diabetes mellitus; hub gene; gene expression omnibus; bioinformatics; protein-protein interaction network 


\section{Introduction}

Gestational diabetes mellitus (GDM) has emerged as most common medical complications of pregnancy, but current treatments are still suboptimal [1]. The number of cases GDM is rising worldwide and it has become a key health concern [2]. There are several important risk factors for GDM, such as advanced maternal age [3], family history of diabetes [4], obesity [5], cigarette smoking [6], genetics factors [7], hypertension and type 2 diabetes [8], and cardiovascular diseases [9]. However, GDM is a complex disorder and its biology remains poorly understood. Therefore, mining key biomarkers linked with GDM occurrence and advancement is essential for GDM early diagnosis, prognosis and treatment.

The GDM is an extremely complicated pathophysiological process and controlled by various genes and signaling pathways. Genes such as PVT1 [10], IL6, IL-10, and TNF- $\alpha$ [11], CAPN10 [12], ADCYAP1 [13] and ABHD5 [14] are associated with GDM. Signaling pathways such as PI3K/AKT signaling pathway [15], AMPK signaling pathway [16], vitamin D signaling pathways [17], TLR4/NF-kappaB Signaling Pathway [18] and AGEs-RAGE signaling pathway [19] are linked with GDM. Recently, next-generation sequencing (NGS) technology is extensively applied in clinical investigation, with the marked gain relying on its capacity to together determine the expression data of massive genes at a time [20]. The public database Gene Expression Omnibus (GEO) (http://www.ncbi.nlm.nih.gov/geo/) [21] are powerful tools used to screen the differentially expressed genes (DEGs) generated from NGS data corresponding to the progression of GDM [22]. Therefore, there are still many related genes to be identified, which will help us better understand the molecular pathogenesis of GDM and facilitate the discovery of new diagnostic biomarkers or therapeutic target.

In the current investigation, we tested integrated bioinformatics analysis for the identification of DEGs as potential biomarkers of GDM based on the expression profiling by high throughput sequencing from the GEO database. These analysis might provide possible perspectives for the progression and development of GDM as well as identification potential candidate genes for GDM diagnose or treatment. 


\section{Materials and methods}

\section{Data resources}

The NGS data used in this investigation were acquired from GEO database, which is a public repository containing the expression profiling by high throughput sequencing data submitted by research institutions around the world. We chose the expression profiling by high throughput sequencing data GSE154377 [23] from the GEO database. The NGS data of GSE154377 was consisted with 28 normal pregnancy samples and 33 GDM samples based on the platform of GPL20301 Illumina HiSeq 4000 (Homo sapiens).

\section{Identification of DEGs}

DESeq2 [24] packages of $\mathrm{R}$ Bioconductor (http://www.bioconductor.org/) was applied to significance analysis of DEGs between normal pregnancy samples and GDM samples. The log-fold change (FC) in expression and adjusted P-values (adj. $\mathrm{P})$ were resolved. The criterion of statistical significance was adj. $\mathrm{P}$ value $<0.05$, $|\log 2(\mathrm{FC})|>1.73$ for up regulated genes and $|\log 2(\mathrm{FC})|>-0.69$ for down regulated genes. A heatmap of the DEGs was produced using gplots in R software. All significant DEGs were shown in a volcano plot generated using ggplot 2 in $\mathrm{R}$ software.

\section{Gene Ontology (GO) and REACTOME pathway enrichment analysis of DEGs}

Gene Ontology (GO) (http://www.geneontology.org) [25] is a suitable technique to classify gene expression and its properties, including biological process (BP), cellular component (CC) and molecular function (MF), which provide extensive functional annotation tools for researchers to integrate key genes with a specific function. REACTOME (https://reactome.org/) [26] is an online pathway database that collects information on genomic, biochemical, and enzymatic pathways. The DEGs were mapped to the REACTOME pathway database and the significantly related pathways were screened. We could use g:Profiler (http://biit.cs.ut.ee/gprofiler/) [27] is an online functional annotation tool to visualize the DEGs enrichment of $\mathrm{BP}, \mathrm{MF}, \mathrm{CC}$ and pathways ( $\mathrm{P} \square<\square 0.05$ ).

\section{Construction of the PPI network and module analysis}


IID interactome (Integrated Interactions Database) (http://iid.ophid.utoronto.ca/) [28] was used to construct PPI network. The identified DEGs were input into IID interactome to unravel a potential PPI network. Afterwards, a PPI network was constructed and visualized by Cytoscape software (version 3.8.2, http://www.cytoscape.org/) [29]. Subsequently, the topological properties including node degree [30], betweenness centrality [31], stress centrality [32] and closeness centrality [33] of the DEGs in the PPI network were calculated by Network Analyzer plug-in of Cytoscape to further analyze the candidate hub genes from the PPI network. The PEWCC1 plug-in [34] was used to search sub-networks of the PPI network and the default parameters (Degree cutoff $\geq 10$, node score cutoff $\geq 0.4$, K-core $\geq 4$, and $\max \operatorname{depth}=100$.) were set in the functional interface of Cytoscape software.

\section{MiRNA-hub gene regulatory network construction}

The miRNA-hub gene interactions were predicted with miRNet database (https://www.mirnet.ca/) [35], which involves 14 predicted algorithms (TarBase, miRTarBase, miRecords, miRanda (S mansoni only), miR2Disease, HMDD, PhenomiR, SM2miR, PharmacomiR, EpimiR, starBase, TransmiR, ADmiRE, and TAM 2.0). The Cytoscape 3.8.2 software [29] was used to visualize the regulatory network.

\section{TF-hub gene regulatory network construction}

The TF-hub gene interactions were predicted with NetworkAnalyst database (https://www.networkanalyst.ca/) [36], which involves 1 predicted algorithm (ChEA). The Cytoscape 3.8.2 software [29] was used to visualize the regulatory network.

\section{Receiver operating characteristic curve (ROC) analysis}

pROC package in $\mathrm{R}$ statistical software [37] was used to operate ROC curve analysis and to resolve the specificity, sensitivity, positive and negative predictive values for all the feasible thresholds of the ROC curve. The value of the hub genes were predicted based on the ROC curve analysis.

\section{Results}




\section{Identification of DEGs}

DEGs were identified by DESeq2 in the R package. In total, 953 DEGs (478 up and 475 down regulated genes) were selected based on the criteria of adj. $P$ value $<0.05,|\log 2(\mathrm{FC})|>1.73$ for up regulated genes and $|\log 2(\mathrm{FC})|>-0.69$ for down regulated genes. Heatmap of DEGs is presented in Fig. 1, and volcano plot is presented in Fig. 2. The up and down regulated DEGs is listed in Table 1.

\section{Gene Ontology (GO) and REACTOME pathway enrichment analysis of DEGs}

All 953 DEGs were analyzed by g:Profiler software and the results of GO enrichment analysis indicated that 1) for BP, up regulated genes were particularly enriched in multicellular organismal process and developmental process, and down regulated genes in cell activation and immune system process; 2) for $\mathrm{CC}$, up regulated genes were enriched in cell periphery and intrinsic component of membrane, and down regulated genes in endomembrane system and cytoplasm; 3) for GO MF, up regulated genes were significantly enriched in the transporter activity and channel activity, and down regulated geness in enzyme binding and protein binding (Table 2). REACTOME pathway enrichment analysis results were shown in Table 3 which demonstrated that up regulated genes were particularly enriched in formation of the cornified envelope and cell-cell junction organization, while down regulated genes were particularly enriched in hemostasis and neutrophil degranulation ( $\mathrm{P} \square<\square 0.05$ ).

\section{Construction of the PPI network and module analysis}

The PPI network of DEGs was constructed with 8472 nodes and 20624 edges (Fig.3). The node degree, betweenness centrality, stress centrality and closeness centrality methods were performed to calculate the top hub genes and are listed in Table 4. The results revealed 5 up and 5 down regulated genes identified as hub genes, including TRIM54, ELAVL2, PTN, KIT, BMPR1B, APP, SRC, ITGA4, RPA1 and ACTB. PEWCC1 was used to identify the significant modules in the PPI network and the two significant modules were selected. Module 1 consisted of 95 nodes and 103 edges (Fig.4A) and module 2 consisted of 79 nodes and 131 edges (Fig.4B). Following GO and pathway enrichment analysis, the module 1 was revealed to be associated with multicellular organismal process, developmental 
process and intrinsic component of membrane, and module 2 was revealed to be associated with hemostasis, immune system, enzyme binding, cytokine signaling in immune system and protein binding.

\section{MiRNA-hub gene regulatory network construction}

To investigate interaction correlations between the hub genes and miRNAs, a miRNA-hub gene interaction network was constructed, which has 2598 nodes (hub genes:307; miRNAs: 2291) and 17035 edges. Results from the current investigation demonstrated that one target gene might be regulated by several miRNAs (Fig. 5) and are listed in Table 5. Up regulated hub genes including, ERRFI1 targeted by 70 miRNAs (ex; hsa-mir-198); CCNE1 targeted by 50 miRNAs (ex; hsa-mir-181b-5p); ELAVL2 targeted by 43 miRNAs (ex; hsa-mir766-3p); ANTXR1 targeted by 36 miRNAs (ex; hsa-mir-624-5p); ZBTB8A targeted by 34 miRNAs (ex; hsa-mir-10b-5p). Similarly, down regulated hub genes including, SRRM2 targeted by 250 miRNAs (ex; hsa-mir-582-5p); CDKN1A targeted by 187 miRNAs (ex; hsa-mir-657); MYH9 targeted by 126 miRNAs (ex; hsa-mir-1231); APP targeted by 125 miRNAs (ex; hsa-mir-509-3-5p); ACTB targeted by 122 miRNAs (ex; hsa-mir-192-3p).

\section{TF-hub gene regulatory network construction}

To investigate interaction correlations between the hub genes and TFs, a TF-hub gene interaction network was constructed, which has 503 nodes (hub genes:309; miRNAs: 194) and 7686 edges. Results from the current investigation demonstrated that one target gene might be regulated by several TFs, (Fig. 6) and are listed in Table 5. Up regulated hub genes including, ERRFI1 targeted by 43 TFs (ex; CREM); ELAVL2 targeted by 41 TFs (ex; BMI1); KIT targeted by 34 TFs (ex; DMRT1); ZBTB8A targeted by 29 TFs (ex; GATA4); PPARGC1A targeted by 27 TFs (ex; FOXA2). Similarly, down regulated hub genes including, SMAD3 targeted by 58 TFs (ex; EP300); CDKN1A targeted by 57 TFs (ex; STAT3); ACTB targeted by 56 TFs (ex; HOXC9); PKM targeted by 55 TFs (ex; FOXP1); MYH9 targeted by 53 TFs (ex; ATF3).

\section{Receiver operating characteristic curve (ROC) analysis}


Based on the data of GSE154377, the patients were divided into the normal pregnancy group and GDM group. The ROC curves were analyzed by pROC package in $\mathrm{R}$ statistical software to evaluate the diagnostic accuracy of hub genes for GDM. As shown in Fig. 7, TRIM54, ELAVL2, PTN, KIT, BMPR1B, APP, SRC, ITGA4, RPA1 and ACTB achieved an AUC value of >0.90, demonstrating that these genes have high sensitivity and specificity for GDM diagnosis. Therefore, the ten hub genes identified, TRIM54, ELAVL2, PTN, KIT, BMPR1B, APP, SRC, ITGA4, RPA1 and ACTB might be considered as new biomarkers for GDM and require further investigation to validate these preliminary findings.

\section{Discussion}

A number of pre-clinical and clinical investigations have been conducted to reveal the underlying mechanisms of GDM in the past decades; however, the incidence of GDM remains high. This is primarily due to the majority of the investigation focusing genetic risk factor [38]. In the current investigation, expression profiling by high throughput sequencing dataset was analyzed and 953 DEGs (478 up and 475 down regulated genes) were identified. Existing evidences have reported that HNF4G [39], SLC15A1 [40], CXCL5 [41] and MALAT1 [42] promotes obesity, but these genes might be novel target for GDM. NPHP3-AS1 [43], LTA (lymphotoxin alpha) [44], CXCL5 [45] and MALAT1 [46] are found to be expressed in cardiovascular diseases, but these genes might be novel targets for GDM. A recent study demonstrated that XIST (X inactive specific transcript) [47] and MALAT1 [48] serves a crucial role in GDM progression. LTA (lymphotoxin alpha) [49], CXCL5 [50] and MALAT1 [51] are associated with prognosis of diabetes mellitus, but these genes might be novel targets for GDM. LTA (lymphotoxin alpha) [52], CXCL5 [53], MALAT1 [54] and TMEM70 [55] have been demonstrated to function in hypertension, but these genes might be novel targets for GDM. CXCL5 has been shown to be activated abortion [56].

GO and REACTOME pathway enrichment analyses were used to investigate the interactions of DEGs. Pathways include hemostasis [57], neutrophil degranulation [58], immune system [59] and cytokine signaling in immune system [60] are responsible for progression of GDM. LGR5 [61], GREM1 [62], GLRA3 [63], NEUROD4 [64], CYP2J2 [65], KCNH6 [66], LBP (lipopolysaccharide binding protein) [67], CXCL14 [68], RGN (regucalcin) [69], NPY2R [70], 
SERPINB13 [71], WNT5A [72], EDA (ectodysplasin A) [73], HSD11B2 [74], ACVR1C [75], NEUROD1 [76], SLIT2 [77], PPARGC1A [78], IGF1 [79], OSR1 [80], CYP46A1 [81], TLR3 [82], BMP7 [83], SELP (selectin P) [84], HLA-A [85], NOTCH2 [86], LRP1 [87], CLU (clusterin) [88], FCN1 [89], CDKN1A [90], SMAD3 [91], HLA-E [92], PTPRC (protein tyrosine phosphatase receptor type C) [93], MYH9 [94], JAK3 [95], IL6R [96], TIMP1 [97], DOCK8 [98], TNFRSF1B [99], ITGAL (integrin subunit alpha L) [100], CD47 [101], RARA (retinoic acid receptor alpha) [102], DGKD (diacylglycerol kinase delta) [103], PLEK (pleckstrin) [104], PREX1 [105], BSCL2 [106], PANX1 [107], IRF7 [108], NOTCH1 [109], STIM1 [110], TRIM13 [111], LRBA (LPS responsive beige-like anchor protein) [112], CXCR4 [113], MDM4 [114], MYO9B [115] and PDE5A [116] were revealed to be expressed in diabetes mellitus, but these genes might be novel targets for GDM. SIX1 [117], GREM1 [118], GHRHR (growth hormone releasing hormone receptor) [119], GPR37L1 [120], CYP2J2 [121], AQP4 [122], ROS1 [123], LBP (lipopolysaccharide binding protein) [124], SGCD (sarcoglycan delta) [125], CXCL14 [126], RGN (regucalcin) [127], F9 [128], KCND2 [129], AZGP1 [130], HAS2 [131], CNTN4 [132], WNT5A [133], PTHLH [134], FAP (fibroblast activation protein alpha) [135], THSD7A [136], SHROOM3 [137], ETV1 [138], CYP24A1 [139], SLIT2 [140], GJC1 [141], PPARGC1A [142], TRPM3 [143], IGF1 [144], TRPV6 [145], TLR3 [146], BMP7 [147], DSG2 [148], POSTN (periostin) [149], ENOX1 [150], KCNA7 [151], ITGB3 [152], THBS1 [153], TRPC6 [154], SELP (selectin P) [155], VCL (vinculin) [156], P2RY1 [157], NOTCH2 [158], F2RL3 [159], PSAP (prosaposin) [160], LRP1 [161], CLU (clusterin) [162], FCN1 [163], CDKN1A [164], SMAD3 [165], CAPN1 [166], BAK1 [167], FERMT3 [168], HLA-E [169], ADA2 [170], PTPRC (protein tyrosine phosphatase receptor type C) [171], PEAR1 [172], TGFB1 [173], JAK3 [174], ALOX12 [175], ITGB2 [176], SELPLG (selectin P ligand) [177], TNFSF4 [178], BCL11B [179], IL17RA [180], CST3 [181], BCL3 [182], TNFRSF1B [183], SERPINA1 [184], CD47 [185], CD74 [186], STXBP2 [187], LEF1 [188], F13A1 [189], BSCL2 [190], VCAN (versican) [191], SUN2 [192], PPM1L [193], IRF7 [194], NOTCH1 [195], EHD3 [196], PCSK6 [197], STIM1 [198], CXCR4 [199], ATP2A2 [200], CALM3 [201], CSF1R [202], CLPP (caseinolytic mitochondrial matrix peptidase proteolytic subunit) [203], PIK3IP1 [204], CDK9 [205], USP22 [206], MDM4 [207], TUBB1 [208], PDE5A [209] and GNB5 [210] plays important roles in cardiovascular diseases progression, but these genes might 
be novel targets for GDM. Polonikov et al [211], Chida et al [212], Yamada et al [213], Zhang et al [214], Kawarazaki et al [215], Ueda et al [216], Bao et al [217], Leng et al [218], Taghvaei et al [219], Norling et al [220], Brown et al [221], Singh et al [222], Liu et al [223], Nie et al [224], Liu et al [225], Sharma et al [226], Liu et al [227], Jain et al [228], Novoyatleva et al [229], Yu et al [230], Sahoo et al [231], Gamboa et al [232], Zeng et al [233], Zabini et al [234], Olivi et al [235], Liu et al [236], Nabrdalik et al [237], Katayose et al [238], Topchieva et al [239], Onal et al [240], Speirs et al [241], Novelli et al [242], Le Hiress et al [243], Chang et al [244], Deng et al [245], Wang et al [246], Dhande et al [247], Yu et al [248] and Li et al [249] showed that CYP2J2, BMPR1B, ROS1, SCN7A, WNT5A, HSD11B2, CYP24A1, WNK3, PPARGC1A, IGF1, OSR1, TLR3, BMP7, POSTN (periostin), ITGB3, THBS1, FLNA (filamin A), TRPC6, SELP (selectin P), VCL (vinculin), NOTCH2, F2RL3, LRP1, CLU (clusterin), SMAD3, PEAR1, MYH9, TGFB1, PDGFA (platelet derived growth factor subunit A), IL6R, TIMP1, TNFRSF1B, CD47, CD74, VCAN (versican), IRF7, NOTCH1, STIM1, CXCR4 and PDE5A expression can be associated with hypertension progression, but these genes might be novel targets for GDM. ROS1 [250], WNT2 [251], PHLDA2 [252], WNT5A [253], HSD11B2 [254], CLDN1 [255], CYP24A1 [256], HRG (histidine rich glycoprotein) [257], TLR3 [258], THBS1 [259], TRPC6 [260], SELP (selectin P) [261], CLU (clusterin) [262], CDKN1A [263], ITGB1 [264], HLA-E [265], IL6R [266], TIMP1 [267], SERPINA1 [268], CD74 [269], PANX1 [270], STIM1 [271] and CXCR4 [272] were revealed to be correlated with disease outcome in patients with preeclampsia. Previously reported studies have shown that the expression of LBP (lipopolysaccharide binding protein) [273], MC1R [274], CXCL14 [275], RGN (regucalcin) [276], NPY2R [277], MFAP5 [278], WNT5A [279], EDA (ectodysplasin A) [280], THSD7A [281], NEUROD1 [282], SLIT2 [283], PPARGC1A [219], IGF1 [144], OSR1 [284], TLR3 [285], BMP7 [286], POSTN (periostin) [287], LRP1B [288], THBS1 [289], NOTCH2 [290]. LRP1 [291], CLU (clusterin) [292], SMAD3 [91], TGFB1 [293], APP (amyloid beta precursor protein) [294], ITGB2 [295], IL6R [296], TIMP1 [297], CD47 [298], CD74 [299], RARA (retinoic acid receptor alpha) [300], DOCK2 [301], F13A1 [302], IRF7 [303], STIM1 [304], CXCR4 [305], MGLL (monoglyceride lipase) [306], M6PR [307], USP22 [308] and CASP2 [309] were mainly involved in progression of obesity, but these genes might be novel targets for GDM. Recent studies have shown that GLP1R [310], NEUROD1 [311], PPARGC1A [312], 
IGF1 [313], LRP1B [314], NOTCH2 [315], BAK1 [316], TLR2 [317], IL6R [318], TIMP1 [319], PIK3CD [320], PRKCA (protein kinase C alpha) [321], CXCR4 [322], RAB8A [323] and M6PR [324] are associated with GDM. Abnormal expression PDE5A was linked with abortion [325].

Hub genes in the PPI network and modules are linked to the progression of GDM. TRIM54, ELAVL2, PTN (pleiotrophin), KIT (KIT proto-oncogene, receptor tyrosine kinase), SRC (SRC proto-oncogene, non-receptor tyrosine kinase), ITGA4, RPA1, ACTB (actin beta) and IGSF1 were might be the novel biomarkers for GDM.

At the same time, hub genes, miRNAs and TFs imlpicated with GDM, all of which were located in the core nodes in MiRNA-hub gene regulatory network and TF-hub gene regulatory network, meaning that these hub genes, miRNAs and TFs could be critical therapeutic targets to protect against GDM. hsa-mir-766-3p [326], hsa-mir-10b-5p [327], hsa-mir-657 [328], GATA4 [329], FOXA2 [330], STAT3 [331] and ATF3 [332] plays an important role in the progression of diabetes mellitus, but these miRNAs might be novel targets for GDM. hsa-mir-582-5p is closely related to obesity [333], but these miRNAs might be novel targets for GDM. Wang et al. [334] found that the altered expression of hsa-mir-657 in GDM. Improta Caria et al [335], Li et al. [336], Park et al. [337], Cheng et al. [338] and Davis, [339] demonstrated that hsa-mir-657, BMI1, GATA4, STAT3 and ATF3 played a pivotal role in the hypertension, but these miRNAs might be novel targets for GDM. Improta Caria et al [335], Patankar et al. [340], Bochkis et al. [341], Nishimura et al. [342], Su et al. [343] and Kim et al. [344] suggested that hsa-mir-657, GATA4, FOXA2, EP300, STAT3 and ATF3 could induce obesity, but these genes might be novel targets for GDM. Accumulating evidence has demonstrated that hsa-mir-657 [345], hsa-mir-1231 [346], CREM (cAMP responsive element modulator) [347], BMI1 [348], GATA4 [349], EP300 [350], STAT3 [351], FOXP1 [352] and ATF3 [353] appears to be constitutively activated in cardiovascular diseases, but these genes might be novel targets for GDM. Abnormal expression of FOXP1 was responsible for loss of pregnancy [354]. ERRFI1, CCNE1, ANTXR1, ZBTB8A, SRRM2, PKM (pyruvate kinase M1/2), hsa-mir-198, hsa-mir-181b-5p, hsa-mir-624-5p, hsa-mir-509-3-5p, hsa-mir-1923p, DMRT1, HOXC9, were might be the novel biomarkers for GDM. 


\section{Conclusions}

The findings from this bioinformatics analysis investigations identified signaling pathwayes and the essential hub genes that might contribute to GDM. These findings improve our understanding of the molecular pathogenesis of GDM. Our investigation has key clinical importance for the early diagnosis and treatment, as well as the prevention, of GDM and provides effective targets for the treatment of GDM. However, further validation experiments are required to confirm the function of the identified hub genes linked with GDM.

\section{Acknowledgement}

I thank Giorgia Del Vecchio, University of California Los Angeles, Los Angeles, USA, very much, the author who deposited their profiling by high throughput sequencing dataset GSE154377, into the public GEO database.

\section{Conflict of interest}

The authors declare that they have no conflict of interest.

\section{Ethical approval}

This article does not contain any studies with human participants or animals performed by any of the authors.

\section{Informed consent}

No informed consent because this study does not contain human or animals participants.

\section{Availability of data and materials}

The datasets supporting the conclusions of this article are available in the GEO (Gene Expression Omnibus) (https://www.ncbi.nlm.nih.gov/geo/) repository. [(GSE154377) https://www.ncbi.nlm.nih.gov/geo/query/acc.cgi?acc=GSE154377)]

\section{Consent for publication}

Not applicable.

\section{Competing interests}


The authors declare that they have no competing interests.

\section{Author Contributions}

B. V. - Writing original draft, and review and editing

C. V. - Software and investigation

\section{Authors}

Basavaraj Vastrad

ORCID ID: 0000-0003-2202-7637

Chanabasayya Vastrad

ORCID ID: $\underline{0000-0003-3615-4450}$

\section{References}

1. Chiefari E, Arcidiacono B, Foti D, Brunetti A. Gestational diabetes mellitus: an updated overview. J Endocrinol Invest. 2017;40(9):899-909. doi:10.1007/s40618-016-0607-5

2. Lende M, Rijhsinghani A. Gestational Diabetes: Overview with Emphasis on Medical Management. Int $\mathrm{J}$ Environ Res Public Health. 2020;17(24):9573.. doi:10.3390/ijerph17249573

3. Brown J, Ceysens G, Boulvain M. Exercise for pregnant women with gestational diabetes for improving maternal and fetal outcomes. Cochrane Database Syst Rev. 2017;6(6):CD012202. doi:10.1002/14651858.CD012202.pub2

4. Moon JH, Kwak SH, Jang HC. Prevention of type 2 diabetes mellitus in women with previous gestational diabetes mellitus. Korean J Intern Med. 2017;32(1):26-41. doi:10.3904/kjim.2016.203

5. Callaway LK, McIntyre HD, Barrett HL, Foxcroft K, Tremellen A, Lingwood BE, Tobin JM, Wilkinson S, Kothari A, Morrison M, et al. Probiotics for the Prevention of Gestational Diabetes Mellitus in Overweight and Obese Women: Findings From the SPRING Double-Blind Randomized Controlled Trial. Diabetes Care. 2019;42(3):364-371. doi:10.2337/dc182248

6. Wang JW, Cao SS, Hu RY, Wang M. Association between cigarette smoking during pregnancy and gestational diabetes mellitus: a meta- 
analysis. J Matern Fetal Neonatal Med. 2020;33(5):758-767. doi:10.1080/14767058.2018.1500547

7. Dalfrà MG, Burlina S, Del Vescovo GG, Lapolla A. Genetics and Epigenetics: New Insight on Gestational Diabetes Mellitus. Front Endocrinol (Lausanne). 2020;11:602477. doi:10.3389/fendo.2020.602477

8. Daly B, Toulis KA, Thomas N, Gokhale K, Martin J, Webber J, Keerthy D, Jolly K, Saravanan P, Nirantharakumar K. Increased risk of ischemic heart disease, hypertension, and type 2 diabetes in women with previous gestational diabetes mellitus, a target group in general practice for preventive interventions: A population-based cohort study. PLoS Med. 2018;15(1):e1002488. doi:10.1371/journal.pmed.1002488

9. Lorenzo-Almorós A, Hang T, Peiró C, Soriano-Guillén L, Egido J, Tuñón J, Lorenzo Ó. Predictive and diagnostic biomarkers for gestational diabetes and its associated metabolic and cardiovascular diseases. Cardiovasc Diabetol. 2019;18(1):140. doi:10.1186/s12933-019-0935-9

10.Wang Q, Lu X, Li C, Zhang W, Lv Y, Wang L, Wu L, Meng L, Fan Y, Ding $\mathrm{H}$, et al. Down-regulated long non-coding RNA PVT1 contributes to gestational diabetes mellitus and preeclampsia via regulation of human trophoblast cells. Biomed Pharmacother. 2019;120:109501. doi:10.1016/j.biopha.2019.109501

11. Wei Q, Chen X, Chen H. Association of Single Nucleotide Polymorphisms of the IL-6, IL-10, and TNF- $\alpha$ Genes with Susceptibility to Gestational Diabetes Mellitus. Genet Test Mol Biomarkers. 2020;24(7):390-398. doi:10.1089/gtmb.2020.0069

12.Zhang X, Shi C, Wei L, Sun F, Ji L. The Association Between the rs2975760 and rs3792267 Single Nucleotide Polymorphisms of Calpain 10 (CAPN10) and Gestational Diabetes Mellitus. Med Sci Monit. 2019;25:5137-5142. doi:10.12659/MSM.914930

13.Li X, Yang W, Fang Y. Epigenetic Profiles Reveal That ADCYAP1 Serves as Key Molecule in Gestational Diabetes Mellitus. Comput Math Methods Med. 2019;2019:6936175. doi:10.1155/2019/6936175

14.Peng HY, Li HP, Li MQ. Downregulated ABHD5 Aggravates Insulin Resistance of Trophoblast Cells During Gestational Diabetes Mellitus. Reprod Sci. 2020;27(1):233-245. doi:10.1007/s43032-019-00010-х 
15.Chen SH, Liu XN, Peng Y. MicroRNA-351 eases insulin resistance and liver gluconeogenesis via the PI3K/AKT pathway by inhibiting FLOT2 in mice of gestational diabetes mellitus. J Cell Mol Med. 2019;23(9):58955906. doi:10.1111/jcmm.14079

16.Zhang Z, Zhao H, Wang A. Oleuropein alleviates gestational diabetes mellitus by activating AMPK signaling. Endocr Connect. 2021;10(1):45-53. doi:10.1530/EC-20-0466

17.Shi A, Wen J, Liu G, Liu H, Fu Z, Zhou J, Zhu Y, Liu Y, Guo X, Xu J. Genetic variants in vitamin $\mathrm{D}$ signaling pathways and risk of gestational diabetes mellitus. Oncotarget. 2016;7(42):67788-67795. doi:10.18632/oncotarget.11984

18.Liu T, Zheng W, Wang L, Wang L, Zhang Y. TLR4/NF- $\kappa$ B Signaling Pathway Participates in the Protective Effects of Apocynin on Gestational Diabetes Mellitus Induced Placental Oxidative Stress and Inflammation. Reprod Sci. 2020;27(2):722-730. doi:10.1007/s43032-019-00078-5

19.Dai S, Meng X, Cai X, Yuan C, Zhao Z, Zhong L, Shi Y, Yin F. Therapeutic effect of ursolic acid on fetal development in pregnant rats with gestational diabetes mellitus via AGEs-RAGE signaling pathway. J Food Biochem. 2021;45(4):e13651. doi:10.1111/jfbc. 13651

20.Stenson PD, Mort M, Ball EV, Evans K, Hayden M, Heywood S, Hussain M, Phillips AD, Cooper DN. The Human Gene Mutation Database: towards a comprehensive repository of inherited mutation data for medical research, genetic diagnosis and next-generation sequencing studies. Hum Genet. 2017;136(6):665-677. doi:10.1007/s00439-017-1779-6

21.Clough E, Barrett T. The Gene Expression Omnibus Database. Methods Mol Biol. 2016;1418:93-110. doi:10.1007/978-1-4939-3578-9_5

22. Yan L, Feng J, Cheng F, Cui X, Gao L, Chen Y, Wang F, Zhong T, Li Y, Liu L. Circular RNA expression profiles in placental villi from women with gestational diabetes mellitus. Biochem Biophys Res Commun. 2018;498(4):743-750. doi:10.1016/j.bbrc.2018.03.051

23.Del Vecchio G, Li Q, Li W, Thamotharan S, Tosevska A, Morselli M, Sung K, Janzen C, Zhou X, Pellegrini M, et al. Cell-free DNA Methylation and Transcriptomic Signature Prediction of Pregnancies with Adverse Outcomes. $\quad$ Epigenetics. 2021;16(6):642-661. doi:10.1080/15592294.2020.1816774 
24.Love MI, Huber W, Anders S. Moderated estimation of fold change and dispersion for RNA-seq data with DESeq2. Genome Biol. 2014;15(12):550. doi:10.1186/s13059-014-0550-8

25.Thomas PD. The Gene Ontology and the Meaning of Biological Function.

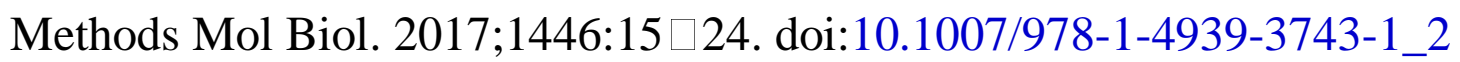

26.Fabregat A, Jupe S, Matthews L, Sidiropoulos K, Gillespie M, Garapati P, Haw R, Jassal B, Korninger F, May B et al The Reactome Pathway Knowledgebase. Nucleic Acids Res. 2018;46(D1):D649-D655. doi:10.1093/nar/gkx1132

27.Reimand J, Kull M, Peterson H, Hansen J, Vilo J. g:Profiler--a web-based toolset for functional profiling of gene lists from large-scale experiments. Nucleic Acids Res. 2007;35(Web Server issue):W193-W200. doi:10.1093/nar/gkm226

28.Pastrello C, Kotlyar M, Jurisica I. Informed Use of Protein-Protein Interaction Data: A Focus on the Integrated Interactions Database (IID). Methods Mol Biol. 2020;2074:125-134. doi:10.1007/978-1-4939-9873-9_10

29. Shannon P, Markiel A, Ozier O, Baliga NS, Wang JT, Ramage D, Amin N, Schwikowski B, Ideker T Cytoscape: a software environment for integrated models of biomolecular interaction networks. Genome Res 2003;13(11):2498-2504. doi:10.1101/gr.1239303

30.Przulj N, Wigle DA, Jurisica I. Functional topology in a network of protein interactions. Bioinformatics. 2004;20(3):340-348. doi:10.1093/bioinformatics/btg415

31.Nguyen TP, Liu WC, Jordán F. Inferring pleiotropy by network analysis: linked diseases in the human PPI network. BMC Syst Biol. 2011;5:179. doi:10.1186/1752-0509-5-179

32. Shi Z, Zhang B. Fast network centrality analysis using GPUs. BMC Bioinformatics. 2011;12:149. doi:10.1186/1471-2105-12-149

33.Fadhal E, Gamieldien J, Mwambene EC. Protein interaction networks as metric spaces: a novel perspective on distribution of hubs. BMC Syst Biol. 2014;8:6. doi:10.1186/1752-0509-8-6

34.Zaki N, Efimov D, Berengueres J. Protein complex detection using interaction reliability assessment and weighted clustering coefficient. BMC Bioinformatics. 2013;14:163. doi:10.1186/1471-2105-14 
35.Fan Y, Xia J (2018) miRNet-Functional Analysis and Visual Exploration of miRNA-Target Interactions in a Network Context. Methods Mol Biol 1819:215-233. doi:10.1007/978-1-4939-8618-7_10

36.Zhou G, Soufan O, Ewald J, Hancock REW, Basu N, Xia J (2019) NetworkAnalyst 3.0: a visual analytics platform for comprehensive gene expression profiling and meta-analysis. Nucleic Acids Res 47:W234-W241. doi:10.1093/nar/gkz240

37.Robin X, Turck N, Hainard A, Tiberti N, Lisacek F, Sanchez JC, Müller M. pROC: an open-source package for $\mathrm{R}$ and $\mathrm{S}+$ to analyze and compare ROC curves. BMC Bioinformatics 2011;12:77. doi:10.1186/1471-2105-12-77

38. Rosik J, Szostak B, Machaj F, Pawlik A. The role of genetics and epigenetics in the pathogenesis of gestational diabetes mellitus. Ann Hum Genet. 2020;84(2):114-124. doi:10.1111/ahg.12356

39.Ayari S, Gil-Iturbe E, le Gléau L, Osinski C, Kapel N, Soula HA, Leturque A, Andreelli F, Clément K, Serradas P, et al. HNF4g invalidation prevents diet-induced obesity via intestinal lipid malabsorption. J Endocrinol. 2021;JOE-21-0092.R1. doi:10.1530/JOE-21-0092

40.Wang CY, Liu S, Xie XN, Luo ZY, Yang L, Tan ZR. Association between polymorphisms in SLC15A1 and PLA2G16 genes and development of obesity in Chinese subjects. Diabetes Metab Syndr Obes. 2018;11:439-446. doi:10.2147/DMSO.S161808

41.Sepuru KM, Poluri KM, Rajarathnam K. Solution structure of CXCL5--a novel chemokine and adipokine implicated in inflammation and obesity. PLoS One. 2014;9(4):e93228. doi:10.1371/journal.pone.0093228

42.Ebrahimi R, Toolabi K, Jannat Ali Pour N, Mohassel Azadi S, Bahiraee A, Zamani-Garmsiri F, Emamgholipour S. Adipose tissue gene expression of long non-coding RNAs; MALAT1, TUG1 in obesity: is it associated with metabolic profile and lipid homeostasis-related genes expression?. Diabetol Metab Syndr. 2020;12:36. doi:10.1186/s13098-020-00544-0

43.Zhao Z, Liu G, Zhang H, Ruan P, Ge J, Liu Q. BIRC5, GAJ5, and lncRNA NPHP3-AS1 Are Correlated with the Development of Atrial FibrillationValvular Heart Disease. Int Heart J. 2021;62(1):153-161. doi:10.1536/ihj.20238

44.Palikhe A, Lokki ML, Pussinen PJ, Paju S, Ahlberg J, Asikainen S, Seppänen M, Valtonen V, Nieminen MS, Sinisalo J. Lymphotoxin alpha 
LTA+496C allele is a risk factor for periodontitis in patients with coronary artery disease. Tissue Antigens. 2008;71(6):530-537. doi:10.1111/j.13990039.2008.01038.x

45.Ravi S, Schuck RN, Hilliard E, Lee CR, Dai X, Lenhart K, Willis MS, Jensen BC, Stouffer GA, Patterson C, et al. Clinical Evidence Supports a Protective Role for CXCL5 in Coronary Artery Disease. Am J Pathol. 2017;187(12):2895-2911. doi:10.1016/j.ajpath.2017.08.006

46. Yan Y, Song D, Song X, Song C. The role of lncRNA MALAT1 in cardiovascular disease. IUBMB Life. 2020;72(3):334-342. doi:10.1002/iub.2210

47.Li Y, Yuan X, Shi Z, Wang H, Ren D, Zhang Y, Fan Y, Liu Y, Cui Z. LncRNA XIST serves as a diagnostic biomarker in gestational diabetes mellitus and its regulatory effect on trophoblast cell via miR-497-5p/FOXO1 axis. Cardiovasc Diagn Ther. 2021;11(3):716-725. doi:10.21037/cdt-21-110

48.Zhang Y, Qu L, Ni H, Wang Y, Li L, Yang X, Wang X, Hou Y. Expression and function of lncRNA MALAT1 in gestational diabetes mellitus. Adv Clin Exp Med. 2020;29(8):903-910. doi:10.17219/acem/121524

49.Kaidonis G, Craig JE, Gillies MC, Abhary S, Essex RW, Chang JH, Pal B, Pefkianaki M, Daniell M, Lake S, et al. Promoter polymorphism at the tumour necrosis factor/lymphotoxin-alpha locus is associated with type of diabetes but not with susceptibility to sight-threatening diabetic retinopathy. Diab Vasc Dis Res. 2016;13(2):164-167. doi:10.1177/1479164115616902

50.Hasani Ranjbar S, Amiri P, Zineh I, Langaee TY, Namakchian M, Heshmet R, Sajadi M, Mirzaee M, Rezazadeh E, Balaei P, et al. CXCL5 gene polymorphism association with diabetes mellitus. Mol Diagn Ther. 2008;12(6):391-394. doi:10.1007/BF03256304

51.Alfaifi M, Ali Beg MM, Alshahrani MY, Ahmad I, Alkhathami AG, Joshi PC, Alshehri OM, Alamri AM, Verma AK. Circulating long non-coding RNAs NKILA, NEAT1, MALAT1, and MIAT expression and their association in type 2 diabetes mellitus. BMJ Open Diabetes Res Care. 2021;9(1):e001821. doi:10.1136/bmjdrc-2020-001821

52.Nakayama T, Soma M, Sato N, Haketa A, Kosuge K, Aoi N, Sato M, Izumi Y, Matsumoto K, Kanmatsuse K, et al. An association study in essential hypertension using functional polymorphisms in lymphotoxin-alpha gene. 
Am J Hypertens. 2004;17(11 Pt 1):1045-1049. doi:10.1016/j.amjhyper.2004.07.010

53.Beitelshees AL, Aquilante CL, Allayee H, Langaee TY, Welder GJ, Schofield RS, Zineh I. CXCL5 polymorphisms are associated with variable blood pressure in cardiovascular disease-free adults. Hum Genomics. 2012;6(1):9. doi:10.1186/1479-7364-6-9

54.Ou M, Zhao H, Ji G, Zhao X, Zhang Q. Long noncoding RNA MALAT1 contributes to pregnancy-induced hypertension development by enhancing oxidative stress and inflammation through the regulation of the miR-1505p/ET-1 axis. FASEB J. 2020;34(5):6070-6085. doi:10.1096/fj.201902280R 55.Catteruccia M, Verrigni D, Martinelli D, Torraco A, Agovino T, Bonafé L, D'Amico A, Donati MA, Adorisio R, Santorelli FM, et al. Persistent pulmonary arterial hypertension in the newborn (PPHN): a frequent manifestation of TMEM70 defective patients. Mol Genet Metab. 2014;111(3):353-359. doi:10.1016/j.ymgme.2014.01.001

56.Zhang S, Ding J, Wang J, Yin T, Zhang Y, Yang J. CXCL5 Downregulation in Villous Tissue Is Correlated With Recurrent Spontaneous Abortion. Front Immunol. 2021;12:717483. doi:10.3389/fimmu.2021.717483

57.Abdel Gader AG, Khashoggi TY, Habib F, Awadallah SB. Haemostatic and cytokine changes in gestational diabetes mellitus. Gynecol Endocrinol. 2011;27(5):356-360. doi:10.3109/09513590.2010.495241

58.Shen D, Lu Y, Li G, Hu M, Li S, Ju H, Zhang M, Wang X. Mechanism of neutrophil extracellular traps generation and their role in trophoblast apoptosis in gestational diabetes mellitus. Cell Signal. 2021;110168. doi:10.1016/j.cellsig.2021.110168

59.Lin HC, Su CT, Wang PC. An application of artificial immune recognition system for prediction of diabetes following gestational diabetes. J Med Syst. 2011;35(3):283-289. doi:10.1007/s10916-009-9364-8

60.Kuzmicki M, Telejko B, Wawrusiewicz-Kurylonek N, Citko A, Lipinska D, Pliszka J, Wilk J, Kalejta K, Lemancewicz A, Grabiec M, et al. The expression of suppressor of cytokine signaling 1 and 3 in fat and placental tissue from women with gestational diabetes. Gynecol Endocrinol. 2012;28(11):841-844. doi:10.3109/09513590.2012.683055

61.Alharbi KK, Ali Khan I, Syed R, Alharbi FK, Mohammed AK, Vinodson B, Al-Daghri NM. Association of JAZF1 and TSPAN8/LGR5 variants in 
relation to type 2 diabetes mellitus in a Saudi population. Diabetol Metab Syndr. 2015;7:92. doi:10.1186/s13098-015-0091-7

62.Thameem F, Puppala S, He X, Arar NH, Stern MP, Blangero J, Duggirala R, Abboud HE. Evaluation of gremlin 1 (GREM1) as a candidate susceptibility gene for albuminuria-related traits in Mexican Americans with type 2 diabetes mellitus. Metabolism. 2009;58(10):1496-1502. doi:10.1016/j.metabol.2009.04.039

63.Sandholm N, Haukka JK, Toppila I, Valo E, Harjutsalo V, Forsblom C, Groop PH. Confirmation of GLRA3 as a susceptibility locus for albuminuria in Finnish patients with type 1 diabetes. Sci Rep. 2018;8(1):12408. doi:10.1038/s41598-018-29211-1

64. Horikawa Y, Horikawa Y, Cox NJ, Iwasaki N, Ogata M, Iwamoto Y, Schwitzgebel V, German MS, Bell GI. beta-cell transcription factors and diabetes: no evidence for diabetes-associated mutations in the gene encoding the basic helix-loop-helix transcription factor neurogenic differentiation 4 (NEUROD4) in Japanese patients with MODY. Diabetes. 2000;49(11):1955-1957. doi:10.2337/diabetes.49.11.1955

65.Wang CP, Hung WC, Yu TH, Chiu CA, Lu LF, Chung FM, Hung CH, Shin SJ, Chen HJ, Lee YJ. Genetic variation in the G-50T polymorphism of the cytochrome P450 epoxygenase CYP2J2 gene and the risk of younger onset type 2 diabetes among Chinese population: potential interaction with body mass index and family history. Exp Clin Endocrinol Diabetes. 2010;118(6):346-352. doi:10.1055/s-0029-1243604

66. Yang JK, Lu J, Yuan SS, Asan, Cao X, Qiu HY, Shi TT, Yang FY, Li Q, Liu CP, et al. From Hyper- to Hypoinsulinemia and Diabetes: Effect of KCNH6 on Insulin Secretion. Cell Rep. 2018;25(13):3800-3810.e6. doi:10.1016/j.celrep.2018.12.005

67.Sakura T, Morioka T, Shioi A, Kakutani Y, Miki Y, Yamazaki Y, Motoyama K, Mori K, Fukumoto S, Shoji T, et al. Lipopolysaccharidebinding protein is associated with arterial stiffness in patients with type 2 diabetes: a cross-sectional study. Cardiovasc Diabetol. 2017;16(1):62. doi:10.1186/s12933-017-0545-3

68. Matsushita Y, Hasegawa Y, Takebe N, Onodera K, Shozushima M, Oda T, Nagasawa K, Honma H, Nata K, Sasaki A, et al. Serum C-X-C motif 
chemokine ligand 14 levels are associated with serum C-peptide and fatty liver index in type 2 diabetes mellitus patients. J Diabetes Investig. 2021;12(6):1042-1049. doi:10.1111/jdi.13438

69. Yamaguchi M, Murata T. Involvement of regucalcin in lipid metabolism and diabetes. Metabolism. 2013;62(8):1045-1051. doi:10.1016/j.metabol.2013.01.023

70.Campbell CD, Lyon HN, Nemesh J, Drake JA, Tuomi T, Gaudet D, Zhu X, Cooper RS, Ardlie KG, Groop LC, et al. Association studies of BMI and type 2 diabetes in the neuropeptide Y pathway: a possible role for NPY2R as a candidate gene for type 2 diabetes in men. Diabetes. 2007;56(5):14601467. doi:10.2337/db06-1051

71.Kryvalap Y, Jiang ML, Kryvalap N, Hendrickson C, Czyzyk J. SerpinB13 antibodies promote $\beta$ cell development and resistance to type 1 diabetes. Sci Transl Med. 2021;13(588):eabf1587. doi:10.1126/scitranslmed.abf1587

72.Bretón-Romero R, Feng B, Holbrook M, Farb MG, Fetterman JL, Linder EA, Berk BD, Masaki N, Weisbrod RM, Inagaki E, et al. Endothelial Dysfunction in Human Diabetes Is Mediated by Wnt5a-JNK Signaling. Arterioscler Thromb Vasc Biol. 2016;36(3):561-569. doi:10.1161/ATVBAHA.115.306578

73.Bayliss J, Ooi GJ, De Nardo W, Shah YJH, Montgomery MK, McLean C, Kemp W, Roberts SK, Brown WA, Burton PR, et al. Ectodysplasin A Is Increased in Non-Alcoholic Fatty Liver Disease, But Is Not Associated With Type 2 Diabetes. Front Endocrinol (Lausanne). 2021;12:642432. doi:10.3389/fendo.2021.642432

74. Mune T, Suwa T, Morita H, Isomura Y, Takada N, Yamamoto Y, Hayashi M, Yamakita N, Sasaki A, Takeda N, et al. Longer HSD11B2 CA-repeat in impaired glucose tolerance and type 2 diabetes. Endocr J. 2013;60(5):671678. doi:10.1507/endocrj.ej12-0108

75.Emdin CA, Khera AV, Aragam K, Haas M, Chaffin M, Klarin D, Natarajan P, Bick A, Zekavat SM, Nomura A, et al. DNA Sequence Variation in ACVR1C Encoding the Activin Receptor-Like Kinase 7 Influences Body Fat Distribution and Protects Against Type 2 Diabetes. Diabetes. 2019;68(1):226-234. doi:10.2337/db18-0857

76.Demirbilek H, Hatipoglu N, Gul U, Tatli ZU, Ellard S, Flanagan SE, De Franco E, Kurtoglu S. Permanent neonatal diabetes mellitus and 
neurological abnormalities due to a novel homozygous missense mutation in NEUROD1. Pediatr Diabetes. 2018;19(5):898-904. doi:10.1111/pedi.12669

77.Kang YE, Choung S, Lee JH, Kim HJ, Ku BJ. The Role of Circulating Slit2, the One of the Newly Batokines, in Human Diabetes Mellitus. Endocrinol Metab (Seoul). 2017;32(3):383-388. doi:10.3803/EnM.2017.32.3.383

78.Zhu L, Huang Q, Xie Z, Kang M, Ding H, Chen B, Chen Y, Liu C, Wang Y, Tang W. PPARGC1A rs3736265 G>A polymorphism is associated with decreased risk of type 2 diabetes mellitus and fasting plasma glucose level. Oncotarget. 2017;8(23):37308-37320. doi:10.18632/oncotarget.16307

79.van Dijk PR, Logtenberg SJ, Groenier KH, Kleefstra N, Bilo HJ, Arnqvist HJ. Effect of i.p. insulin administration on IGF1 and IGFBP1 in type 1 diabetes. Endocr Connect. 2014;3(1):17-23. doi:10.1530/EC-13-0089

80.Guo Q, Zhang Y, Jiang GR, Zhang C. Decreased KLHL3 expression is involved in the activation of WNK-OSR1/SPAK-NCC cascade in type 1 diabetic mice. Pflugers Arch. 2021;473(2):185-196. doi:10.1007/s00424020-02509-8

81.Shi J, Jia J, Tian S, Zhang H, An K, Zhu W, Cao W, Yuan Y, Wang S. Increased Plasma Level of 24S-Hydroxycholesterol and Polymorphism of CYP46A1 SNP (rs754203) Are Associated With Mild Cognitive Impairment in Patients With Type 2 Diabetes. Front Aging Neurosci. 2021;13:619916. doi:10.3389/fnagi.2021.619916

82.Zhou Z, Zeng C, Nie L, Huang S, Guo C, Xiao D, Han Y, Ye X, Ou M, Huang $\mathrm{C}$, et al. The effects of TLR3, TRIF and TRAF3 SNPs and interactions with environmental factors on type 2 diabetes mellitus and vascular complications in a Han Chinese population. Gene. 2017;626:41-47. doi:10.1016/j.gene.2017.05.011

83. Chattopadhyay T, Singh RR, Gupta S, Surolia A. Bone morphogenetic protein-7 (BMP-7) augments insulin sensitivity in mice with type II diabetes mellitus by potentiating PI3K/AKT pathway. Biofactors. 2017;43(2):195209. doi:10.1002/biof.1334

84.Kaur R, Singh J, Kapoor R, Kaur M. Association of SELP Polymorphisms with Soluble P-Selectin Levels and Vascular Risk in Patients with Type 2 Diabetes Mellitus: A Case-Control Study. Biochem Genet. 2019;57(1):7397. doi:10.1007/s10528-018-9881-6 
85.Howson JM, Walker NM, Clayton D, Todd JA; Type 1 Diabetes Genetics Consortium. Confirmation of HLA class II independent type 1 diabetes associations in the major histocompatibility complex including HLA-B and HLA-A. Diabetes Obes Metab. 2009;11 Suppl 1(Suppl 1):31-45. doi:10.1111/j.1463-1326.2008.01001.x

86.Al-Awaida WJ, Hameed WS, Al Hassany HJ, Al-Dabet MM, Al-Bawareed O, Hadi NR. Evaluation of the Genetic Association and Expressions of Notch-2 /Jagged-1 in Patients with Type 2 Diabetes Mellitus. Med Arch. 2021;75(2):101-108. doi:10.5455/medarh.2021.75.101-108

87.El-Horany HE, Watany MM, Hagag RY, El-Attar SH, Basiouny MA. Expression of LRP1 and CHOP genes associated with peripheral neuropathy in type 2 diabetes mellitus: Correlations with nerve conduction studies. Gene. 2019;702:114-122. doi:10.1016/j.gene.2019.02.105

88. Cai R, Han J, Sun J, Huang R, Tian S, Shen Y, Dong X, Xia W, Wang S. Plasma Clusterin and the CLU Gene rs11136000 Variant Are Associated with Mild Cognitive Impairment in Type 2 Diabetic Patients. Front Aging Neurosci. 2016;8:179. doi:10.3389/fnagi.2016.00179

89. Anjosa ZP, Santos MM, Rodrigues NJ, Lacerda GA, Araujo J, Silva JA, Tavares NA, Guimarães RL, Crovella S, Brandão LA. Polymorphism in ficolin-1 (FCN1) gene is associated with an earlier onset of type 1 diabetes mellitus in children and adolescents from northeast Brazil. J Genet. 2016;95(4):1031-1034. doi:10.1007/s12041-016-0719-x

90.Muhammad SA, Qousain Naqvi ST, Nguyen T, Wu X, Munir F, Jamshed $\mathrm{MB}$, Zhang Q. Cisplatin's potential for type 2 diabetes repositioning by inhibiting CDKN1A, FAS, and SESN1. Comput Biol Med. 2021;135:104640. doi:10.1016/j.compbiomed.2021.104640

91. Yadav H, Quijano C, Kamaraju AK, Gavrilova O, Malek R, Chen W, Zerfas $\mathrm{P}$, Zhigang D, Wright EC, Stuelten C, et al. Protection from obesity and diabetes by blockade of TGF- $\beta / \mathrm{Smad} 3$ signaling. Cell Metab. 2011;14(1):67-79. doi:10.1016/j.cmet.2011.04.013

92.Jiang H, Canfield SM, Gallagher MP, Jiang HH, Jiang Y, Zheng Z, Chess L. HLA-E-restricted regulatory CD8(+) T cells are involved in development and control of human autoimmune type 1 diabetes. J Clin Invest. 2010;120(10):3641-3650. doi:10.1172/JCI43522 
93.Thude H, Rosenhahn S, Hunger-Dathe W, Müller UA, Barz D. A transmembrane protein-tyrosine phosphatase receptor type $\mathrm{C}$ (CD45) exon A point mutation ( $77 \mathrm{C}$ to $\mathrm{G}$ ) is not associated with the development of type 1 diabetes mellitus in a German population. Eur $\mathrm{J}$ Immunogenet. 2004;31(6):245-247. doi:10.1111/j.1365-2370.2004.00479.x

94.Freedman BI, Langefeld CD, Lu L, Divers J, Comeau ME, Kopp JB, Winkler CA, Nelson GW, Johnson RC, Palmer ND, et al. Differential effects of MYH9 and APOL1 risk variants on FRMD3 Association with Diabetic ESRD in African Americans. PLoS Genet. 2011;7(6):e1002150. doi:10.1371/journal.pgen.1002150

95.Cetkovic-Cvrlje M, Dragt AL, Vassilev A, Liu XP, Uckun FM. Targeting JAK3 with JANEX-1 for prevention of autoimmune type 1 diabetes in NOD mice. Clin Immunol. 2003;106(3):213-225. doi:10.1016/s15216616(02)00049-9

96.Wu X, Yu T, Ji N, Huang Y, Gao L, Shi W, Yan Y, Li H, Ma L, Wu K, Wu Z. IL6R inhibits viability and apoptosis of pancreatic beta-cells in type 2 diabetes mellitus via regulation by miR-22 of the JAK/STAT signaling pathway. Diabetes Metab Syndr Obes. 2019;12:1645-1657. doi:10.2147/DMSO.S211700

97.Papazafiropoulou A, Perrea D, Moyssakis I, Kokkinos A, Katsilambros N, Tentolouris N. Plasma levels of MMP-2, MMP-9 and TIMP-1 are not associated with arterial stiffness in subjects with type 2 diabetes mellitus. J Diabetes Complications. 2010;24(1):20-27. doi:10.1016/j.jdiacomp.2008.10.004

98. Arndt T, Wedekind D, Jörns A, Tsiavaliaris G, Cuppen E, Hedrich HJ, Lenzen S. A novel Dock8 gene mutation confers diabetogenic susceptibility in the LEW.1AR1/Ztm-iddm rat, an animal model of human type 1 diabetes. Diabetologia. 2015;58(12):2800-2809. doi:10.1007/s00125-015-3757-7

99. Tabassum R, Mahajan A, Chauhan G, Dwivedi OP, Dubey H, Sharma V, Kundu B, Ghosh S, Tandon N, Bharadwaj D. No association of TNFRSF1B variants with type 2 diabetes in Indians of Indo-European origin. BMC Med Genet. 2011;12:110. doi:10.1186/1471-2350-12-110

100. Glawe JD, Patrick DR, Huang M, Sharp CD, Barlow SC, Kevil CG. Genetic deficiency of Itgb2 or ItgaL prevents autoimmune diabetes through 
distinctly different mechanisms in NOD/LtJ mice. Diabetes. 2009;58(6):1292-1301. doi:10.2337/db08-0804

101. Roberts DD, Isenberg JS. CD47 and thrombospondin-1 regulation of mitochondria, metabolism, and diabetes. Am J Physiol Cell Physiol. 2021;321(2):C201-C213. doi:10.1152/ajpcell.00175.2021

102. Zhang Y, Liu Y, Liu Y, Zhang Y, Su Z. Genetic Variants of Retinoic Acid Receptor-Related Orphan Receptor Alpha Determine Susceptibility to Type 2 Diabetes Mellitus in Han Chinese. Genes (Basel). 2016;7(8):54. doi:10.3390/genes7080054

103. Chibalin AV, Leng Y, Vieira E, Krook A, Björnholm M, Long YC, Kotova O, Zhong Z, Sakane F, Steiler T, et al. Downregulation of diacylglycerol kinase delta contributes to hyperglycemia-induced insulin resistance. Cell. 2008;132(3):375-386. doi:10.1016/j.cell.2007.12.035

104. Ding Y, Kantarci A, Badwey JA, Hasturk H, Malabanan A, Van Dyke TE. Phosphorylation of pleckstrin increases proinflammatory cytokine secretion by mononuclear phagocytes in diabetes mellitus. J Immunol. 2007;179(1):647-654. doi:10.4049/jimmunol.179.1.647

105. Lewis JP, Palmer ND, Ellington JB, Divers J, Ng MC, Lu L, Langefeld CD, Freedman BI, Bowden DW. Analysis of candidate genes on chromosome 20q12-13.1 reveals evidence for BMI mediated association of PREX1 with type 2 diabetes in European Americans. Genomics. 2010;96(4):211-219. doi:10.1016/j.ygeno.2010.07.006

106. Jin J, Cao L, Zhao Z, Shen S, Kiess W, Zhi D, Ye R, Cheng R, Chen L, Yang Y, et al. Novel BSCL2 gene mutation E189X in Chinese congenital generalized lipodystrophy child with early onset diabetes mellitus. Eur J Endocrinol. 2007;157(6):783-787. doi:10.1530/EJE-07-0393

107. Seref-Ferlengez Z, Maung S, Schaffler MB, Spray DC, Suadicani SO, Thi MM. P2X7R-Panx1 Complex Impairs Bone Mechanosignaling under High Glucose Levels Associated with Type-1 Diabetes. PLoS One. 2016;11(5):e0155107. doi:10.1371/journal.pone.0155107

108. Wang Z, Zheng Y, Hou C, Yang L, Li X, Lin J, Huang G, Lu Q, Wang CY, Zhou Z. DNA methylation impairs TLR9 induced Foxp3 expression by attenuating IRF-7 binding activity in fulminant type 1 diabetes. J Autoimmun. 2013;41:50-59. doi:10.1016/j.jaut.2013.01.009 
109. Wang Y, Wang S, Zhang W, Liu J, Yang Z, Liu C. Notch1 participates in the activation of autophagy in the hippocampus of type I diabetic mice. Neurochem Int. 2021;150:105156. doi:10.1016/j.neuint.2021.105156

110. Jardín I, López JJ, Zbidi H, Bartegi A, Salido GM, Rosado JA. Attenuated store-operated divalent cation entry and association between STIM1, Orai1, hTRPC1 and hTRPC6 in platelets from type 2 diabetic patients. Blood Cells Mol Dis. 2011;46(3):252-260. doi:10.1016/j.bcmd.2010.12.008

111. Qian Y, Lei G, Wen L. Brain-specific deletion of TRIM13 promotes metabolic stress-triggered insulin resistance, glucose intolerance, and neuroinflammation. Biochem Biophys Res Commun. 2020;527(1):138-145. doi:10.1016/j.bbrc.2020.03.076

112. Kardelen AD, Kara M, Güller D, Ozturan EK, Abalı ZY, Ceylaner S, Kiykım A, Cantez S, Torun SH, Poyrazoglu S, et al. LRBA deficiency: a rare cause of type 1 diabetes, colitis, and severe immunodeficiency. Hormones (Athens). 2021;20(2):389-394. doi:10.1007/s42000-020-00257-z

113. Zhang $\mathrm{H}$, Luan S, Xiao X, Lin L, Zhao X, Liu X. Silenced microRNA-222 suppresses inflammatory response in gestational diabetes mellitus mice by promoting CXCR4. Life Sci. 2021;266:118850. doi:10.1016/j.lfs.2020.118850

114. Pellegrino M, Traversi G, Arena A, Cappa M, Rosado MM, Andreani M, Delfino DV, Moretti F, Fierabracci A. Effect of p53 activation through targeting MDM2/MDM4 heterodimer on T regulatory and effector cells in the peripheral blood of Type 1 diabetes patients. PLoS One. 2020;15(1):e0228296. doi:10.1371/journal.pone.0228296

115. Santiago JL, Martínez A, Núñez C, de la Calle H, Fernández-Arquero M, de la Concha EG, Urcelay E. Association of MYO9B haplotype with type 1 diabetes. Hum Immunol. 2008;69(2):112-115. doi:10.1016/j.humimm.2008.01.003

116. Mátyás C, Németh BT, Oláh A, Török M, Ruppert M, Kellermayer D, Barta BA, Szabó G, Kökény G, Horváth EM, et al. Prevention of the development of heart failure with preserved ejection fraction by the phosphodiesterase-5A inhibitor vardenafil in rats with type 2 diabetes. Eur J Heart Fail. 2017;19(3):326-336. doi:10.1002/ejhf.711 
117. Gao W, Guo N, Zhao S, Chen Z, Zhang W, Yan F, Liao H, Chi K. FBXW7 promotes pathological cardiac hypertrophy by targeting EZH2SIX1 signaling. Exp Cell Res. 2020;393(1):112059. doi:10.1016/j.yexcr.2020.112059

118. Müller II, Müller KA, Karathanos A, Schönleber H, Rath D, Vogel S, Chatterjee M, Schmid M, Haas M, Seizer P, et al. Impact of counterbalance between macrophage migration inhibitory factor and its inhibitor Gremlin-1 in patients with coronary artery disease. Atherosclerosis. 2014;237(2):426432. doi:10.1016/j.atherosclerosis.2014.09.010

119. Kanashiro-Takeuchi RM, Takeuchi LM, Rick FG, Dulce R, Treuer AV, Florea V, Rodrigues CO, Paulino EC, Hatzistergos KE, Selem SM, et al. Activation of growth hormone releasing hormone (GHRH) receptor stimulates cardiac reverse remodeling after myocardial infarction (MI). Proc Natl Acad Sci U S A. 2012;109(2):559-563. doi:10.1073/pnas.1119203109

120. Mouat MA, Coleman JLJ, Wu J, Dos Remedios CG, Feneley MP, Graham RM, Smith NJ. Involvement of GPR37L1 in murine blood pressure regulation and human cardiac disease pathophysiology. Am J Physiol Heart Circ Physiol. 2021;321(4):H807-H817. doi:10.1152/ajpheart.00198.2021

121. Aliwarga T, Evangelista EA, Sotoodehnia N, Lemaitre RN, Totah RA.

Regulation of CYP2J2 and EET Levels in Cardiac Disease and Diabetes. Int J Mol Sci. 2018;19(7):1916. doi:10.3390/ijms19071916

122. Jiang Q, Dong X, Hu D, Chen L, Luo Y. Aquaporin 4 Inhibition Alleviates Myocardial Ischemia-Reperfusion Injury by Restraining Cardiomyocyte Pyroptosis. Bioengineered. 2021;10.1080/21655979.2021.1992332.

doi:10.1080/21655979.2021.1992332

123. Theodoraki EV, Nikopensius T, Suhorutsenko J, Papamikos V, Kolovou GD, Peppes V, Panagiotakos D, Limberi S, Zakopoulos N, Metspalu A, et al. ROS1 Asp2213Asn polymorphism is not associated with coronary artery disease in a Greek case-control study. Clin Chem Lab Med. 2009;47(12):1471-1473. doi:10.1515/CCLM.2009.155

124. Asada M, Oishi E, Sakata S, Hata J, Yoshida D, Honda T, Furuta Y, Shibata M, Suzuki K, Watanabe H, et al.Serum Lipopolysaccharide-Binding Protein Levels and the Incidence of Cardiovascular Disease in a General 
Japanese Population: The Hisayama Study. J Am Heart Assoc. 2019;8(21):e013628. doi:10.1161/JAHA.119.013628

125. Terranegra A, Arcidiacono T, Macrina L, Brasacchio C, Pivari F, Mingione A, Tomei S, Mezzavilla M, Silcock L, Cozzolino M, et al. Glucagon-like peptide-1 receptor and sarcoglycan delta genetic variants can affect cardiovascular risk in chronic kidney disease patients under hemodialysis. Clin Kidney J. 2020;13(4):666-673. doi:10.1093/ckj/sfz182

126. Liu D, Qiao C, Luo H. MicroRNA-1278 ameliorates the inflammation of cardiomyocytes during myocardial ischemia by targeting both IL-22 and CXCL14. Life Sci. 2021;269:118817. doi:10.1016/j.lfs.2020.118817

127. Yamaguchi M. Regulatory role of regucalcin in heart calcium signaling: Insight into cardiac failure (Review). Biomed Rep. 2014;2(3):303308. doi:10.3892/br.2014.245

128. Ameri A, Kurachi S, Sueishi K, Kuwahara M, Kurachi K. Myocardial fibrosis in mice with overexpression of human blood coagulation factor IX. Blood. 2003;101(5):1871-1873. doi:10.1182/blood-2002-05-1581

129. Perrin MJ, Adler A, Green S, Al-Zoughool F, Doroshenko P, Orr N, Uppal S, Healey JS, Birnie D, Sanatani S, et al. Evaluation of genes encoding for the transient outward current (Ito) identifies the KCND2 gene as a cause of J-wave syndrome associated with sudden cardiac death. Circ Cardiovasc Genet. 2014;7(6):782-789. doi:10.1161/CIRCGENETICS.114.000623

130. Huscher D, Ebert N, Soerensen-Zender I, Mielke N, Schaeffner E, Schmitt R. Development of a prediction model for mortality and cardiovascular outcomes in older adults taking into account AZGP1. Sci Rep. 2021;11(1):11792. doi:10.1038/s41598-021-91169-4

131. Msheik A, Kaspar C, Mailhac A, Hoballah JJ, Tamim H, Dakik HA. Performance of the AUB-HAS2 Cardiovascular Risk Index in vascular surgery patients. Vasc Med. 2021;26(5):535-541. doi:10.1177/1358863X21996806

132. McCarthy NS, Vangjeli C, Surendran P, Treumann A, Rooney C, Ho E, Sever P, Thom S, Hughes AD, Munroe PB, et al. Genetic variants in PPARGC1B and CNTN4 are associated with thromboxane A2 formation and with cardiovascular event free survival in the Anglo-Scandinavian 
Cardiac Outcomes Trial (ASCOT). Atherosclerosis. 2018;269:42-49. doi:10.1016/j.atherosclerosis.2017.12.013

133. Skaria T, Bachli E, Schoedon G. Transcriptional Regulation of Drug Metabolizing CYP Enzymes by Proinflammatory Wnt5A Signaling in Human Coronary Artery Endothelial Cells. Front Pharmacol. 2021;12:619588. doi:10.3389/fphar.2021.619588

134. Horne BD, Rasmusson KD, Alharethi R, Budge D, Brunisholz KD, Metz T, Carlquist JF, Connolly JJ, Porter TF, Lappé DL, et al. Genome-wide significance and replication of the chromosome 12p11.22 locus near the PTHLH gene for peripartum cardiomyopathy. Circ Cardiovasc Genet. 2011;4(4):359-366. doi:10.1161/CIRCGENETICS.110.959205

135. Tillmanns J, Hoffmann D, Habbaba Y, Schmitto JD, Sedding D,

Fraccarollo D, Galuppo P, Bauersachs J. Fibroblast activation protein alpha expression identifies activated fibroblasts after myocardial infarction. J Mol Cell Cardiol. 2015;87:194-203. doi:10.1016/j.yjmcc.2015.08.016

136. Li Y, Wang DW, Chen Y, Chen C, Guo J, Zhang S, Sun Z, Ding H, Yao Y, Zhou L, et al. Genome-Wide Association and Functional Studies Identify SCML4 and THSD7A as Novel Susceptibility Genes for Coronary Artery Disease. Arterioscler Thromb Vasc Biol. 2018;38(4):964-975. doi:10.1161/ATVBAHA.117.310594

137. Durbin MD, O'Kane J, Lorentz S, Firulli AB, Ware SM. SHROOM3 is downstream of the planar cell polarity pathway and loss-of-function results in congenital heart defects. Dev Biol. 2020;464(2):124-136. doi:10.1016/j.ydbio.2020.05.013

138. Rommel C, Rösner S, Lother A, Barg M, Schwaderer M, Gilsbach R, Bömicke T, Schnick T, Mayer S, Doll S, et al. The Transcription Factor ETV1 Induces Atrial Remodeling and Arrhythmia. Circ Res. 2018;123(5):550-563. doi:10.1161/CIRCRESAHA.118.313036

139. Qian P, Cao X, Xu X, Duan M, Zhang Q, Huang G. Contribution of CYP24A1 variants in coronary heart disease among the Chinese population. Lipids Health Dis. 2020;19(1):181. doi:10.1186/s12944-020-01356-X 140. Liu JW, Liu HT, Chen L. The Therapeutic Role of Slit2 in Antifibrosis, Anti-inflammation and Anti-oxidative Stress in Rats with Coronary Heart Disease. Cardiovasc Toxicol. 2021;21(12):973-983. doi:10.1007/s12012-021-09688-5 
141. Li RG, Xu YJ, Ye WG, Li YJ, Chen H, Qiu XB, Yang YQ, Bai D. Connexin45 (GJC1) loss-of-function mutation contributes to familial atrial fibrillation and conduction disease. Heart Rhythm. 2021;18(5):684-693. doi:10.1016/j.hrthm.2020.12.033

142. Nikitin AG, Chistiakov DA, Minushkina LO, Zateyshchikov DA, Nosikov VV. Association of the CYBA, PPARGC1A, PPARG3, and PPARD gene variants with coronary artery disease and metabolic risk factors of coronary atherosclerosis in a Russian population. Heart Vessels. 2010;25(3):229-236. doi:10.1007/s00380-009-1159-9

143. Pan RY, Liu P, Zhou HT, Sun WX, Song J, Shu J, Cui GJ, Yang ZJ, Jia EZ. Circular RNAs promote TRPM3 expression by inhibiting hsa-miR$130 \mathrm{a}-3 \mathrm{p}$ in coronary artery disease patients. Oncotarget. 2017;8(36):6028060290. doi:10.18632/oncotarget.19941

144. AsghariHanjani N, Vafa M. The role of IGF-1 in obesity, cardiovascular disease, and cancer. Med J Islam Repub Iran. 2019;33:56. doi:10.34171/mjiri.33.56

145. Zhang H, Ding P, Zheng L. TRPV6 Is Associated with Prognosis of ST-Elevation Acute Myocardial Infarction. Ann Clin Lab Sci. 2020;50(6):775-780.

146. Shao L, Zhang P, Zhang Y, Lu Q, Ma A. TLR3 and TLR4 as potential clinically biomarkers of cardiovascular risk in coronary artery disease (CAD) patients. Heart Vessels. 2014;29(5):690-698. doi:10.1007/s00380013-0421-3

147. Aluganti Narasimhulu C, Singla DK. The Role of Bone Morphogenetic Protein 7 (BMP-7) in Inflammation in Heart Diseases. Cells. 2020;9(2):280. doi:10.3390/cells9020280

148. Zou Y, Zhang Q, Zhang J, Chen X, Zhou W, Yang Z, Yang Q, Yu H, $\mathrm{Li} \mathrm{L}, \mathrm{He} \mathrm{Y}$, et al. A common indel polymorphism of the Desmoglein-2 (DSG2) is associated with sudden cardiac death in Chinese populations. Forensic Sci Int. 2019;301:382-387. doi:10.1016/j.forsciint.2019.06.008

149. Azharuddin M, Adil M, Ghosh P, Kapur P, Sharma M. Periostin as a novel biomarker of cardiovascular disease: A systematic evidence landscape of preclinical and clinical studies. J Evid Based Med. 2019;12(4):325-336. doi:10.1111/jebm.12368 
150. Zhang R, Ji Z, Cai J, Li Y, Ma G. Clinical Significance of Serum Kallistatin and ENOX1 Levels in Patients with Coronary Heart Disease. Med Princ Pract. 2021;30(4):339-346. doi:10.1159/000510427

151. Bardien-Kruger S, Wulff H, Arieff Z, Brink P, Chandy KG, Corfield V. Characterisation of the human voltage-gated potassium channel gene, KCNA7, a candidate gene for inherited cardiac disorders, and its exclusion as cause of progressive familial heart block I (PFHBI). Eur J Hum Genet. 2002;10(1):36-43. doi:10.1038/sj.ejhg.5200739

152. Khatami M, Heidari MM, Soheilyfar S. Common rs5918 (PlA1/A2) polymorphism in the ITGB3 gene and risk of coronary artery disease. Arch Med Sci Atheroscler Dis. 2016;1(1):e9-e15. doi:10.5114/amsad.2016.59587

153. Vanhoutte D, Schips TG, Vo A, Grimes KM, Baldwin TA, Brody MJ, Accornero F, Sargent MA, Molkentin JD. Thbs1 induces lethal cardiac atrophy through PERK-ATF4 regulated autophagy. Nat Commun. 2021;12(1):3928. doi:10.1038/s41467-021-24215-4

154. Seo K, Rainer PP, Shalkey Hahn V, Lee DI, Jo SH, Andersen A, Liu T, Xu X, Willette RN, Lepore JJ, et al. Combined TRPC3 and TRPC6 blockade by selective small-molecule or genetic deletion inhibits pathological cardiac hypertrophy. Proc Natl Acad Sci U S A. 2014;111(4):1551-1556. doi:10.1073/pnas.1308963111

155. Reiner AP, Carlson CS, Thyagarajan B, Rieder MJ, Polak JF, Siscovick DS, Nickerson DA, Jacobs DR Jr, Gross MD. Soluble P-selectin, SELP polymorphisms, and atherosclerotic risk in European-American and African-African young adults: the Coronary Artery Risk Development in Young Adults (CARDIA) Study. Arterioscler Thromb Vasc Biol. 2008;28(8):1549-1555. doi:10.1161/ATVBAHA.108.169532

156. Hawley MH, Almontashiri N, Biesecker LG, Berger N, Chung WK, Garcia J, Grebe TA, Kelly MA, Lebo MS, Macaya D, et al. An assessment of the role of vinculin loss of function variants in inherited cardiomyopathy. Hum Mutat. 2020;41(9):1577-1587. doi:10.1002/humu.24061

157. Ueda Y, Li RHL, Nguyen N, Ontiveros ES, Kovacs SL, Oldach MS, Vernau KM, Court MH, Stern JA. A genetic polymorphism in P2RY1 impacts response to clopidogrel in cats with hypertrophic cardiomyopathy. Sci Rep. 2021;11(1):12522. doi:10.1038/s41598-021-91372-3 
158. Geng H, Guan J. MiR-18a-5p inhibits endothelial-mesenchymal transition and cardiac fibrosis through the Notch2 pathway. Biochem Biophys Res Commun. 2017;491(2):329-336. doi:10.1016/j.bbrc.2017.07.101

159. Gao BF, Shen ZC, Bian WS, Wu SX, Kang ZX, Gao Y. Correlation of hypertension and F2RL3 gene methylation with Prognosis of coronary heart disease. J Biol Regul Homeost Agents. 2018;32(6):1539-1544.

160. Nicholson AM, Finch NA, Almeida M, Perkerson RB, van Blitterswijk M, Wojtas A, Cenik B, Rotondo S, Inskeep V, Almasy L, et al. Prosaposin is a regulator of progranulin levels and oligomerization. Nat Commun. 2016;7:11992. doi:10.1038/ncomms1199

161. Konaniah ES, Kuhel DG, Basford JE, Weintraub NL, Hui DY. Deficiency of LRP1 in Mature Adipocytes Promotes Diet-Induced Inflammation and Atherosclerosis-Brief Report. Arterioscler Thromb Vasc Biol. 2017;37(6):1046-1049. doi:10.1161/ATVBAHA.117.309414

162. Koller L, Richter B, Winter MP, Sulzgruber P, Potolidis C, Liebhart F, Mörtl D, Berger R, Goliasch G, Lang I, et al. Clusterin/apolipoprotein J is independently associated with survival in patients with chronic heart failure. J Clin Lipidol. 2017;11(1):178-184. doi:10.1016/j.jacl.2016.11.009

163. Mo XG, Liu W, Yang Y, Imani S, Lu S, Dan G, Nie X, Yan J, Zhan R, Li X, et al. NCF2, MYO1F, S1PR4, and FCN1 as potential noninvasive diagnostic biomarkers in patients with obstructive coronary artery: A weighted gene co-expression network analysis. J Cell Biochem. 2019;120(10):18219-18235. doi:10.1002/jcb.29128

164. Rodríguez I, Coto E, Reguero JR, González P, Andrés V, Lozano I, Martín M, Alvarez V, Morís C. Role of the CDKN1A/p21, CDKN1C/p57, and $\mathrm{CDKN} 2 \mathrm{~A} / \mathrm{p} 16$ genes in the risk of atherosclerosis and myocardial infarction. Cell Cycle. 2007;6(5):620-625. doi:10.4161/cc.6.5.3927

165. Gong L, Zhu L, Yang T. Fendrr involves in the pathogenesis of cardiac fibrosis via regulating miR-106b/SMAD3 axis. Biochem Biophys Res Commun. 2020;524(1):169-177. doi:10.1016/j.bbrc.2020.01.062

166. Chelko SP, Keceli G, Carpi A, Doti N, Agrimi J, Asimaki A, Beti CB, Miyamoto M, Amat-Codina N, Bedja D, et al. Exercise triggers CAPN1mediated AIF truncation, inducing myocyte cell death in arrhythmogenic 
cardiomyopathy. Sci Transl Med. 2021;13(581):eabf0891. doi:10.1126/scitranslmed.abf0891

167. Bayoumi AS, Park KM, Wang Y, Teoh JP, Aonuma T, Tang Y, Su H, Weintraub NL, Kim IM. A carvedilol-responsive microRNA, miR-125b-5p protects the heart from acute myocardial infarction by repressing proapoptotic bak1 and klf13 in cardiomyocytes. J Mol Cell Cardiol. 2018;114:72-82. doi:10.1016/j.yjmcc.2017.11.003

168. Oksala N, Pärssinen J, Seppälä I, Klopp N, Illig T, Laaksonen R, Levula M, Raitoharju E, Kholova I, Sioris T, et al. Kindlin 3 (FERMT3) is associated with unstable atherosclerotic plaques, anti-inflammatory type II macrophages and upregulation of beta- 2 integrins in all major arterial beds. Atherosclerosis.

2015;242(1):145-154.

doi:10.1016/j.atherosclerosis.2015.06.058

169. Zidi I, Kharrat N, Abdelhedi R, Hassine AB, Laaribi AB, Yahia HB, Abdelmoula NB, Abid L, Rebai A, Rizzo R. Nonclassical human leukocyte antigen (HLA-G, HLA-E, and HLA-F) in coronary artery disease. Hum Immunol. 2016;77(4):325-329. doi:10.1016/j.humimm.2016.01.008

170. Safranow K, Rzeuski R, Binczak-Kuleta A, Czyzycka E, Skowronek J, Jakubowska K, Wojtarowicz A, Loniewska B, et al. ADA*2 allele of the adenosine deaminase gene may protect against coronary artery disease. Cardiology. 2007;108(4):275-281. doi:10.1159/000099096

171. Thude H, Gerlach K, Richartz B, Krack A, Brenke B, Pethig K, Figulla HR, Barz D. No association between transmembrane proteintyrosine phosphatase receptor type $\mathrm{C}$ (CD45) exon A point mutation $(77 \mathrm{C}>\mathrm{G})$ and idiopathic dilated cardiomyopathy. Hum Immunol. 2005;66(9):1008-1012. doi:10.1016/j.humimm.2005.07.004

172. Ansari N, Najafi S, Shahrabi S, Saki N. PEAR1 polymorphisms as a prognostic factor in hemostasis and cardiovascular diseases. J Thromb Thrombolysis. 2021;51(1):89-95. doi:10.1007/s11239-020-02149-w

173. Lu Y, Boer JM, Barsova RM, Favorova O, Goel A, Müller M,

Feskens EJ. TGFB1 genetic polymorphisms and coronary heart disease risk: a meta-analysis. BMC Med Genet. 2012;13:39. doi:10.1186/1471-2350-1339

174. García-Bermúdez M, López-Mejías R, Genre F, Castañeda S, Corrales A, Llorca J, González-Juanatey C, Ubilla B, Miranda-Filloy JA, et al. Lack 
of association between JAK3 gene polymorphisms and cardiovascular disease in Spanish patients with rheumatoid arthritis. Biomed Res Int. 2015;2015:318364. doi:10.1155/2015/318364

175. Kim JY, Choi BG, Jelinek J, Kim DH, Lee SH, Cho K, Rha SH, Lee YH, Jin HS, Choi DK, et al. Promoter methylation changes in ALOX12 and AIRE1: novel epigenetic markers for atherosclerosis. Clin Epigenetics. 2020;12(1):66. doi:10.1186/s13148-020-00846-0

176. Liu L, Zhao Q, Kong M, Mao L, Yang Y, Xu Y. Myocardin-related transcription factor A (MRTF-A) regulates integrin beta 2 transcription to promote macrophage infiltration and cardiac hypertrophy in mice. Cardiovasc Res. 2021;cvab110. doi:10.1093/cvr/cvab110

177. Tregouet DA, Barbaux S, Poirier O, Blankenberg S, Bickel C, Escolano S, Rupprecht HJ, Meyer J, Cambien F, Tiret L. SELPLG gene polymorphisms in relation to plasma SELPLG levels and coronary artery disease. Ann Hum Genet. 2003;67(Pt 6):504-511. doi:10.1046/j.15298817.2003.00053.x

178. Liu S, Wang X, Yu S, Yan M, Peng Y, Zhang G, Xu Z. A MetaAnalysis on the Association Between TNFSF4 Polymorphisms (rs3861950 $\mathrm{T}>\mathrm{C}$ and rs1234313 A > G) and Susceptibility to Coronary Artery Disease.

Front Physiol. 2020;11:539288. doi:10.3389/fphys.2020.539288

179. Mitchell GF, Verwoert GC, Tarasov KV, Isaacs A, Smith AV, Yasmin, Rietzschel ER, Tanaka T, Liu Y, Parsa A, et al. Common genetic variation in the 3'-BCL11B gene desert is associated with carotid-femoral pulse wave velocity and excess cardiovascular disease risk: the AortaGen Consortium. Circ Cardiovasc Genet. 2012;5(1):81-90. doi:10.1161/CIRCGENETICS.111.959817

180. Coto E, Pascual I, Avanzas P, Cuesta-Lavona E, Lorca R, Martín M, Vázquez-Coto D, Díaz-Corte $\mathrm{C}$, Morís $\mathrm{C}$, Rodríguez-Reguero $\mathrm{J}$, et al. IL17RA in early-onset coronary artery disease: Total leukocyte transcript analysis and promoter polymorphism (rs4819554) association. Cytokine. 2020;136:155285. doi:10.1016/j.cyto.2020.155285

181. Ding Y, Xu Z, Pan Y, Meng X, Xiang X, Li H, Liu L, Wang Y. Association Between CST3 Gene Polymorphisms and Large-Artery Atherosclerotic Stroke. Front Neurol. 2021;12:738148. doi:10.3389/fneur.2021.738148 
182. Prasongsukarn K, Dechkhajorn W, Benjathummarak S, Maneerat Y. TRPM2, PDLIM5, BCL3, CD14, GBA Genes as Feasible Markers for Premature Coronary Heart Disease Risk. Front Genet. 2021;12:598296. doi:10.3389/fgene.2021.598296

183. Benjafield AV, Wang XL, Morris BJ. Tumor necrosis factor receptor 2 gene (TNFRSF1B) in genetic basis of coronary artery disease. J Mol Med (Berl). 2001;79(2-3):109-115. doi:10.1007/s001090000168

184. Crawford AA, Bankier S, Altmaier E, Barnes CLK, Clark DW, Ermel R, Friedrich N, van der Harst P, Joshi PK, Karhunen V, et al. Variation in the SERPINA6/SERPINA1 locus alters morning plasma cortisol, hepatic corticosteroid binding globulin expression, gene expression in peripheral tissues, and risk of cardiovascular disease. J Hum Genet. 2021;66(6):625636. doi:10.1038/s10038-020-00895-6

185. Cheng Q, Gu J, Adhikari BK, Sun L, Sun J. Is CD47 a potentially promising therapeutic target in cardiovascular diseases? - Role of CD47 in cardiovascular diseases. Life Sci. 2020;247:117426. doi:10.1016/j.lfs.2020.117426

186. Cheng WL, Kao YH, Chen YC, Lin YK, Chen SA, Chen YJ. Macrophage migration inhibitory factor increases atrial arrhythmogenesis through CD74 signaling. Transl Res. 2020;216:43-56. doi:10.1016/j.trsl.2019.10.002

187. Yamada Y, Sakuma J, Takeuchi I, Yasukochi Y, Kato K, Oguri M, Fujimaki T, Horibe H, Muramatsu M, Sawabe M, et al. Identification of STXBP2 as a novel susceptibility locus for myocardial infarction in Japanese individuals by an exome-wide association study. Oncotarget. 2017;8(20):33527-33535. doi:10.18632/oncotarget.16536

188. Lai CH, Pandey S, Day CH, Ho TJ, Chen RJ, Chang RL, Pai PY, Padma VV, Kuo WW, Huang CY. $\beta$-catenin/LEF1/IGF-IIR Signaling Axis Galvanizes the Angiotensin-II- induced Cardiac Hypertrophy. Int J Mol Sci. 2019;20(17):4288. doi:10.3390/ijms20174288

189. Carreras-Torres R, Athanasiadis G, Via M, Trenchs J, Gayà-Vidal M, Santamaria J, Esteban E, Moral P. Allele-allele interaction within the F13A1 gene: a risk factor for ischaemic heart disease in Spanish population. Thromb Res. 2010;126(3):e241-e245. doi:10.1016/j.thromres.2010.04.021 
190. Zhou H, Lei X, Yan Y, Lydic T, Li J, Weintraub NL, Su H, Chen W. Targeting ATGL to rescue BSCL2 lipodystrophy and its associated cardiomyopathy. JCI Insight. 2019;5(14):e129781. doi:10.1172/jci.insight.129781

191. Chabior A, Gąsecka A, Marchel M, Gozdowska R, Makowska A, Maciak K, Góra M, Filipiak KJ, Burzyńska B, Opolski G. Expression of versican mRNA transcript to predict cardiac remodelling after myocardial infarction. Kardiol Pol. 2021;79(7-8):833-840. doi:10.33963/KP.a2021.0042

192. Stewart RM, Rodriguez EC, King MC. Ablation of SUN2-containing LINC complexes drives cardiac hypertrophy without interstitial fibrosis. Mol Biol Cell. 2019;30(14):1664-1675. doi:10.1091/mbc.E18-07-0438

193. Wang B, Zhou Q, Bi Y, Zhou W, Zeng Q, Liu Z, Liu X, Zhan Z. Phosphatase PPM1L Prevents Excessive Inflammatory Responses and Cardiac Dysfunction after Myocardial Infarction by Inhibiting IKK $\beta$ Activation. J Immunol. 2019;203(5):1338-1347. doi:10.4049/jimmunol.1900148

194. Jiang DS, Liu Y, Zhou H, Zhang Y, Zhang XD, Zhang XF, Chen K, Gao L, Peng J, Gong H, et al. Interferon regulatory factor 7 functions as a novel negative regulator of pathological cardiac hypertrophy. Hypertension. 2014;63(4):713-722. doi:10.1161/HYPERTENSIONAHA.113.02653

195. Chen X, Ren L, Liu X, Sun X, Dong C, Jiang Y, Qin Y, Qu H, Jiao J, Wang S, et al. Ranolazine protects against diabetic cardiomyopathy by activating the NOTCH1/NRG1 pathway. Life Sci. 2020;261:118306. doi:10.1016/j.lfs.2020.118306

196. Gudmundsson H, Curran J, Kashef F, Snyder JS, Smith SA, VargasPinto P, Bonilla IM, Weiss RM, Anderson ME, Binkley P, et al. Differential regulation of EHD3 in human and mammalian heart failure. J Mol Cell Cardiol. 2012;52(5):1183-1190. doi:10.1016/j.yjmcc.2012.02.008

197. Perisic L, Hedin E, Razuvaev A, Lengquist M, Osterholm C, Folkersen L, Gillgren P, Paulsson-Berne G, Ponten F, Odeberg J, Hedin U. Profiling of atherosclerotic lesions by gene and tissue microarrays reveals PCSK6 as a novel protease in unstable carotid atherosclerosis. Arterioscler Thromb Vasc Biol. 2013;33(10):2432-2443. doi:10.1161/ATVBAHA.113.301743 
198. Correll RN, Goonasekera SA, van Berlo JH, Burr AR, Accornero F, Zhang H, Makarewich CA, York AJ, Sargent MA, Chen X, et al. STIM1 elevation in the heart results in aberrant $\mathrm{Ca}^{2} \square$ handling and cardiomyopathy. J Mol Cell Cardiol. 2015;87:38-47. doi:10.1016/j.yjmcc.2015.07.032

199. Weiberg D, Thackeray JT, Daum G, Sohns JM, Kropf S, Wester HJ, Ross TL, Bengel FM, Derlin T. Clinical Molecular Imaging of Chemokine Receptor CXCR4 Expression in Atherosclerotic Plaque Using 68GaPentixafor PET: Correlation with Cardiovascular Risk Factors and Calcified Plaque Burden. J Nucl Med. 2018;59(2):266-272. doi:10.2967/jnumed.117.196485

200. Angrisano T, Schiattarella GG, Keller S, Pironti G, Florio E, Magliulo F, Bottino R, Pero R, Lembo F, Avvedimento EV, et al. Epigenetic switch at atp2a2 and myh7 gene promoters in pressure overload-induced heart failure. PLoS One. 2014;9(9):e106024. doi:10.1371/journal.pone.0106024

201. Gomez-Hurtado N, Boczek NJ, Kryshtal DO, Johnson CN, Sun J, Nitu FR, Cornea RL, Chazin WJ, Calvert ML, Tester DJ, et al. Novel CPVT-Associated Calmodulin Mutation in CALM3 (CALM3-A103V) Activates Arrhythmogenic $\mathrm{Ca}$ Waves and Sparks. Circ Arrhythm Electrophysiol. 2016;9(8):10.1161/CIRCEP.116.004161 e004161. doi:10.1161/CIRCEP.116.004161

202. Wei Y, Zhu M, Corbalán-Campos J, Heyll K, Weber C, Schober A. Regulation of Csf1r and Bcl6 in macrophages mediates the stage-specific effects of microRNA-155 on atherosclerosis. Arterioscler Thromb Vasc Biol. 2015;35(4):796-803. doi:10.1161/ATVBAHA.114.304723

203. Bhaskaran S, Pharaoh G, Ranjit R, Murphy A, Matsuzaki S, Nair BC, Forbes B, Gispert S, Auburger G, Humphries KM, et al. Loss of mitochondrial protease ClpP protects mice from diet-induced obesity and insulin resistance. EMBO Rep. 2018;19(3):e45009. doi:10.15252/embr.201745009

204. Song HK, Kim J, Lee JS, Nho KJ, Jeong HC, Kim J, Ahn Y, Park WJ, Kim DH. Pik3ip1 modulates cardiac hypertrophy by inhibiting PI3K pathway. PLoS One. 2015;10(3):e0122251. doi:10.1371/journal.pone.0122251 
205. Krystof V, Chamrád I, Jorda R, Kohoutek J. Pharmacological targeting of CDK9 in cardiac hypertrophy. Med Res Rev. 2010;30(4):646666. doi:10.1002/med.20172

206. Ma S, Sun L, Wu W, Wu J, Sun Z, Ren J. USP22 Protects Against Myocardial Ischemia-Reperfusion Injury via the SIRT1-p53/SLC7A11Dependent Inhibition of Ferroptosis-Induced Cardiomyocyte Death. Front Physiol. 2020;11:551318. doi:10.3389/fphys.2020.551318

207. Zhang H, Wang J, Du A, Li Y. MiR-483-3p inhibition ameliorates myocardial ischemia/reperfusion injury by targeting the MDM4/p53 pathway. Mol Immunol. 2020;125:9-14. doi:10.1016/j.molimm.2020.06.014 208. Navarro-Núñez L, Roldán V, Lozano ML, Rivera J, Marin F, Vicente V, Martínez C. TUBB1 Q43P polymorphism does not protect against acute coronary syndrome and premature myocardial infarction. Thromb Haemost. 2008;100(6):1211-1213.

209. Maryam A, Khalid RR, Siddiqi AR, Ece A. E-pharmacophore based virtual screening for identification of dual specific PDE5A and PDE3A inhibitors as potential leads against cardiovascular diseases. J Biomol Struct Dyn. 2021;39(7):2302-2317. doi:10.1080/07391102.2020.1748718

210. Sciacca FL, Ciaccio C, Fontana F, Strano C, Gilardoni F, Pantaleoni C, D'Arrigo S. Severe Phenotype in a Patient With Homozygous 15q21.2 Microdeletion Involving BCL2L10, GNB5, and MYO5C Genes, Resembling Infantile Developmental Disorder With Cardiac Arrhythmias (IDDCA). Front Genet. 2020;11:399. doi:10.3389/fgene.2020.00399

211. Polonikov AV, Ponomarenko IV, Bykanova MA, Sirotina SS, Bocharova AV, Vagaytseva KV, Stepanov VA, Azarova IE, Churnosov MI, Solodilova MA. A comprehensive study revealed SNP-SNP interactions and a sex-dependent relationship between polymorphisms of the CYP2J2 gene and hypertension risk. Hypertens Res. 2019;42(2):257-272. doi:10.1038/s41440-018-0142-1

212. Chida A, Shintani M, Nakayama T, Furutani Y, Hayama E, Inai K, Saji T, Nonoyama S, Nakanishi T. Missense mutations of the BMPR1B (ALK6) gene in childhood idiopathic pulmonary arterial hypertension. Circ J. 2012;76(6):1501-1508. doi:10.1253/circj.cj-11-1281

213. Yamada Y, Kato K, Yoshida T, Yokoi K, Matsuo H, Watanabe S, Ichihara S, Metoki N, Yoshida $\mathrm{H}$, Satoh $\mathrm{K}$, et al. Association of 
polymorphisms of ABCA1 and ROS1 with hypertension in Japanese individuals. Int J Mol Med. 2008;21(1):83-89.

214. Zhang B, Li M, Wang L, Li C, Lou Y, Liu J, Liu Y, Wang Z, Wen S. The association between the polymorphisms in a sodium channel gene SCN7A and essential hypertension: a case-control study in the Northern Han Chinese. Ann Hum Genet. 2015;79(1):28-36. doi:10.1111/ahg. 12085

215. Kawarazaki W, Mizuno R, Nishimoto M, Ayuzawa N, Hirohama D, Ueda K, Kawakami-Mori F, Oba S, Marumo T, Fujita T. Salt causes agingassociated hypertension via vascular Wnt5a under Klotho deficiency. J Clin Invest. 2020;130(8):4152-4166. doi:10.1172/JCI134431

216. Ueda K, Nishimoto M, Hirohama D, Ayuzawa N, Kawarazaki W, Watanabe A, Shimosawa T, Loffing J, Zhang MZ, Marumo T, et al. Renal Dysfunction Induced by Kidney-Specific Gene Deletion of Hsd11b2 as a Primary Cause of Salt-Dependent Hypertension. Hypertension. 2017;70(1):111-118. doi:10.1161/HYPERTENSIONAHA.116.08966

217. Bao Q, Wang D, Zhang Y, Bao L, Jia H. The Impact of CYP24A1 Polymorphisms on Hypertension Susceptibility. Kidney Blood Press Res. 2020;45(1):28-37. doi:10.1159/000503925

218. Leng Q, Kahle KT, Rinehart J, MacGregor GG, Wilson FH, Canessa CM, Lifton RP, Hebert SC. WNK3, a kinase related to genes mutated in hereditary hypertension with hyperkalaemia, regulates the $\mathrm{K}+$ channel ROMK1 (Kir1.1). J Physiol. 2006;571(Pt 2):275-286. doi:10.1113/jphysiol.2005.102202

219. Taghvaei S, Saremi L, Babaniamansour S. Computational Analysis of Gly482Ser Single-Nucleotide Polymorphism in PPARGC1A Gene Associated with CAD, NAFLD, T2DM, Obesity, Hypertension, and Metabolic Diseases. PPAR Res. 2021;2021:5544233. doi:10.1155/2021/5544233

220. Norling AM, Gerstenecker AT, Buford TW, Khan B, Oparil S, Lazar RM. The role of exercise in the reversal of IGF-1 deficiencies in microvascular rarefaction and hypertension. Geroscience. 2020;42(1):141158. doi:10.1007/s11357-019-00139-2

221. Brown A, Meor Azlan NF, Wu Z, Zhang J. WNK-SPAK/OSR1-NCC kinase signaling pathway as a novel target for the treatment of salt-sensitive 
hypertension. Acta Pharmacol Sin. 2021;42(4):508-517. doi:10.1038/s41401-020-0474-7

222. Singh MV, Cicha MZ, Nunez S, Meyerholz DK, Chapleau MW, Abboud FM. Angiotensin II-induced hypertension and cardiac hypertrophy are differentially mediated by TLR3- and TLR4-dependent pathways. Am J Physiol Heart Circ Physiol. 2019;316(5):H1027-H1038. doi:10.1152/ajpheart.00697.2018

223. Liu D, Wu BX, Sun N, Yan Y, Yuan P, Qu JM, Jing ZC. Elevated Levels of Circulating Bone Morphogenetic Protein 7 Predict Mortality in Pulmonary Arterial Hypertension. Chest. 2016;150(2):367-373. doi:10.1016/j.chest.2016.03.007

224. Nie X, Shen C, Tan J, Wu Z, Wang W, Chen Y, Dai Y, Yang X, Ye S, Chen J, et al. Periostin: A Potential Therapeutic Target For Pulmonary Hypertension?. Circ Res. 2020;127(9):1138-1152. doi:10.1161/CIRCRESAHA.120.316943

225. Liu A, Liu Y, Li B, Yang M, Liu Y, Su J. Role of miR-223-3p in pulmonary arterial hypertension via targeting ITGB3 in the ECM pathway. Cell Prolif. 2019;52(2):e12550. doi:10.1111/cpr.12550

226. Sharma K, Chanana N, Mohammad G, Thinlas T, Gupta M, Syed MA, Das RS, Pasha Q, Mishra A. Hypertensive Patients Exhibit Enhanced Thrombospondin-1 Levels at High-Altitude. Life (Basel). 2021;11(9):893. doi:10.3390/life11090893

227. Liu C, Tang W, Zhao H, Yang S, Ren Z, Li J, Chen Y, Zhao X, Xu D,

Zhao Y, et al. The variants at FLNA and FLNB contribute to the susceptibility of hypertension and stroke with differentially expressed mRNA. Pharmacogenomics J. 2021;21(4):458-466. doi:10.1038/s41397021-00222-y

228. Jain PP, Lai N, Xiong M, Chen J, Babicheva A, Zhao T, Parmisano S, Zhao M, Paquin C, Matti M, et al. TRPC6, a Therapeutic Target for Pulmonary Hypertension. Am J Physiol Lung Cell Mol Physiol. 2021;10.1152/ajplung.00159.2021. doi:10.1152/ajplung.00159.2021

229. Novoyatleva T, Kojonazarov B, Owczarek A, Veeroju S, Rai N, Henneke I, Böhm M, Grimminger F, Ghofrani HA, Seeger W, Weissmann N, et al. Evidence for the Fucoidan/P-Selectin Axis as a Therapeutic Target 
in Hypoxia-induced Pulmonary Hypertension. Am J Respir Crit Care Med. 2019;199(11):1407-1420. doi:10.1164/rccm.201806-1170OC

230. Yu Q, Sun HP, Chen WQ, Chen XQ, Xu Y, He YH, Kong QP. Sexspecific association of rs4746172 of VCL gene with hypertension in two Han populations from Southern China. Sci Rep. 2015;5:15245. doi:10.1038/srep15245

231. Sahoo S, Li Y, de Jesus D, Sembrat J, Rojas MM, Goncharova E, Cifuentes-Pagano E, Straub AC, Pagano PJ. Notch2 suppression mimicking changes in human pulmonary hypertension modulates Notch1 and promotes endothelial cell proliferation. Am J Physiol Heart Circ Physiol. 2021;321(3):H542-H557. doi:10.1152/ajpheart.00125.2021

232. Gamboa R, Jaramillo-Estrella MJ, Martínez-Alvarado MDR, Soto ME, Torres-Paz YE, Gonzalo-Calvo D, Del Valle-Mondragón L, LópezMarure R, Llorente-Cortés VC, Huesca-Gómez C. Monocyte Low-Density Lipoprotein Receptor-Related Protein 1 (LRP1) Expression Correlates with cIMT in Mexican Hypertensive Patients. Expressão de Proteína-1 Relacionada a Receptor de Lipoproteína de Baixa Densidade (LRP1) em Monócito em Correlação com EIMC em Pacientes Mexicanos Hipertensos. Arq Bras Cardiol. 2021;116(1):56-65. doi:10.36660/abc.20190535

233. Zeng S, Han M, Jiang M, Liu F, Hu Y, Long Y, Zhu C, Zeng F, Gan $\mathrm{Q}$, Ye W, et al. Serum complement proteomics reveal biomarkers for hypertension disorder of pregnancy and the potential role of Clusterin. Reprod Biol Endocrinol. 2021;19(1):56. doi:10.1186/s12958-021-00742-Z

234. Zabini D, Granton E, Hu Y, Miranda MZ, Weichelt U, Breuils Bonnet S, Bonnet S, Morrell NW, Connelly KA, et al. Loss of SMAD3 Promotes Vascular Remodeling in Pulmonary Arterial Hypertension via MRTF Disinhibition. Am J Respir Crit Care Med. 2018;197(2):244-260. doi:10.1164/rcem.201702-0386OC

235. Olivi L, Vandenbriele C, Gu YM, Salvi E, Carpini SD, Liu YP, Jacobs L, Jin Y, Thijs L, Citterio L, et al. PEAR1 is not a human hypertension-susceptibility gene. Blood Press. 2015;24(1):61-64. doi:10.3109/08037051.2014.986928

236. Liu L, Wang C, Mi Y, Liu D, Li L, Fan J, Nan L, Jia N, Du Y. Association of MYH9 Polymorphisms with Hypertension in Patients with 
Chronic Kidney Disease in China. Kidney Blood Press Res. 2016;41(6):956965. doi:10.1159/000452597

237. Nabrdalik K, Gumprecht J, Adamczyk P, Górczyńska-Kosiorz S, Zywiec J, Grzeszczak W. Association of rs1800471 polymorphism of TGFB1 gene with chronic kidney disease occurrence and progression and hypertension appearance. Arch Med Sci. 2013;9(2):230-237. doi:10.5114/aoms.2013.34418

238. Katayose D, Ohe M, Yamauchi K, Ogata M, Shirato K, Fujita H, Shibahara S, Takishima T. Increased expression of PDGF A- and B-chain genes in rat lungs with hypoxic pulmonary hypertension. Am J Physiol. 1993;264(2 Pt 1):L100-L106. doi:10.1152/ajplung.1993.264.2.L100

239. Topchieva LV, Korneva VA, Kurbatova IV. The relationship of the carriership of allelic variations in rs2228145 (A > C) of the IL6R gene with the levels of VCAM1 and ICAM1 gene transcripts in patients with essential hypertension. Vavilovskii Zhurnal Genet Selektsii. 2020;24(1):96-101. doi:10.18699/VJ20.600

240. Onal IK, Altun B, Onal ED, Kirkpantur A, Gul Oz S, Turgan C. Serum levels of MMP-9 and TIMP-1 in primary hypertension and effect of antihypertensive treatment. Eur J Intern Med. 2009;20(4):369-372. doi:10.1016/j.ejim.2008.10.003

241. Speirs HJ, Wang WY, Benjafield AV, Morris BJ. No association with hypertension of CLCNKB and TNFRSF1B polymorphisms at a hypertension locus on chromosome 1p36. J Hypertens. 2005;23(8):14911496. doi:10.1097/01.hjh.0000174300.73992.cc

242. Novelli EM, Little-Ihrig L, Knupp HE, Rogers NM, Yao M, Baust JJ, Meijles D, St Croix CM, Ross MA, Pagano PJ, et al. Vascular TSP1-CD47 signaling promotes sickle cell-associated arterial vasculopathy and pulmonary hypertension in mice. Am J Physiol Lung Cell Mol Physiol. 2019;316(6):L1150-L1164. doi:10.1152/ajplung.00302.2018

243. Le Hiress M, Tu L, Ricard N, Phan C, Thuillet R, Fadel E, Dorfmüller P, Montani D, de Man F, Humbert M, et al. Proinflammatory Signature of the Dysfunctional Endothelium in Pulmonary Hypertension. Role of the Macrophage Migration Inhibitory Factor/CD74 Complex. Am J Respir Crit Care Med. 2015;192(8):983-997. doi:10.1164/rccm.201402-0322OC 
244. Chang YT, Chan CK, Eriksson I, Johnson PY, Cao X, Westöö C, Norvik C, Andersson-Sjöland A, Westergren-Thorsson G, Johansson S, et al. Versican accumulates in vascular lesions in pulmonary arterial hypertension. Pulm Circ. 2016;6(3):347-359. doi:10.1086/686994

245. Deng Y, Guo SL, Li JQ, Xie SS, Zhou YC, Wei B, Wang Q, Wang F. Interferon regulatory factor 7 inhibits rat vascular smooth muscle cell proliferation and inflammation in monocrotaline-induced pulmonary hypertension. Life Sci. 2021;264:118709. doi:10.1016/j.lfs.2020.118709

246. Wang S, Zhu G, Jiang D, Rhen J, Li X, Liu H, Lyu Y, Tsai P, Rose Y, Nguyen T, et al. Reduced Notch1 Cleavage Promotes the Development of Pulmonary Hypertension. Hypertension. 2021;HYPERTENSIONAHA12016065.

doi:10.1161/HYPERTENSIONAHA.120.16065

247. Dhande IS, Zhu Y, Kneedler SC, Joshi AS, Hicks MJ, Wenderfer SE, Braun MC, Doris PA. Stim1 Polymorphism Disrupts Immune Signaling and Creates Renal Injury in Hypertension. $J$ Am Heart Assoc. 2020;9(5):e014142. doi:10.1161/JAHA.119.014142

248. Yu BB, Zhi H, Zhang XY, Liang JW, He J, Su C, Xia WH, Zhang GX, Tao J. Mitochondrial dysfunction-mediated decline in angiogenic capacity of endothelial progenitor cells is associated with capillary rarefaction in patients with hypertension via downregulation of CXCR4/JAK2/SIRT5 signaling. EBioMedicine. 2019;42:64-75. doi:10.1016/j.ebiom.2019.03.031

249. Li ZK, Gao LF, Zhu XA, Xiang DK. LncRNA HOXA-AS3 Promotes the Progression of Pulmonary Arterial Hypertension through Mediation of miR-675-3p/PDE5A Axis. Biochem Genet. 2021;59(5):1158-1172. doi:10.1007/s10528-021-10053-y

250. Li H, Yan X, Yang M, Liu M, Tian S, Yu M, Li WP, Zhang C. The Impact of PTPRK and ROS1 Polymorphisms on the Preeclampsia Risk in Han Chinese Women. Int J Hypertens. 2021;2021:3275081. doi:10.1155/2021/3275081

251. Liu Y, Huang J, Yu N, Wei S, Liu Z. Involvement of WNT2 in trophoblast cell behavior in preeclampsia development. Cell Cycle. 2020;19(17):2207-2215. doi:10.1080/15384101.2020.1802913 
252. Ding L, Blitz MJ, Wing DA, Epstein AJ, Gjessing HK, Wilson ML. PHLDA2 gene polymorphisms and risk of HELLP syndrome and severe preeclampsia. Pregnancy Hypertens. 2020;19:190-194. doi:10.1016/j.preghy.2020.01.013

253. Li G, Wei W, Suo L, Zhang C, Yu H, Liu H, Guo Q, Zhen X, Yu Y. Low-Dose Aspirin Prevents Kidney Damage in LPS-Induced Preeclampsia by Inhibiting the WNT5A and NF- $\kappa$ B Signaling Pathways. Front Endocrinol (Lausanne). 2021;12:639592. doi:10.3389/fendo.2021.639592

254. Majchrzak-Celińska A, Kosicka K, Paczkowska J, Główka FK, Bręborowicz GH, Krzyścin M, Siemiątkowska A, Szaumkessel M, BaerDubowska W. HSD11B2, RUNX3, and LINE-1 Methylation in Placental DNA of Hypertensive Disorders of Pregnancy Patients. Reprod Sci. 2017;24(11):1520-1531. doi:10.1177/1933719117692043

255. Zhang YC, Qin XL, Ma XL, Mo HQ, Qin S, Zhang CX, Wei XW, Liu XQ, Zhang Y, Tian FJ, et al. CLDN1 regulates trophoblast apoptosis and proliferation in preeclampsia. Reproduction. 2021;161(6):623-632. doi:10.1530/REP-20-0677

256. Ma R, Gu Y, Zhao S, Sun J, Groome LJ, Wang Y. Expressions of vitamin D metabolic components VDBP, CYP2R1, CYP27B1, CYP24A1, and VDR in placentas from normal and preeclamptic pregnancies. Am J Physiol Endocrinol Metab. 2012;303(7):E928-E935. doi:10.1152/ajpendo.00279.2012

257. Zhou C, Song C, Huang X, Chen S, Long Y, Zeng S, Yang H, Jiang M. Early Prediction Model of Gestational Hypertension by MultiBiomarkers Before 20 Weeks Gestation. Diabetes Metab Syndr Obes. 2021;14:2441-2451. doi:10.2147/DMSO.S309725

258. Chen A, Li C, Wang J, Sha H, Piao S, Liu S. Role of Toll-Like Receptor 3 Gene Polymorphisms in Preeclampsia. Cell Physiol Biochem. 2015;37(5):1927-1933. doi:10.1159/000438553

259. Duan FM, Fu LJ, Wang YH, Adu-Gyamfi EA, Ruan LL, Xu ZW, Xiao SQ, Chen XM, Wang YX, Liu TH, et al. THBS1 regulates trophoblast fusion through a CD36-dependent inhibition of cAMP, and its upregulation participates in preeclampsia. Genes Dis. 2020;8(3):353-363. doi:10.1016/j.gendis.2020.05.007 
260. Yu Y, Zhang L, Xu G, Wu Z, Li Q, Gu Y, Niu J. Angiotensin II Type I Receptor Agonistic Autoantibody Induces Podocyte Injury via Activation of the TRPC6- Calcium/Calcineurin Pathway in Pre-Eclampsia. Kidney Blood Press Res. 2018;43(5):1666-1676. doi:10.1159/000494744

261. Purwosunu Y, Sekizawa A, Farina A, Wibowo N, Okazaki S, Nakamura M, Samura O, Fujito N, Okai T. Cell-free mRNA concentrations of CRH, PLAC1, and selectin-P are increased in the plasma of pregnant women with preeclampsia. Prenat Diagn. 2007;27(8):772-777. doi:10.1002/pd.1780

262. Zeng S, Pan Y, Liu F, Yin J, Jiang M, Long Y, Zhao X, Lash GE, Yang H. Role of clusterin in the regulation of trophoblast development and preeclampsia. Biochem Biophys Res Commun. 2021;583:128-134. doi:10.1016/j.bbrc.2021.10.064

263. Kreis NN, Friemel A, Jennewein L, Hoock SC, Hentrich AE, Nowak T, Louwen F, Yuan J. Functional Analysis of p21Cip1/CDKN1A and Its Family Members in Trophoblastic Cells of the Placenta and Its Roles in Preeclampsia. Cells. 2021;10(9):2214. doi:10.3390/cells10092214

264. Zou AX, Chen B, Li QX, Liang YC. MiR-134 inhibits infiltration of trophoblast cells in placenta of patients with preeclampsia by decreasing ITGB1 expression. Eur Rev Med Pharmacol Sci. 2018;22(8):2199-2206. doi:10.26355/eurrev_201804_14804

265. Nilsson LL, Djurisic S, Andersen AM, Melbye M, Bjerre D, FerreroMiliani L, Hackmon R, Geraghty DE, Hviid TV. Distribution of HLA-G extended haplotypes and one HLA-E polymorphism in a large-scale study of mother-child dyads with and without severe preeclampsia and eclampsia. HLA. 2016;88(4):172-186. doi:10.1111/tan.12871

266. Zhang Z, Gao Y, Zhang L, Jia L, Wang P, Zhang L, Li H. Alterations of IL-6, IL-6R and gp130 in early and late onset severe preeclampsia. Hypertens Pregnancy. 2013;32(3):270-280. doi:10.3109/10641955.2013.798332

267. Hu G, Xia ZS, Guo X. Differential expression of serum GBP-28, NBP-Cyc 3 and TIMP-1 complicates pregnancy in hypertensive disorder pregnancy. J Reprod Immunol. 2021;144:103288. doi:10.1016/j.jri.2021.103288 
268. Starodubtseva N, Nizyaeva N, Baev O, Bugrova A, Gapaeva M, Muminova K, Kononikhin A, Frankevich V, Nikolaev E, Sukhikh G. SERPINA1 Peptides in Urine as A Potential Marker of Preeclampsia Severity. Int J Mol Sci. 2020;21(3):914. doi:10.3390/ijms21030914

269. Przybyl L, Haase N, Golic M, Rugor J, Solano ME, Arck PC, Gauster M, Huppertz B, Emontzpohl C, Stoppe C, et al. CD74-Downregulation of Placental Macrophage-Trophoblastic Interactions in Preeclampsia. Circ Res. 2016;119(1):55-68. doi:10.1161/CIRCRESAHA.116.308304

270. Meems LM, Mahmud H, Buikema H, Tost J, Michel S, Takens J, Verkaik-Schakel RN, Vreeswijk-Baudoin I, Mateo-Leach IV, van der Harst $\mathrm{P}$, et al. Parental vitamin $\mathrm{D}$ deficiency during pregnancy is associated with increased blood pressure in offspring via Panx1 hypermethylation. Am J Physiol Heart Circ Physiol. 2016;311(6):H1459-H1469. doi:10.1152/ajpheart.00141.2016

271. Shinya K, Nakayama T, Nakayama T, Yamamoto T. A case-control study between the STIM1 gene and hypertensive disorders of pregnancy. Hypertens Res. 2018;41(1):39-44. doi:10.1038/hr.2017.84

272. Lei GQ, Wu ZY, Jiang WB, Luo J, Xu H, Luo SF, Peng ZY, Wang W, Chen M, Yu LL. Effect of CXCL12/CXCR4 on migration of deciduaderived mesenchymal stem cells from pregnancies with preeclampsia. Am J Reprod Immunol. 2019;82(5):e13180. doi:10.1111/aji.13180

273. Liu X, Lu L, Yao P, Ma Y, Wang F, Jin Q, Ye X, Li H, Hu FB, Sun $\mathrm{L}$, et al. Lipopolysaccharide binding protein, obesity status and incidence of metabolic syndrome: a prospective study among middle-aged and older Chinese. Diabetologia. 2014;57(9):1834-1841. doi:10.1007/s00125-0143288-7

274. Neocleous V, Shammas C, Phelan MM, Fanis P, Pantelidou M, Skordis N, Mantzoros C, Phylactou LA, Toumba M. A novel MC4R deletion coexisting with FTO and MC1R gene variants, causes severe early onset obesity. Hormones (Athens). 2016;15(3):445-452. doi:10.14310/horm.2002.1686

275. Cereijo R, Quesada-López T, Gavaldà-Navarro A, Tarascó J, Pellitero S, Reyes M, Puig-Domingo M, Giralt M, Sánchez-Infantes D, Villarroya F. The chemokine CXCL14 is negatively associated with obesity and 
concomitant type-2 diabetes in humans. Int J Obes (Lond). 2021;45(3):706710. doi:10.1038/s41366-020-00732-y

276. Yamaguchi M, Murata T. Involvement of regucalcin gene promoter region-related protein-p117, a transcription factor, in human obesity. Biomed Rep. 2017;6(4):374-378. doi:10.3892/br.2017.874

277. Lear S, Pflimlin E, Zhou Z, Huang D, Weng S, Nguyen-Tran V, Joseph SB, Roller S, Peterson S, Li J, et al. Engineering of a Potent, LongActing NPY2R Agonist for Combination with a GLP-1R Agonist as a MultiHormonal Treatment for Obesity. J Med Chem. 2020;63(17):9660-9671. doi:10.1021/acs.jmedchem.0c00740

278. Vaittinen M, Kolehmainen M, Rydén M, Eskelinen M, Wabitsch M, Pihlajamäki J, Uusitupa M, Pulkkinen L. et al. MFAP5 is related to obesityassociated adipose tissue and extracellular matrix remodeling and inflammation. Obesity (Silver Spring). 2015;23(7):1371-1378. doi:10.1002/oby.21103

279. Koutaki D, Michos A, Bacopoulou F, Charmandari E. The Emerging Role of Sfrp5 and Wnt5a in the Pathogenesis of Obesity: Implications for a Healthy Diet and Lifestyle. Nutrients. 2021;13(7):2459. doi:10.3390/nu13072459

280. Awazawa M, Gabel P, Tsaousidou E, Nolte H, Krüger M, Schmitz J, Ackermann PJ, Brandt C, Altmüller J, Motameny S, et al. A microRNA screen reveals that elevated hepatic ectodysplasin A expression contributes to obesity-induced insulin resistance in skeletal muscle. Nat Med. 2017;23(12):1466-1473. doi:10.1038/nm.4420

281. Ahmad M, Nongmaithem SS, Krishnaveni GV, Fall CH, Yajnik CS, Chandak GR. Lack of replication of association of THSD7A with obesity. Int J Obes (Lond). 2016;40(4):725-726. doi:10.1038/ijo.2016.15

282. Gonsorcíková L, Průhová S, Cinek O, Ek J, Pelikánová T, Jørgensen T, Eiberg H, Pedersen O, Hansen T, Lebl J. Autosomal inheritance of diabetes in two families characterized by obesity and a novel H241Q mutation in NEUROD1. Pediatr Diabetes. 2008;9(4 Pt 2):367-372. doi:10.1111/j.1399-5448.2008.00379.x

283. Lim R, Lappas M. Slit2 exerts anti-inflammatory actions in human placenta and is decreased with maternal obesity. Am J Reprod Immunol. 2015;73(1):66-78. doi:10.1111/aji.12334 
284. Davies M, Fraser SA, Galic S, Choy SW, Katerelos M, Gleich K, Kemp BE, Mount PF, Power DA. Novel mechanisms of $\mathrm{Na}+$ retention in obesity: phosphorylation of NKCC2 and regulation of SPAK/OSR1 by AMPK. Am J Physiol Renal Physiol. 2014;307(1):F96-F106. doi:10.1152/ajprenal.00524.2013

285. Fabre O, Breuker C, Amouzou C, Salehzada T, Kitzmann M, Mercier J, Bisbal C. Defects in TLR3 expression and RNase L activation lead to decreased MnSOD expression and insulin resistance in muscle cells of obese people. Cell Death Dis. 2014;5(3):e1136. doi:10.1038/cddis.2014.104

286. Boon MR, van den Berg SA, Wang Y, van den Bossche J, Karkampouna S, Bauwens M, De Saint-Hubert M, van der Horst G, Vukicevic S, de Winther MP, et al. BMP7 activates brown adipose tissue and reduces diet-induced obesity only at subthermoneutrality. PLoS One. 2013;8(9):e74083. doi:10.1371/journal.pone.0074083

287. Yang Y, Zhang Y, Zhou X, Chen D, Ouyang G, Liu Y, Cui D. Periostin deficiency attenuates lipopolysaccharide- and obesity-induced adipose tissue fibrosis. FEBS Lett. 2021;595(16):2099-2112. doi:10.1002/1873-3468.14154

288. Lee S. The genetic and epigenetic association of LDL Receptor Related Protein 1B (LRP1B) gene with childhood obesity. Sci Rep. 2019;9(1):1815. Published 2019 Feb 12. doi:10.1038/s41598-019-38538-2

289. Matsuo Y, Tanaka M, Yamakage H, Sasaki Y, Muranaka K, Hata H, Ikai I, Shimatsu A, Inoue M, Chun TH, et al. Thrombospondin 1 as a novel biological marker of obesity and metabolic syndrome. Metabolism. 2015;64(11):1490-1499. doi:10.1016/j.metabol.2015.07.016

290. Grarup N, Andersen G, Krarup NT, Albrechtsen A, Schmitz O, Jørgensen T, Borch-Johnsen K, Hansen T, Pedersen O. Association testing of novel type 2 diabetes risk alleles in the JAZF1, CDC123/CAMK1D, TSPAN8, THADA, ADAMTS9, and NOTCH2 loci with insulin release, insulin sensitivity, and obesity in a population-based sample of 4,516 glucose-tolerant middle-aged Danes. Diabetes. 2008;57(9):2534-2540. doi:10.2337/db08-0436

291. Masson O, Chavey C, Dray C, Meulle A, Daviaud D, Quilliot D, Muller C, Valet P, Liaudet-Coopman E. LRP1 receptor controls 
adipogenesis and is up-regulated in human and mouse obese adipose tissue.

PLoS One. 2009;4(10):e7422. doi:10.1371/journal.pone.0007422.

292. Park JS, Lee WK, Kim HS, Seo JA, Kim DH, Han HC, Min BH. C

Clusterin overexpression protects against western diet-induced obesity and

NAFLD. Sci Rep. 2020;10(1):17484. doi:10.1038/s41598-020-73927-y

293. Collet C, Laplanche JL, de Vernejoul MC. Camurati-Engelmann disease with obesity in a newly identified family carrying a missense p.Arg156Cys mutation in the TGFB1 gene. Am J Med Genet A. 2013;161A(8):2074-2077. doi:10.1002/ajmg.a.36022

294. An YA, Crewe C, Asterholm IW, Sun K, Chen S, Zhang F, Shao M, Funcke JB, Zhang Z, Straub L, et al. Dysregulation of Amyloid Precursor Protein Impairs Adipose Tissue Mitochondrial Function and Promotes Obesity. Nat Metab. 2019;1(12):1243-1257. doi:10.1038/s42255-019-01491

295. Awaya T, Yokosaki Y, Yamane K, Usui H, Kohno N, Eboshida A. Gene-environment association of an ITGB2 sequence variant with obesity in ethnic Japanese. Obesity (Silver Spring). 2008;16(6):1463-1466. doi:10.1038/oby.2008.68

296. Sindhu S, Thomas R, Shihab P, Sriraman D, Behbehani K, Ahmad R. Obesity Is a Positive Modulator of IL-6R and IL-6 Expression in the Subcutaneous Adipose Tissue: Significance for Metabolic Inflammation. PLoS One. 2015;10(7):e0133494. doi:10.1371/journal.pone.0133494

297. Andrade VL, Petruceli E, Belo VA, Andrade-Fernandes CM, Caetano Russi CV, Bosco AA, Tanus-Santos JE, Sandrim VC. Evaluation of plasmatic MMP-8, MMP-9, TIMP-1 and MPO levels in obese and lean women. Clin Biochem. 2012;45(6):412-415. doi:10.1016/j.clinbiochem.2012.01.008

298. Li D, Gwag T, Wang S. Absence of CD47 maintains brown fat thermogenic capacity and protects mice from aging-related obesity and metabolic disorder. Biochem Biophys Res Commun. 2021;575:14-19. doi:10.1016/j.bbrc.2021.08.062

299. Chan PC, Wu TN, Chen YC, Lu CH, Wabitsch M, Tian YF, Hsieh PS. Targetted inhibition of CD74 attenuates adipose COX-2-MIF-mediated M1 macrophage polarization and retards obesity-related adipose tissue 
inflammation and insulin resistance. Clin Sci (Lond). 2018;132(14):15811596. doi:10.1042/CS20180041

300. Redonnet A, Bonilla S, Noël-Suberville C, Pallet V, Dabadie H, Gin

H, Higueret P. Relationship between peroxisome proliferator-activated receptor gamma and retinoic acid receptor alpha gene expression in obese human adipose tissue. Int J Obes Relat Metab Disord. 2002;26(7):920-927. doi:10.1038/sj.ijo.0802025

301. Qian G, Adeyanju O, Sunil C, Huang SK, Chen SY, Tucker TA, Idell S, Guo X. Dedicator of cytokinesis 2 (DOCK2) deficiency attenuates lung injury associated with chronic high-fat and high-fructose diet-induced obesity. Am J Pathol. 2021;S0002-9440(21)00474-0. doi:10.1016/j.ajpath.2021.10.011

302. Kaartinen MT, Arora M, Heinonen S, Rissanen A, Kaprio J, Pietiläinen KH. Transglutaminases and Obesity in Humans: Association of F13A1 to Adipocyte Hypertrophy and Adipose Tissue Immune Response. Int J Mol Sci. 2020;21(21):8289. doi:10.3390/ijms21218289

303. Kuroda M, Nishiguchi M, Ugawa N, Ishikawa E, Kawabata Y, Okamoto S, Sasaki W, Miyatake Y, Sebe M, Masumoto S, et al. Interferon regulatory factor 7 mediates obesity-associated MCP-1 transcription. PLoS One. 2020;15(5):e0233390. doi:10.1371/journal.pone.0233390

304. Chen Z, Pan S, Yin K, Zhang Y, Yuan X, Wang S, Yang S, Shen Q, Tang Y, Li J, et al. Deficiency of ER Ca2+ sensor STIM1 in AgRP neurons confers protection against dietary obesity. Cell Rep. 2021;37(3):109868. doi:10.1016/j.celrep.2021.109868

305. Yao L, Heuser-Baker J, Herlea-Pana O, Zhang N, Szweda LI, Griffin TM, Barlic-Dicen J. Deficiency in adipocyte chemokine receptor CXCR4 exacerbates obesity and compromises thermoregulatory responses of brown adipose tissue in a mouse model of diet-induced obesity. FASEB J. 2014;28(10):4534-4550. doi:10.1096/fj.14-249797

306. Yoshida K, Kita Y, Tokuoka SM, Hamano F, Yamazaki M, Sakimura K, Kano M, Shimizu T. Monoacylglycerol lipase deficiency affects dietinduced obesity, fat absorption, and feeding behavior in CB1 cannabinoid receptor-deficient mice. FASEB J. 2019;33(2):2484-2497. doi:10.1096/fj.201801203R 
307. Jeyaratnaganthan N, Højlund K, Kroustrup JP, Larsen JF, Bjerre M, Levin K, Beck-Nielsen H, Frago S, Hassan AB, Flyvbjerg A, et al. Circulating levels of insulin-like growth factor-II/mannose-6-phosphate receptor in obesity and type 2 diabetes. Growth Horm IGF Res. 2010;20(3):185-191. doi:10.1016/j.ghir.2009.12.005

308. Kim TH, Yang YM, Han CY, Koo JH, Oh H, Kim SS, You BH, Choi YH, Park TS, Lee $\mathrm{CH}$, et al. Ga12 ablation exacerbates liver steatosis and obesity by suppressing USP22/SIRT1-regulated mitochondrial respiration. J Clin Invest. 2018;128(12):5587-5602. doi:10.1172/JCI97831

309. Wilson CH, Nikolic A, Kentish SJ, Keller M, Hatzinikolas G, Dorstyn L, Page AJ, Kumar S. Caspase-2 deficiency enhances whole-body carbohydrate utilisation and prevents high-fat diet-induced obesity. Cell Death Dis. 2017;8(10):e3136. doi:10.1038/cddis.2017.518

310. Foghsgaard S, Vedtofte L, Mathiesen ER, Svare JA, Gluud LL, Holst JJ, Damm P, Knop FK, Vilsbøll T. The effect of a glucagon-like peptide-1 receptor agonist on glucose tolerance in women with previous gestational diabetes mellitus: protocol for an investigator-initiated, randomised, placebo-controlled, double-blinded, parallel intervention trial. BMJ Open. 2013;3(10):e003834. doi:10.1136/bmjopen-2013-003834

311. Sagen JV, Baumann ME, Salvesen HB, Molven A, Søvik O, Njølstad PR. Diagnostic screening of NEUROD1 (MODY6) in subjects with MODY or gestational diabetes mellitus. Diabet Med. 2005;22(8):1012-1015. doi:10.1111/j.1464-5491.2005.01565.x

312. Kelstrup L, Hjort L, Houshmand-Oeregaard A, Clausen TD, Hansen NS, Broholm C, Borch-Johnsen L, Mathiesen ER, Vaag AA, Damm P. Gene Expression and DNA Methylation of PPARGC1A in Muscle and Adipose Tissue From Adult Offspring of Women With Diabetes in Pregnancy. Diabetes. 2016;65(10):2900-2910. doi:10.2337/db16-0227

313. Balachandiran M, Bobby Z, Dorairajan G, Gladwin V, Vinayagam V, Packirisamy RM. Decreased maternal serum adiponectin and increased insulin-like growth factor-1 levels along with increased placental glucose transporter-1 expression in gestational diabetes mellitus: Possible role in fetal overgrowth. Placenta. 2021;104:71-80. doi:10.1016/j.placenta.2020.11.008 
314. Houde AA, Ruchat SM, Allard C, Baillargeon JP, St-Pierre J, Perron P, Gaudet D, Brisson D, Hivert MF, Bouchard L. LRP1B, BRD2 and CACNA1D: new candidate genes in fetal metabolic programming of newborns exposed to maternal hyperglycemia. Epigenomics. 2015;7(7):1111-1122. doi:10.2217/epi.15.72

315. Tarnowski M, Malinowski D, Safranow K, Dziedziejko V, Pawlik A. HNF1B, TSPAN8 and NOTCH2 gene polymorphisms in women with gestational diabetes. J Matern Fetal Neonatal Med. 2018;31(7):837-842. doi:10.1080/14767058.2017.1297793

316. Li Y, Zhuang J. miR-345-3p serves a protective role during gestational diabetes mellitus by targeting BAK1. Exp Ther Med. 2021;21(1):2. doi:10.3892/etm.2020.9434

317. Li Q, Pereira TJ, Moyce BL, Mahood TH, Doucette CA, Rempel J, Dolinsky VW. In utero exposure to gestational diabetes mellitus conditions TLR4 and TLR2 activated IL-1beta responses in spleen cells from rat offspring. Biochim Biophys Acta. 2016;1862(11):2137-2146. doi:10.1016/j.bbadis.2016.08.004

318. Kuźmicki M, Telejko B, Lipińska D, Pliszka J, Wilk J, Wawrusiewicz-Kurylonek N, Zielińska A, Sobota A, Krętowski A, Górska $\mathrm{M}$, et al. The IL-6/IL-6R/sgp130 system and Th17 associated cytokines in patients with gestational diabetes. Endokrynol Pol. 2014;65(3):169-175. doi:10.5603/EP.2014.0023

319. Vilmi-Kerälä T, Lauhio A, Tervahartiala T, Palomäki O, Uotila J, Sorsa T, Palomäki A. Subclinical inflammation associated with prolonged TIMP-1 upregulation and arterial stiffness after gestational diabetes mellitus: a hospital-based cohort study. Cardiovasc Diabetol. 2017;16(1):49. doi:10.1186/s12933-017-0530-x

320. Wójcik M, Mac-Marcjanek K, Woźniak LA, Nadel I, Lewiński A, Cypryk K. The association of leukocyte phosphatidylinositol 3-kinase delta overexpression with gestational diabetes mellitus (GDM). Endokrynol Pol. 2014;65(1):17-24. doi:10.5603/EP.2014.0003

321. Wang F, Xu C, Reece EA, Li X, Wu Y, Harman C, Yu J, Dong D, Wang $\mathrm{C}$, Yang $\mathrm{P}$, et al. Protein kinase $\mathrm{C}$-alpha suppresses autophagy and induces neural tube defects via miR-129-2 in diabetic pregnancy. Nat Commun. 2017;8:15182. doi:10.1038/ncomms15182 
322. Zhang H, Luan S, Xiao X, Lin L, Zhao X, Liu X. Silenced microRNA-222 suppresses inflammatory response in gestational diabetes mellitus mice by promoting CXCR4. Life Sci. 2021;266:118850. doi:10.1016/j.lfs.2020.118850

323. Zhang L, Li K, Tian S, Wang XQ, Li JH, Dong YC, Xia HF, Ma X. Down-regulation of microRNA-30d-5p is associated with gestational diabetes mellitus by targeting RAB8A. J Diabetes Complications. 2021;35(8):107959. doi:10.1016/j.jdiacomp.2021.107959

324. Gelato MC, Rutherford C, San-Roman G, Shmoys S, Monheit A. The serum insulin-like growth factor-II/mannose-6-phosphate receptor in normal and diabetic pregnancy. Metabolism. 1993;42(8):1031-1038. doi:10.1016/0026-0495(93)90019-k

325. Kniotek M, Roszczyk A, Zych M, Wrzosek M, Szafarowska M, Zagożdżon R, Jerzak M. Sildenafil Citrate Downregulates PDE5A mRNA Expression in Women with Recurrent Pregnancy Loss without Altering Angiogenic Factors-A Preliminary Study. J Clin Med. 2021;10(21):5086. doi:10.3390/jcm10215086

326. Yang ZM, Chen LH, Hong M, Chen YY, Yang XR, Tang SM, Yuan QF, Chen WW. Serum microRNA profiling and bioinformatics analysis of patients with type 2 diabetes mellitus in a Chinese population. Mol Med Rep. 2017;15(4):2143-2153. doi:10.3892/mmr.2017.6239

327. Singh R, Ha SE, Wei L, Jin B, Zogg H, Poudrier SM, Jorgensen BG,

Park C, Ronkon CF, Bartlett A, et al. miR-10b-5p Rescues Diabetes and Gastrointestinal Dysmotility. Gastroenterology. 2021;160(5):1662-1678.e18. doi:10.1053/j.gastro.2020.12.062

328. Lv K, Guo Y, Zhang Y, Wang K, Jia Y, Sun S. Allele-specific targeting of hsa-miR-657 to human IGF2R creates a potential mechanism underlying the association of ACAA-insertion/deletion polymorphism with type 2 diabetes. Biochem Biophys Res Commun. 2008;374(1):101-105. doi:10.1016/j.bbrc.2008.06.102

329. Ding L, Cai M, Chen L, Yan H, Lu S, Pang S, Yan B. Identification and functional study of GATA4 gene regulatory variants in type 2 diabetes mellitus. BMC Endocr Disord. 2021;21(1):73. doi:10.1186/s12902-02100739-0 
330. Fogarty MP, Cannon ME, Vadlamudi S, Gaulton KJ, Mohlke KL. Identification of a regulatory variant that binds FOXA1 and FOXA2 at the CDC123/CAMK1D type 2 diabetes GWAS locus. PLoS Genet. 2014;10(9):e1004633. doi:10.1371/journal.pgen.1004633

331. Bi C, Fu Y, Li B. Brain-derived neurotrophic factor alleviates diabetes mellitus-accelerated atherosclerosis by promoting M2 polarization of macrophages through repressing the STAT3 pathway. Cell Signal. 2020;70:109569. doi:10.1016/j.cellsig.2020.109569

332. Zmuda EJ, Qi L, Zhu MX, Mirmira RG, Montminy MR, Hai T. The roles of ATF3, an adaptive-response gene, in high-fat-diet-induced diabetes and pancreatic beta-cell dysfunction. Mol Endocrinol. 2010;24(7):14231433. doi:10.1210/me.2009-0463

333. Yang Z, Wei Z, Wu X, Yang H. Screening of exosomal miRNAs derived from subcutaneous and visceral adipose tissues: Determination of targets for the treatment of obesity and associated metabolic disorders. Mol Med Rep. 2018;18(3):3314-3324. doi:10.3892/mmr.2018.9312

334. Wang P, Wang Z, Liu G, Jin C, Zhang Q, Man S, Wang Z. miR-657 Promotes Macrophage Polarization toward M1 by Targeting FAM46C in Gestational Diabetes Mellitus. Mediators Inflamm. 2019;2019:4851214. doi: $10.1155 / 2019 / 4851214$

335. Improta Caria AC, Nonaka CKV, Pereira CS, Soares MBP, Macambira SG, Souza BSF. Exercise Training-Induced Changes in MicroRNAs: Beneficial Regulatory Effects in Hypertension, Type 2 Diabetes, and Obesity. Int J Mol Sci. 2018;19(11):3608. Published 2018 Nov 15. doi:10.3390/ijms 19113608

336. Li K, Li Y, Yu Y, Ding J, Huang H, Chu C, Hu L, Yu Y, Cao Y, Xu $\mathrm{P}$, et al. Bmi-1 alleviates adventitial fibroblast senescence by eliminating ROS in pulmonary hypertension. BMC Pulm Med. 2021;21(1):80. doi:10.1186/s12890-021-01439-0

337. Park AM, Wong CM, Jelinkova L, Liu L, Nagase H, Suzuki YJ. Pulmonary hypertension-induced GATA4 activation in the right ventricle. Hypertension. 2010;56(6):1145-1151. doi:10.1161/HYPERTENSIONAHA.110.160515

338. Cheng G, He L, Zhang Y. LincRNA-Cox2 promotes pulmonary arterial hypertension by regulating the let-7a-mediated STAT3 signaling 
pathway. Mol Cell Biochem. 2020;475(1-2):239-247. doi:10.1007/s11010020-03877-6

339. Davis J. Putting the Brakes on Hypertensive Remodeling: An ATF3

Mechanism of Myofibroblast Restraint. Circulation. 2017;135(21):20582061. doi:10.1161/CIRCULATIONAHA.117.028020

340. Patankar JV, Chandak PG, Obrowsky S, Pfeifer T, Diwoky C, Uellen A, Sattler W, Stollberger R, Hoefler G, Heinemann A, et al. Loss of intestinal GATA4 prevents diet-induced obesity and promotes insulin sensitivity in mice. Am J Physiol Endocrinol Metab. 2011;300(3):E478E488. doi:10.1152/ajpendo.00457.2010

341. Bochkis IM, Shin S, Kaestner KH. Bile acid-induced inflammatory signaling in mice lacking Foxa2 in the liver leads to activation of mTOR and age-onset Mosity. Metab. 2013;2(4):447-456. doi:10.1016/j.molmet.2013.08.005

342. Nishimura Y, Sasagawa S, Ariyoshi M, Ichikawa S, Shimada Y, Kawaguchi K, Kawase R, Yamamoto R, Uehara T, Yanai T, et al. Systems pharmacology of adiposity reveals inhibition of EP300 as a common therapeutic mechanism of caloric restriction and resveratrol for obesity. Front Pharmacol. 2015;6:199. doi:10.3389/fphar.2015.00199

343. Su T, Huang C, Yang C, Jiang T, Su J, Chen M, Fatima S, Gong R, $\mathrm{Hu} \mathrm{X}$, Bian Z, et al. Apigenin inhibits STAT3/CD36 signaling axis and reduces visceral obesity. Pharmacol Res. 2020;152:104586. doi:10.1016/j.phrs.2019.104586

344. Kim S, Song NJ, Bahn G, Chang SH, Yun UJ, Ku JM, Jo DG, Park $\mathrm{KW}$. Atf3 induction is a therapeutic target for obesity and metabolic diseases. Biochem Biophys Res Commun. 2018;504(4):903-908. doi:10.1016/j.bbrc.2018.09.048

345. Liang J, Bai S, Su L, Li C, Wu J, Xia Z, Xu D. A subset of circulating microRNAs is expressed differently in patients with myocardial infarction. Mol Med Rep. 2015;12(1):243-247. doi:10.3892/mmr.2015.3422

346. Zhang J, Wu L, Li Z, Fu G. miR-1231 exacerbates arrhythmia by targeting calciumchannel gene CACNA2D2 in myocardial infarction. Am J Transl Res. 2017;9(4):1822-1833.

347. Schulte JS, Fehrmann E, Tekook MA, Kranick D, Fels B, Li N, Wehrens XH, Heinick A, Seidl MD, Schmitz W, et al. Cardiac expression of 
the CREM repressor isoform CREM-Ib $\triangle \mathrm{C}-\mathrm{X}$ in mice leads to arrhythmogenic alterations in ventricular cardiomyocytes. Basic Res Cardiol. 2016;111(2):15. doi:10.1007/s00395-016-0532-y

348. Yang W, Wu Z, Yang K, Han Y, Chen Y, Zhao W, Huang F, Jin Y, Jin W. BMI1 promotes cardiac fibrosis in ischemia-induced heart failure via the PTEN-PI3K/Akt-mTOR signaling pathway. Am J Physiol Heart Circ Physiol. 2019;316(1):H61-H69. doi:10.1152/ajpheart.00487.2018

349. Liu Y, Wang Z, Xiao W. MicroRNA-26a protects against cardiac hypertrophy via inhibiting GATA4 in rat model and cultured cardiomyocytes. Mol Med Rep. 2016;14(3):2860-2866. doi:10.3892/mmr.2016.5574

350. Rouhi L, Fan S, Cheedipudi SM, Braza-Boïls A, Molina MS, Yao Y, Robertson MJ, Coarfa C, Gimeno JR, Molina P, et al. The EP300/TP53 pathway, a suppressor of the Hippo and canonical WNT pathways, is activated in human hearts with arrhythmogenic cardiomyopathy in the absence of overt heart failure. Cardiovasc Res. 2021;cvab197. doi:10.1093/cvr/cvab197

351. Bao Q, Zhang B, Suo Y, Liu C, Yang Q, Zhang K, Yuan M, Yuan M, Zhang Y, Li G. Intermittent hypoxia mediated by TSP1 dependent on STAT3 induces cardiac fibroblast activation and cardiac fibrosis. Elife. 2020;9:e49923. doi:10.7554/eLife.49923

352. Liu XM, Du SL, Miao R, Wang LF, Zhong JC. Targeting the forkhead box protein P1 pathway as a novel therapeutic approach for cardiovascular diseases. Heart Fail Rev. 2020;10.1007/s10741-020-09992-2. doi:10.1007/s10741-020-09992-2

353. Zhou H, Li N, Yuan Y, Jin YG, Guo H, Deng W, Tang QZ. Activating transcription factor 3 in cardiovascular diseases: a potential therapeutic target. Basic Res Cardiol. 2018;113(5):37. doi:10.1007/s00395018-0698-6

354. Gao Y, Tang Y, Sun Q, Guan G, Wu X, Shi F, Zhou Z, Yang W. Circular RNA FOXP1 relieves trophoblastic cell dysfunction in recurrent pregnancy loss via the miR-143-3p/S100A11 cascade. Bioengineered. 2021;12(1):9081-9093. doi:10.1080/21655979.2021.1988374 


\section{Tables}

Table 1 The statistical metrics for key differentially expressed genes (DEGs)

\begin{tabular}{|c|c|c|c|c|c|c|}
\hline GeneSymbol & $\log \mathrm{FC}$ & pValue & adj.P.Val & tvalue & Up/Down & GeneName \\
\hline OPN1LW & 5.513445 & $1.08 \mathrm{E}-13$ & $1.33 \mathrm{E}-09$ & 7.430209 & $\mathrm{Up}$ & opsin 1 , long wave sensitive \\
\hline NDP & 4.18255 & $8.43 \mathrm{E}-05$ & 0.006062 & 3.93188 & Up & norrin cystine knot growth factor NDP \\
\hline CXorf66 & 4.046972 & 0.000314 & 0.013896 & 3.603447 & Up & chromosome $\mathrm{X}$ open reading frame 66 \\
\hline TMPRSS11B & 3.991258 & 0.000207 & 0.011081 & 3.709816 & Up & transmembrane serine protease $11 \mathrm{~B}$ \\
\hline LINC02008 & 3.938406 & $2.43 \mathrm{E}-05$ & 0.003077 & 4.22139 & Up & long intergenic non-protein coding RNA 2008 \\
\hline LINC01697 & 3.891591 & 0.000675 & 0.022111 & 3.399696 & Up & long intergenic non-protein coding RNA 1697 \\
\hline HNF4G & 3.886017 & $3.11 \mathrm{E}-05$ & 0.003434 & 4.165549 & Up & hepatocyte nuclear factor 4 gamma \\
\hline HTN3 & 3.855467 & 0.000722 & 0.022988 & 3.381116 & Up & histatin 3 \\
\hline MTND5P28 & 3.791132 & 0.000284 & 0.01323 & 3.629045 & Up & MT-ND5 pseudogene 28 \\
\hline LINC02658 & 3.749396 & 0.000275 & 0.012942 & 3.638237 & Up & long intergenic non-protein coding RNA 2658 \\
\hline SLC15A1 & 3.747731 & $9.18 \mathrm{E}-06$ & 0.001667 & 4.43569 & Up & solute carrier family 15 member 1 \\
\hline ADAM18 & 3.744252 & 0.000249 & 0.012032 & 3.663474 & Up & ADAM metallopeptidase domain 18 \\
\hline LGALS17A & 3.665543 & 0.000865 & 0.024898 & 3.331024 & Up & galectin 14 pseudogene \\
\hline MTND6P20 & 3.650359 & 0.000196 & 0.010678 & 3.724129 & Up & MT-ND6 pseudogene 20 \\
\hline NPHP3-AS1 & 3.614647 & 0.000181 & 0.010146 & 3.743861 & Up & NPHP3 antisense RNA 1 \\
\hline SERPINA10 & 3.598318 & 0.001245 & 0.030213 & 3.228435 & Up & serpin family A member 10 \\
\hline C12orf54 & 3.590508 & 0.00037 & 0.015208 & 3.560355 & Up & chromosome 12 open reading frame 54 \\
\hline SPATA22 & 3.536618 & $1.95 \mathrm{E}-06$ & 0.00063 & 4.758353 & Up & spermatogenesis associated 22 \\
\hline LGR5 & 3.535902 & 0.000243 & 0.011968 & 3.669005 & Up & leucine rich repeat containing $\mathrm{G}$ protein-coupled receptor 5 \\
\hline RNU6-83P & 3.532468 & 0.001496 & 0.03347 & 3.1754 & Up & RNA, U6 small nuclear 83, pseudogene \\
\hline OCA2 & 3.531677 & 0.000113 & 0.00729 & 3.860849 & Up & OCA2 melanosomal transmembrane protein \\
\hline CHEK2P2 & 3.523224 & 0.001579 & 0.034406 & 3.159792 & Up & checkpoint kinase 2 pseudogene 2 \\
\hline PHYHIPL & 3.510438 & $1.05 \mathrm{E}-06$ & 0.00041 & 4.881476 & Up & phytanoyl-CoA 2-hydroxylase interacting protein like \\
\hline MTND5P17 & 3.509858 & 0.001127 & 0.028774 & 3.256804 & Up & MT-ND5 pseudogene 17 \\
\hline MTND1P6 & 3.503479 & 0.002651 & 0.045307 & 3.005581 & Up & MT-ND1 pseudogene 6 \\
\hline PLSCR2 & 3.501271 & 0.00175 & 0.036613 & 3.129626 & Up & phospholipid scramblase 2 \\
\hline OR1H1P & 3.500832 & 0.001555 & 0.034141 & 3.164149 & Up & olfactory receptor family 1 subfamily $\mathrm{H}$ member 1 pseudogene \\
\hline MIMT1 & 3.496863 & 0.001008 & 0.02697 & 3.288372 & Up & MER 1 repeat containing imprinted transcript 1 \\
\hline C3orf22 & 3.485741 & 0.000676 & 0.022122 & 3.39919 & Up & chromosome 3 open reading frame 22 \\
\hline $\mathrm{CDH} 3$ & 3.480793 & 4.35E-05 & 0.00417 & 4.087837 & Up & cadherin 3 \\
\hline LOC101927359 & 3.476698 & 0.000107 & 0.007035 & 3.874707 & Up & uncharacterized LOC101927359 \\
\hline MTND5P23 & 3.472296 & 0.001424 & 0.032562 & 3.189774 & Up & MT-ND5 pseudogene 23 \\
\hline LINC00906 & 3.468795 & 0.000953 & 0.026372 & 3.304029 & Up & long intergenic non-protein coding RNA 906 \\
\hline LIPJ & 3.468169 & 0.000369 & 0.015169 & 3.561489 & Up & lipase family member $\mathrm{J}$ \\
\hline NLRP8 & 3.44517 & 0.000862 & 0.02485 & 3.332101 & Up & NLR family pyrin domain containing 8 \\
\hline СВX3Р8 & 3.445133 & 0.000223 & 0.011379 & 3.690871 & Up & chromobox 3 pseudogene 8 \\
\hline PAEP & 3.437944 & 0.002141 & 0.0409 & 3.069962 & Up & progestagen associated endometrial protein \\
\hline RNY4P7 & 3.430056 & 0.000337 & 0.014383 & 3.585244 & Up & RNY4 pseudogene 7 \\
\hline
\end{tabular}




\begin{tabular}{|c|c|c|c|c|c|c|}
\hline KCNH7-AS1 & 3.427803 & 0.001973 & 0.03916 & 3.094316 & Up & KCNH7 antisense RNA 1 \\
\hline SIX1 & 3.427656 & $2.21 \mathrm{E}-05$ & 0.002943 & 4.243042 & Up & SIX homeobox 1 \\
\hline CUBNP3 & 3.421282 & 0.00223 & 0.041454 & 3.057715 & Up & cubilin pseudogene 3 \\
\hline CYP27C1 & 3.414289 & $3.25 \mathrm{E}-05$ & 0.003516 & 4.155216 & Up & cytochrome P450 family 27 subfamily C member 1 \\
\hline SLC34A2 & 3.403709 & $1.04 \mathrm{E}-05$ & 0.001765 & 4.407902 & Up & solute carrier family 34 member 2 \\
\hline DSG4 & 3.40126 & 0.000746 & 0.023321 & 3.372169 & Up & desmoglein 4 \\
\hline ASPA & 3.395598 & 4.34E-06 & 0.001071 & 4.594369 & Up & aspartoacylase \\
\hline SPATA16 & 3.379004 & 0.00073 & 0.023055 & 3.378147 & Up & spermatogenesis associated 16 \\
\hline CPN2 & 3.377474 & 0.000702 & 0.022707 & 3.388776 & Up & carboxypeptidase $\mathrm{N}$ subunit 2 \\
\hline SFTA3 & 3.361222 & 0.00085 & 0.024662 & 3.336103 & Up & surfactant associated 3 \\
\hline C8orf86 & 3.347714 & 0.000214 & 0.011264 & 3.702057 & Up & chromosome 8 open reading frame 86 \\
\hline GREM1 & 3.342215 & 0.000634 & 0.02135 & 3.416659 & Up & gremlin 1 , DAN family BMP antagonist \\
\hline MAGEA10 & 3.339161 & 0.000308 & 0.013776 & 3.608067 & Up & MAGE family member A10 \\
\hline LRCOL1 & 3.336085 & 0.001761 & 0.036748 & 3.127794 & Up & leucine rich colipase like 1 \\
\hline DDX18P2 & 3.334146 & 0.000979 & 0.026694 & 3.296526 & Up & DEAD-box helicase 18 pseudogene 2 \\
\hline LOC100129935 & 3.323216 & 0.002591 & 0.044767 & 3.012492 & Up & galectin 14 pseudogene \\
\hline LRRN4CL & 3.322787 & 0.000364 & 0.015029 & 3.565236 & Up & LRRN4 C-terminal like \\
\hline RAD51AP2 & 3.321826 & 0.002858 & 0.047155 & 2.982626 & Up & RAD51 associated protein 2 \\
\hline ANKRD62 & 3.311208 & 0.000281 & 0.013123 & 3.63219 & Up & ankyrin repeat domain 62 \\
\hline TXNDC8 & 3.305393 & 0.001998 & 0.039496 & 3.090579 & Up & thioredoxin domain containing 8 \\
\hline KLHL31 & 3.298714 & 0.000873 & 0.025053 & 3.328638 & Up & kelch like family member 31 \\
\hline GLRA3 & 3.290448 & 2.67E-06 & 0.000788 & 4.694779 & Up & glycine receptor alpha 3 \\
\hline PCDHGB6 & 3.281157 & 0.000288 & 0.013293 & 3.626107 & Up & protocadherin gamma subfamily $\mathrm{B}, 6$ \\
\hline MTND5P30 & 3.279389 & 0.001001 & 0.026856 & 3.290179 & Up & MT-ND5 pseudogene 30 \\
\hline MTCO1P22 & 3.276334 & 0.000294 & 0.013396 & 3.620554 & Up & MT-CO1 pseudogene 22 \\
\hline NEUROD4 & 3.271933 & 0.000648 & 0.021519 & 3.410775 & Up & neuronal differentiation 4 \\
\hline RGS5-AS1 & 3.271195 & 0.000323 & 0.014101 & 3.595763 & Up & RGS5 antisense RNA 1 \\
\hline CRISP2 & 3.262188 & 0.000128 & 0.007983 & 3.829692 & Up & cysteine rich secretory protein 2 \\
\hline FAM205C & 3.261825 & 0.001293 & 0.030853 & 3.217507 & Up & family with sequence similarity 205 member C \\
\hline GAS1RR & 3.261656 & 0.002178 & 0.041176 & 3.06484 & Up & GAS1 adjacent regulatory RNA \\
\hline PLCE1-AS2 & 3.257854 & 0.003008 & 0.048538 & 2.966893 & Up & PLCE1 antisense RNA 2 \\
\hline $\mathrm{CCDC} 160$ & 3.25178 & 0.002345 & 0.042557 & 3.042632 & Up & coiled-coil domain containing 160 \\
\hline FAM83B & 3.229445 & 0.002217 & 0.041334 & 3.059495 & Up & family with sequence similarity 83 member B \\
\hline ART3 & 3.227592 & 0.002995 & 0.048449 & 2.968252 & Up & ADP-ribosyltransferase 3 (inactive) \\
\hline SNAP25-AS1 & 3.223636 & 0.000944 & 0.026225 & 3.306718 & Up & SNAP25 antisense RNA 1 \\
\hline LINC02179 & 3.219018 & 0.000283 & 0.013182 & 3.630541 & Up & long intergenic non-protein coding RNA 2179 \\
\hline FAM204CP & 3.217918 & 0.003044 & 0.048837 & 2.963272 & Up & family with sequence similarity 204 member C, pseudogene \\
\hline MEI4 & 3.213357 & 0.00135 & 0.031558 & 3.205177 & Up & meiotic double-stranded break formation protein 4 \\
\hline PCDHB7 & 3.206851 & 0.001516 & 0.033695 & 3.171611 & Up & protocadherin beta 7 \\
\hline DMBT1L1 & 3.201517 & 0.0005 & 0.018378 & 3.480759 & Up & deleted in malignant brain tumors 1 like 1 (pseudogene) \\
\hline MINAR2 & 3.189907 & 0.001776 & 0.036962 & 3.125332 & Up & membrane integral NOTCH2 associated receptor 2 \\
\hline ESRP1 & 3.186004 & 4.94E-06 & 0.001186 & 4.567488 & Up & epithelial splicing regulatory protein 1 \\
\hline KRT85 & 3.18558 & 0.000405 & 0.016149 & 3.536738 & Up & keratin 85 \\
\hline
\end{tabular}




\begin{tabular}{|c|c|c|c|c|c|c|}
\hline LINC01250 & 3.166207 & 0.001232 & 0.030149 & 3.231272 & Up & long intergenic non-protein coding RNA 1250 \\
\hline ADAM7 & 3.146252 & 0.002852 & 0.047116 & 2.983252 & Up & ADAM metallopeptidase domain 7 \\
\hline EPHA3 & 3.130622 & $2.55 \mathrm{E}-05$ & 0.003077 & 4.210507 & Up & $\mathrm{EPH}$ receptor $\mathrm{A} 3$ \\
\hline SLC7A9 & 3.127796 & 0.000362 & 0.015 & 3.566187 & Up & solute carrier family 7 member 9 \\
\hline PTN & 3.121738 & $6.88 \mathrm{E}-06$ & 0.001442 & 4.497309 & Up & pleiotrophin \\
\hline CEACAM7 & 3.117185 & $8.15 \mathrm{E}-05$ & 0.005999 & 3.940007 & Up & CEA cell adhesion molecule 7 \\
\hline VPS33B-DT & 3.11502 & 0.002578 & 0.044697 & 3.014001 & Up & VPS33B divergent transcript \\
\hline PLEKHN1 & 3.112252 & $2.20 \mathrm{E}-06$ & 0.000683 & 4.733941 & Up & pleckstrin homology domain containing $\mathrm{N} 1$ \\
\hline LOC112268408 & 3.110789 & 0.002661 & 0.045337 & 3.004395 & Up & uncharacterized LOC1 12268408 \\
\hline CNGA1 & 3.108671 & 0.00031 & 0.013808 & 3.606984 & Up & cyclic nucleotide gated channel subunit alpha 1 \\
\hline LOC100507144 & 3.104564 & 0.001868 & 0.038043 & 3.110403 & Up & uncharacterized LOC100507144 \\
\hline RN7SL583P & 3.091743 & 0.0012 & 0.029799 & 3.238954 & Up & RNA, 7SL, cytoplasmic 583, pseudogene \\
\hline CHRNB3 & 3.089636 & 0.002172 & 0.041151 & 3.065643 & Up & cholinergic receptor nicotinic beta 3 subunit \\
\hline FAM133GP & 3.083474 & 0.000613 & 0.020879 & 3.425718 & Up & family with sequence similarity 133 member A pseudogene \\
\hline PWWP3B & 3.082875 & 0.000248 & 0.012032 & 3.66385 & Up & PWWP domain containing 3B \\
\hline GHRHR & 3.074944 & 0.000163 & 0.009601 & 3.770518 & Up & growth hormone releasing hormone receptor \\
\hline LRRC15 & 3.067099 & 0.000274 & 0.012942 & 3.638447 & Up & leucine rich repeat containing 15 \\
\hline SHCBP1L & 3.06246 & 0.000386 & 0.015653 & 3.549279 & Up & SHC binding and spindle associated 1 like \\
\hline MYH4 & 3.059639 & 0.000297 & 0.013428 & 3.618145 & Up & myosin heavy chain 4 \\
\hline CTAGE16P & 3.052947 & 0.001273 & 0.030627 & 3.221993 & Up & CTAGE family member 16 , pseudogene \\
\hline LOC105369203 & 3.047191 & 4.35E-05 & 0.00417 & 4.087835 & Up & uncharacterized LOC105369203 \\
\hline OLA1P2 & 3.046707 & 0.003035 & 0.048798 & 2.964123 & Up & OLA1 pseudogene 2 \\
\hline LOC100129484 & 3.043078 & 0.000217 & 0.011328 & 3.698704 & Up & uncharacterized LOC100129484 \\
\hline MTND6P12 & 3.042631 & 0.001213 & 0.029833 & 3.235749 & Up & MT-ND6 pseudogene 12 \\
\hline CYP4Z2P & 3.034917 & 0.001347 & 0.031558 & 3.205761 & Up & cytochrome $\mathrm{P} 450$ family 4 subfamily $\mathrm{Z}$ member 2 , pseudogene \\
\hline MEIOC & 3.030053 & 0.001193 & 0.029701 & 3.240469 & Up & meiosis specific with coiled-coil domain \\
\hline LOC100505588 & 3.029497 & 0.002764 & 0.046281 & 2.992826 & Up & uncharacterized LOC100505588 \\
\hline MAMDC2 & 3.024368 & 0.000516 & 0.018631 & 3.472303 & Up & MAM domain containing 2 \\
\hline ANKRD66 & 3.02431 & 0.001198 & 0.029776 & 3.23946 & Up & ankyrin repeat domain 66 \\
\hline GPR37L1 & 3.023285 & 3.47E-05 & 0.003684 & 4.140122 & Up & G protein-coupled receptor 37 like 1 \\
\hline CYP26A1 & 3.013734 & 0.001551 & 0.034101 & 3.164983 & Up & cytochrome P450 family 26 subfamily A member 1 \\
\hline SCGB2B2 & 3.008861 & $8.28 \mathrm{E}-05$ & 0.006042 & 3.93614 & Up & secretoglobin family $2 \mathrm{~B}$ member 2 \\
\hline DCAF8L1 & 3.008786 & 0.001977 & 0.039219 & 3.093623 & Up & DDB1 and CUL4 associated factor 8 like 1 \\
\hline CYP2J2 & 3.00746 & 0.002792 & 0.04641 & 2.989729 & Up & cytochrome $\mathrm{P} 450$ family 2 subfamily $\mathrm{J}$ member 2 \\
\hline WDR72 & 2.985403 & $9.18 \mathrm{E}-05$ & 0.006385 & 3.911253 & Up & WD repeat domain 72 \\
\hline CPEB1 & 2.981477 & $5.53 \mathrm{E}-05$ & 0.004728 & 4.031834 & Up & cytoplasmic polyadenylation element binding protein 1 \\
\hline $\mathrm{BNC1}$ & 2.981248 & 0.001138 & 0.028918 & 3.253915 & Up & basonuclin 1 \\
\hline $\mathrm{CCDC} 110$ & 2.980104 & 0.000199 & 0.010794 & 3.720262 & Up & coiled-coil domain containing 110 \\
\hline GLYCAM1 & 2.969315 & 0.002187 & 0.041176 & 3.063582 & Up & glycosylation dependent cell adhesion molecule 1 (pseudogene) \\
\hline CCL22 & 2.961513 & 0.001749 & 0.036613 & 3.12984 & Up & C-C motif chemokine ligand 22 \\
\hline AQP4 & 2.960121 & 4.83E-05 & 0.004447 & 4.063507 & Up & aquaporin 4 \\
\hline FPGT-TNNI3K & 2.96006 & 0.001576 & 0.034401 & 3.160295 & Up & FPGT-TNNI3K readthrough \\
\hline LOC101927884 & 2.947566 & 0.000946 & 0.026225 & 3.306124 & Up & uncharacterized LOC101927884 \\
\hline
\end{tabular}




\begin{tabular}{|c|c|c|c|c|c|c|}
\hline BMPR1B & 2.945795 & $6.42 \mathrm{E}-06$ & 0.001381 & 4.511961 & Up & bone morphogenetic protein receptor type $1 \mathrm{~B}$ \\
\hline SYNPR & 2.938076 & 0.000228 & 0.011442 & 3.68553 & Up & synaptoporin \\
\hline IMPG1 & 2.936346 & 0.000848 & 0.024648 & 3.336789 & Up & interphotoreceptor matrix proteoglycan 1 \\
\hline ANKRD30A & 2.934217 & 0.000437 & 0.016891 & 3.516366 & Up & ankyrin repeat domain $30 \mathrm{~A}$ \\
\hline MYH8 & 2.931689 & 0.000685 & 0.022264 & 3.395619 & Up & myosin heavy chain 8 \\
\hline TMEM105 & 2.924668 & 7.39E-05 & 0.005594 & 3.963279 & Up & TMEM105 long non-coding RNA \\
\hline KRT14 & 2.918362 & 2.29E-06 & 0.000692 & 4.725558 & Up & keratin 14 \\
\hline CLDN2 & 2.91664 & 0.001516 & 0.033695 & 3.17164 & Up & claudin 2 \\
\hline LHFPL3-AS1 & 2.916058 & 0.000512 & 0.018616 & 3.47434 & Up & LHFPL3 antisense RNA 1 \\
\hline SLITRK6 & 2.902106 & 0.003056 & 0.048926 & 2.96206 & Up & SLIT and NTRK like family member 6 \\
\hline DISC1FP1 & 2.894594 & 0.000114 & 0.007337 & 3.858004 & Up & DISC1 fusion partner 1 \\
\hline VGLL2 & 2.894124 & 0.00256 & 0.044663 & 3.016207 & Up & vestigial like family member 2 \\
\hline RBM46 & 2.891768 & 0.001812 & 0.037437 & 3.119353 & Up & RNA binding motif protein 46 \\
\hline MTND5P12 & 2.890344 & $8.48 \mathrm{E}-05$ & 0.006062 & 3.930429 & Up & MT-ND5 pseudogene 12 \\
\hline TCEAL6 & 2.889542 & 0.001046 & 0.027588 & 3.277866 & Up & transcription elongation factor A like 6 \\
\hline ROS1 & 2.886309 & 0.000318 & 0.013947 & 3.600172 & Up & ROS proto-oncogene 1 , receptor tyrosine kinase \\
\hline C10orf53 & 2.884227 & 0.000504 & 0.018497 & 3.478593 & Up & chromosome 10 open reading frame 53 \\
\hline TECRL & 2.882738 & 0.001604 & 0.034896 & 3.15515 & Up & trans-2,3-enoyl-CoA reductase like \\
\hline EYA4 & 2.879404 & 5.97E-05 & 0.00493 & 4.013875 & Up & EYA transcriptional coactivator and phosphatase 4 \\
\hline LOC730100 & 2.875785 & 0.00114 & 0.028943 & 3.253377 & Up & uncharacterized LOC730100 \\
\hline C16orf89 & 2.874808 & 0.002768 & 0.04629 & 2.992389 & Up & chromosome 16 open reading frame 89 \\
\hline KCNH6 & 2.872739 & 0.001298 & 0.03092 & 3.21647 & Up & potassium voltage-gated channel subfamily $\mathrm{H}$ member 6 \\
\hline SCN7A & 2.867538 & 7.78E-05 & 0.005762 & 3.951077 & Up & sodium voltage-gated channel alpha subunit 7 \\
\hline ISM2 & 2.864433 & 0.002963 & 0.048105 & 2.971562 & Up & isthmin 2 \\
\hline GABRG1 & 2.86395 & 0.002861 & 0.047155 & 2.98233 & Up & gamma-aminobutyric acid type A receptor subunit gammal \\
\hline SFTPC & 2.863718 & $3.70 \mathrm{E}-05$ & 0.00378 & 4.125429 & Up & surfactant protein $\mathrm{C}$ \\
\hline CRNDE & 2.863195 & 0.000185 & 0.010247 & 3.738435 & Up & colorectal neoplasia differentially expressed \\
\hline LOC100129175 & 2.851956 & 0.002807 & 0.046533 & 2.988095 & Up & uncharacterized LOC100129175 \\
\hline RIMS2 & 2.850549 & $2.85 \mathrm{E}-05$ & 0.003258 & 4.185403 & Up & regulating synaptic membrane exocytosis 2 \\
\hline CRTC1P1 & 2.850441 & 0.001454 & 0.033004 & 3.183795 & Up & CRTC1 pseudogene 1 \\
\hline SCTR-AS1 & 2.846397 & 0.002011 & 0.039698 & 3.088583 & Up & SCTR antisense RNA 1 \\
\hline LOC339685 & 2.84522 & 0.000513 & 0.018616 & 3.473718 & Up & uncharacterized LOC339685 \\
\hline SCEL & 2.838558 & 0.000784 & 0.023824 & 3.358301 & Up & sciellin \\
\hline TCEAL4P1 & 2.838441 & 0.000176 & 0.010092 & 3.750922 & Up & transcription elongation factor A like 4 pseudogene 1 \\
\hline MYO3A & 2.838051 & $3.82 \mathrm{E}-05$ & 0.00383 & 4.117973 & Up & myosin IIIA \\
\hline ADAM30 & 2.831585 & 0.000892 & 0.025475 & 3.322576 & Up & ADAM metallopeptidase domain 30 \\
\hline $\mathrm{CCDC} 168$ & 2.825794 & 0.000316 & 0.013923 & 3.602088 & Up & coiled-coil domain containing 168 \\
\hline MTRNR2L1 & 2.813509 & 7.11E-08 & 7.93E-05 & 5.388253 & Up & MT-RNR2 like 1 \\
\hline GPR151 & 2.813138 & 0.002917 & 0.047645 & 2.976326 & Up & G protein-coupled receptor 151 \\
\hline NLGN4X & 2.809515 & $2.94 \mathrm{E}-05$ & 0.003321 & 4.178078 & Up & neuroligin $4 \mathrm{X}$-linked \\
\hline ASB5 & 2.80702 & 0.000737 & 0.023226 & 3.375553 & Up & ankyrin repeat and SOCS box containing 5 \\
\hline LBP & 2.802633 & 0.000806 & 0.024108 & 3.350603 & Up & lipopolysaccharide binding protein \\
\hline DELEC1 & 2.801445 & 0.00057 & 0.019974 & 3.445735 & Up & deleted in esophageal cancer 1 \\
\hline
\end{tabular}




\begin{tabular}{|c|c|c|c|c|c|c|}
\hline DMRTA1 & 2.797287 & 0.001398 & 0.032282 & 3.195043 & Up & DMRT like family A1 \\
\hline FAM230B & 2.790151 & 0.000788 & 0.023846 & 3.357094 & Up & family with sequence similarity 230 member B \\
\hline MYF6 & 2.786903 & 0.001221 & 0.02999 & 3.233968 & Up & $\begin{array}{l}\text { myogenic factor } 6 \\
\text { recombination signal binding protein for immunoglobulin kappa } \mathrm{J} \text { region }\end{array}$ \\
\hline RBPJL & 2.781603 & 0.00021 & 0.011148 & 3.706579 & Up & like \\
\hline RP1 & 2.781247 & 0.002073 & 0.040204 & 3.07959 & Up & RP1 axonemal microtubule associated \\
\hline LINC01630 & 2.776756 & 0.001854 & 0.037842 & 3.112712 & Up & long intergenic non-protein coding RNA 1630 \\
\hline CPA4 & 2.775558 & 0.000114 & 0.007333 & 3.858755 & Up & carboxypeptidase A4 \\
\hline TMEM139 & 2.76087 & 0.001088 & 0.02805 & 3.266713 & Up & transmembrane protein 139 \\
\hline SLC9A2 & 2.752986 & 0.002063 & 0.040159 & 3.081041 & Up & solute carrier family 9 member A2 \\
\hline TEKT3 & 2.751933 & 0.000292 & 0.013372 & 3.622295 & Up & tektin 3 \\
\hline FEZF1 & 2.749279 & 0.002606 & 0.044931 & 3.010738 & Up & FEZ family zinc finger 1 \\
\hline SGCD & 2.744477 & $2.77 \mathrm{E}-06$ & 0.000789 & 4.687422 & Up & sarcoglycan delta \\
\hline LOC286177 & 2.743338 & 0.003151 & 0.049748 & 2.952574 & Up & uncharacterized LOC286177 \\
\hline ST8SIA3 & 2.739592 & 4.47E-05 & 0.004229 & 4.081858 & Up & ST8 alpha-N-acetyl-neuraminide alpha-2,8-sialyltransferase 3 \\
\hline SLC5A12 & 2.739076 & 0.001016 & 0.027068 & 3.286118 & Up & solute carrier family 5 member 12 \\
\hline BRINP1 & 2.737461 & 0.000178 & 0.01012 & 3.747803 & Up & BMP/retinoic acid inducible neural specific 1 \\
\hline VXN & 2.736153 & 0.000515 & 0.018631 & 3.473072 & Up & vexin \\
\hline TMEM31 & 2.73473 & 0.002168 & 0.041107 & 3.066193 & Up & transmembrane protein 31 \\
\hline XG & 2.721822 & 0.002188 & 0.041176 & 3.063497 & Up & $\mathrm{Xg}$ glycoprotein (Xg blood group) \\
\hline GPR158 & 2.718024 & 0.000271 & 0.012846 & 3.641144 & Up & G protein-coupled receptor 158 \\
\hline ELAVL2 & 2.710713 & $5.96 \mathrm{E}-05$ & 0.00493 & 4.014458 & Up & ELAV like RNA binding protein 2 \\
\hline ARSJ & 2.710116 & 0.000442 & 0.017056 & 3.513368 & Up & arylsulfatase family member $\mathbf{J}$ \\
\hline ZNF804B & 2.70973 & 0.000289 & 0.013296 & 3.625262 & Up & zinc finger protein $804 \mathrm{~B}$ \\
\hline CFAP300 & 2.708049 & 0.001491 & 0.033412 & 3.176431 & Up & cilia and flagella associated protein 300 \\
\hline GRHL2 & 2.706357 & $5.62 \mathrm{E}-05$ & 0.004783 & 4.028283 & Up & grainyhead like transcription factor 2 \\
\hline LOC400036 & 2.704866 & 0.003135 & 0.049553 & 2.954182 & Up & DEAD-box helicase 23 pseudogene \\
\hline SLC26A5 & 2.703945 & 0.002496 & 0.043986 & 3.023788 & Up & solute carrier family 26 member 5 \\
\hline $\mathrm{EVC}$ & 2.703266 & $6.49 \mathrm{E}-06$ & 0.001384 & 4.509672 & Up & EvC ciliary complex subunit 1 \\
\hline LINC00472 & 2.697899 & 0.000463 & 0.017514 & 3.501345 & Up & long intergenic non-protein coding RNA 472 \\
\hline POU3F3 & 2.697838 & 0.000358 & 0.014969 & 3.569401 & Up & POU class 3 homeobox 3 \\
\hline WNT2 & 2.6951 & 0.000747 & 0.023336 & 3.371581 & Up & Wnt family member 2 \\
\hline LVRN & 2.694329 & 0.000162 & 0.009595 & 3.771991 & Up & $\begin{array}{l}\text { laeverin } \\
\text { killer cell immunoglobulin like recentor three Ig domains and long }\end{array}$ \\
\hline KIR3DL1 & 2.69343 & 0.00156 & 0.034148 & 3.163278 & Up & cytoplasmic tail 1 \\
\hline CBLN1 & 2.691716 & 0.001876 & 0.038108 & 3.109161 & Up & cerebellin 1 precursor \\
\hline SLC36A3 & 2.691472 & 0.001037 & 0.027438 & 3.280157 & Up & solute carrier family 36 member 3 \\
\hline LOC349160 & 2.680699 & 0.001812 & 0.037437 & 3.119408 & Up & uncharacterized LOC349160 \\
\hline DECR2 & 2.680621 & $1.44 \mathrm{E}-06$ & 0.000513 & 4.818799 & Up & 2,4-dienoyl-CoA reductase 2 \\
\hline SLC5A8 & 2.675127 & 0.001786 & 0.037143 & 3.123644 & Up & solute carrier family 5 member 8 \\
\hline CLDN12 & 2.669284 & $7.39 \mathrm{E}-05$ & 0.005594 & 3.963259 & Up & claudin 12 \\
\hline STMND1 & 2.663163 & 0.000418 & 0.016457 & 3.528753 & Up & stathmin domain containing 1 \\
\hline KCTD16 & 2.658392 & 0.000276 & 0.012969 & 3.636715 & Up & potassium channel tetramerization domain containing 16 \\
\hline LRFN5 & 2.657917 & 0.000897 & 0.025499 & 3.321125 & Up & leucine rich repeat and fibronectin type III domain containing 5 \\
\hline
\end{tabular}




\begin{tabular}{|c|c|c|c|c|c|c|}
\hline GLP1R & 2.644488 & 0.000458 & 0.017448 & 3.504402 & Up & glucagon like peptide 1 receptor \\
\hline SCN11A & 2.644333 & 0.000149 & 0.009026 & 3.792542 & Up & sodium voltage-gated channel alpha subunit 11 \\
\hline C3orf56 & 2.644144 & 0.002196 & 0.041176 & 3.062382 & Up & chromosome 3 open reading frame 56 \\
\hline GABRP & 2.643919 & 0.000489 & 0.018121 & 3.486538 & Up & gamma-aminobutyric acid type A receptor subunit pi \\
\hline SLC16A12 & 2.642939 & 0.001471 & 0.033231 & 3.180407 & Up & solute carrier family 16 member 12 \\
\hline AADAT & 2.637926 & 0.000145 & 0.008863 & 3.798904 & Up & aminoadipate aminotransferase \\
\hline LOC101927666 & 2.634652 & 0.000814 & 0.024176 & 3.348034 & Up & uncharacterized LOC101927666 \\
\hline RDH16 & 2.626915 & 0.002563 & 0.044683 & 3.015854 & Up & retinol dehydrogenase 16 \\
\hline DRP2 & 2.621728 & 0.000515 & 0.018631 & 3.472582 & Up & dystrophin related protein 2 \\
\hline PAPOLB & 2.617781 & 0.002102 & 0.040484 & 3.075407 & Up & poly $(\mathrm{A})$ polymerase beta \\
\hline GCM2 & 2.613247 & 0.003104 & 0.049344 & 2.95727 & Up & glial cells missing transcription factor 2 \\
\hline $\mathrm{MC} 1 \mathrm{R}$ & 2.6116 & $4.51 \mathrm{E}-05$ & 0.004256 & 4.079484 & Up & melanocortin 1 receptor \\
\hline DEPDC1-AS1 & 2.606883 & 0.00166 & 0.035644 & 3.145241 & Up & DEPDC1 antisense RNA 1 \\
\hline SERPINA6 & 2.599409 & 0.0029 & 0.04749 & 2.978163 & Up & serpin family A member 6 \\
\hline SLC1A1 & 2.59426 & 0.000757 & 0.023471 & 3.367951 & Up & solute carrier family 1 member 1 \\
\hline CFAP299 & 2.593946 & 0.000632 & 0.021347 & 3.417428 & Up & cilia and flagella associated protein 299 \\
\hline CXCL14 & 2.592708 & 0.001715 & 0.036256 & 3.135663 & Up & C-X-C motif chemokine ligand 14 \\
\hline DSCAM-AS1 & 2.59025 & 0.00272 & 0.04583 & 2.997734 & Up & DSCAM antisense RNA 1 \\
\hline FLG & 2.589226 & 0.000461 & 0.017474 & 3.502371 & Up & filaggrin \\
\hline RGN & 2.584689 & 0.003102 & 0.049344 & 2.957427 & Up & regucalcin \\
\hline RPL6P20 & 2.584134 & 0.002676 & 0.045415 & 3.002708 & Up & ribosomal protein L6 pseudogene 20 \\
\hline LOC102724776 & 2.583449 & 0.003044 & 0.048837 & 2.963305 & Up & uncharacterized LOC102724776 \\
\hline TRIM54 & 2.582009 & 0.001312 & 0.031156 & 3.21345 & Up & tripartite motif containing 54 \\
\hline LOC101928228 & 2.579563 & 0.000177 & 0.010106 & 3.749409 & Up & uncharacterized LOC101928228 \\
\hline RPL26P36 & 2.577256 & 0.001725 & 0.036334 & 3.133878 & Up & ribosomal protein L26 pseudogene 36 \\
\hline SMC1B & 2.577237 & 0.000326 & 0.014131 & 3.594015 & Up & structural maintenance of chromosomes $1 \mathrm{~B}$ \\
\hline PPP1R14C & 2.577061 & 0.000737 & 0.023226 & 3.375252 & Up & protein phosphatase 1 regulatory inhibitor subunit $14 \mathrm{C}$ \\
\hline SHC4 & 2.572754 & 0.001721 & 0.036306 & 3.134546 & Up & SHC adaptor protein 4 \\
\hline CCDC178 & 2.568823 & $5.42 \mathrm{E}-05$ & 0.004666 & 4.036535 & Up & coiled-coil domain containing 178 \\
\hline MUC15 & 2.562581 & 0.000789 & 0.023846 & 3.356556 & Up & mucin 15 , cell surface associated \\
\hline ID4 & 2.562465 & 0.001592 & 0.034669 & 3.157309 & Up & inhibitor of DNA binding 4, HLH protein \\
\hline NPY2R & 2.553344 & 0.000931 & 0.026107 & 3.310675 & Up & neuropeptide $\mathrm{Y}$ receptor $\mathrm{Y} 2$ \\
\hline HPSE2 & 2.550548 & 0.002599 & 0.044877 & 3.011531 & Up & heparanase 2 (inactive) \\
\hline SERPINB13 & 2.547395 & 0.001059 & 0.02763 & 3.27426 & Up & serpin family B member 13 \\
\hline USP43 & 2.543343 & $7.56 \mathrm{E}-05$ & 0.005671 & 3.957821 & Up & ubiquitin specific peptidase 43 \\
\hline TRPM1 & 2.541316 & 0.000644 & 0.021466 & 3.41219 & Up & transient receptor potential cation channel subfamily $\mathrm{M}$ member 1 \\
\hline NEUROG1 & 2.540745 & 0.000433 & 0.016841 & 3.519271 & Up & neurogenin 1 \\
\hline F9 & 2.539726 & 0.0022 & 0.041176 & 3.061785 & Up & coagulation factor IX \\
\hline CREB3L3 & 2.534091 & $9.26 \mathrm{E}-05$ & 0.006392 & 3.909331 & Up & cAMP responsive element binding protein 3 like 3 \\
\hline LSP1P5 & 2.522544 & 0.001436 & 0.032769 & 3.187406 & Up & LSP1 pseudogene 5 \\
\hline $\mathrm{ROBO} 2$ & 2.51639 & $1.66 \mathrm{E}-05$ & 0.002335 & 4.306683 & Up & roundabout guidance receptor 2 \\
\hline GPC6 & 2.515006 & 4.94E-05 & 0.004447 & 4.058525 & Up & glypican 6 \\
\hline RNY3P1 & 2.512029 & $1.54 \mathrm{E}-05$ & 0.002229 & 4.323431 & Up & RNY3 pseudogene 1 \\
\hline
\end{tabular}




\begin{tabular}{|c|c|c|c|c|c|c|}
\hline RPP38-DT & 2.503674 & 0.002047 & 0.040019 & 3.083326 & Up & RPP38 divergent transcript \\
\hline GFPT2 & 2.501221 & 0.000638 & 0.02144 & 3.414845 & Up & glutamine-fructose-6-phosphate transaminase 2 \\
\hline WNT9A & 2.500183 & 0.001349 & 0.031558 & 3.205328 & Up & Wnt family member $9 \mathrm{~A}$ \\
\hline SSX5 & 2.500099 & 0.002573 & 0.044697 & 3.014608 & Up & SSX family member 5 \\
\hline GREM2 & 2.498023 & 0.000434 & 0.016844 & 3.518786 & Up & gremlin 2, DAN family BMP antagonist \\
\hline $\mathrm{KCND} 2$ & 2.497277 & 0.001915 & 0.038394 & 3.103168 & Up & potassium voltage-gated channel subfamily D member 2 \\
\hline PCSK1 & 2.496232 & $6.15 \mathrm{E}-05$ & 0.005023 & 4.007076 & Up & proprotein convertase subtilisin/kexin type 1 \\
\hline PHLDA2 & 2.495562 & 0.001617 & 0.035075 & 3.152878 & Up & pleckstrin homology like domain family A member 2 \\
\hline DDX10P2 & 2.483572 & 0.002193 & 0.041176 & 3.062754 & Up & DEAD-box helicase 10 pseudogene 2 \\
\hline $\mathrm{DACH} 2$ & 2.482729 & 0.000329 & 0.0142 & 3.59169 & Up & dachshund family transcription factor 2 \\
\hline PLA2G2D & 2.47526 & 0.001893 & 0.038276 & 3.106553 & Up & phospholipase A2 group IID \\
\hline CFAP20DC & 2.470592 & 0.000285 & 0.01323 & 3.628628 & Up & CFAP20 domain containing \\
\hline MFAP5 & 2.469399 & $5.16 \mathrm{E}-05$ & 0.004523 & 4.048033 & Up & microfibril associated protein 5 \\
\hline RGPD4-AS1 & 2.465122 & 0.000244 & 0.011968 & 3.668434 & Up & RGPD4 antisense RNA 1 (head to head) \\
\hline GABRB1 & 2.462301 & 0.002572 & 0.044697 & 3.014703 & Up & gamma-aminobutyric acid type A receptor subunit beta 1 \\
\hline AZGP1 & 2.458429 & 0.000824 & 0.024177 & 3.344458 & Up & alpha-2-glycoprotein 1, zinc-binding \\
\hline SLC4A10 & 2.451773 & 2.71E-06 & 0.000789 & 4.691319 & Up & solute carrier family 4 member 10 \\
\hline PTPN3 & 2.450598 & $8.20 \mathrm{E}-06$ & 0.001552 & 4.459976 & Up & protein tyrosine phosphatase non-receptor type 3 \\
\hline C11orf16 & 2.450469 & 0.002817 & 0.04667 & 2.986985 & Up & chromosome 11 open reading frame 16 \\
\hline HAS2 & 2.440967 & 0.002848 & 0.047074 & 2.983733 & Up & hyaluronan synthase 2 \\
\hline CTNNA2 & 2.433397 & 0.001317 & 0.031237 & 3.212327 & Up & catenin alpha 2 \\
\hline IGSF3 & 2.427352 & 0.000182 & 0.010146 & 3.742314 & Up & immunoglobulin superfamily member 3 \\
\hline FNDC7 & 2.42648 & 0.001493 & 0.033435 & 3.17597 & Up & fibronectin type III domain containing 7 \\
\hline IGSF1 & 2.425892 & 0.000118 & 0.007485 & 3.850557 & Up & immunoglobulin superfamily member 1 \\
\hline IGSF21 & 2.422478 & 0.000355 & 0.014893 & 3.571627 & Up & immunoglobin superfamily member 21 \\
\hline SLC5A4 & 2.418933 & 0.001487 & 0.033379 & 3.177249 & Up & solute carrier family 5 member 4 \\
\hline PCDHGA9 & 2.418474 & 0.000377 & 0.015361 & 3.555989 & Up & protocadherin gamma subfamily A, 9 \\
\hline KRT24 & 2.417881 & 0.000634 & 0.02135 & 3.416641 & Up & keratin 24 \\
\hline ANHX & 2.417712 & 0.002621 & 0.045039 & 3.009057 & Up & anomalous homeobox \\
\hline KIT & 2.417683 & 0.000457 & 0.017448 & 3.504662 & Up & KIT proto-oncogene, receptor tyrosine kinase \\
\hline SNX31 & 2.417526 & 0.001533 & 0.03383 & 3.168347 & Up & sorting nexin 31 \\
\hline SLC35F1 & 2.415882 & 0.00229 & 0.042181 & 3.049788 & Up & solute carrier family 35 member F1 \\
\hline $\mathrm{IHO} 1$ & 2.414311 & 0.000753 & 0.023366 & 3.369583 & Up & interactor of HORMAD1 1 \\
\hline ZNF750 & 2.414149 & 0.00103 & 0.027301 & 3.282261 & Up & zinc finger protein 750 \\
\hline FAM71D & 2.413063 & 0.002052 & 0.040029 & 3.082613 & Up & family with sequence similarity 71 member D \\
\hline LONRF2 & 2.409924 & 8.08E-06 & 0.001552 & 4.463113 & Up & LON peptidase $\mathrm{N}$-terminal domain and ring finger 2 \\
\hline TNFAIP8L3 & 2.408773 & $1.42 \mathrm{E}-05$ & 0.002103 & 4.340094 & Up & TNF alpha induced protein 8 like 3 \\
\hline CNTN5 & 2.406147 & 0.000611 & 0.020846 & 3.426521 & Up & contactin 5 \\
\hline PHKA1 & 2.403578 & 5.30E-05 & 0.00461 & 4.04181 & Up & phosphorylase kinase regulatory subunit alpha 1 \\
\hline NCCRP1 & 2.402669 & 0.002145 & 0.0409 & 3.069429 & Up & NCCRP1, F-box associated domain containing \\
\hline RSPO1 & 2.398304 & 2.79E-05 & 0.003258 & 4.189886 & Up & R-spondin 1 \\
\hline GRIA4 & 2.398157 & 0.000935 & 0.02616 & 3.309471 & Up & glutamate ionotropic receptor AMPA type subunit 4 \\
\hline B3GAT1-DT & 2.397891 & 0.001055 & 0.027608 & 3.275302 & Up & B3GAT1 diverget transcript \\
\hline
\end{tabular}




\begin{tabular}{|c|c|c|c|c|c|c|}
\hline ZMAT4 & 2.396372 & 0.00171 & 0.036212 & 3.136383 & Up & zinc finger matrin-type 4 \\
\hline CNTN4 & 2.387406 & 0.000392 & 0.015831 & 3.545439 & Up & contactin 4 \\
\hline PCDH11X & 2.382392 & 0.00082 & 0.024176 & 3.345944 & Up & protocadherin $11 \mathrm{X}$-linked \\
\hline PLAAT5 & 2.370067 & 0.001321 & 0.03128 & 3.211471 & Up & phospholipase A and acyltransferase 5 \\
\hline ENOX1 & 2.367742 & 0.002547 & 0.044513 & 3.017732 & $\mathrm{Up}$ & ecto-NOX disulfide-thiol exchanger 1 \\
\hline LINC02345 & 2.367066 & 0.000762 & 0.023479 & 3.366221 & Up & long intergenic non-protein coding RNA 2345 \\
\hline SLC18A1 & 2.365888 & 0.000427 & 0.016699 & 3.522972 & Up & solute carrier family 18 member A1 \\
\hline SVOP & 2.365594 & 0.002361 & 0.042658 & 3.040602 & Up & $\mathrm{SV} 2$ related protein \\
\hline CFAP53 & 2.365378 & 0.002807 & 0.046533 & 2.988131 & Up & cilia and flagella associated protein 53 \\
\hline CDC20B & 2.364628 & 0.002055 & 0.040046 & 3.082184 & Up & cell division cycle $20 \mathrm{~B}$ \\
\hline OBI1-AS1 & 2.363385 & 0.000182 & 0.010146 & 3.742795 & Up & OBI1 antisense RNA 1 \\
\hline LINC01267 & 2.360519 & 0.002313 & 0.042352 & 3.046768 & Up & long intergenic non-protein coding RNA 1267 \\
\hline NKAPL & 2.359592 & 0.000324 & 0.014101 & 3.595455 & Up & NFKB activating protein like \\
\hline WNT5A & 2.358613 & $7.70 \mathrm{E}-05$ & 0.005717 & 3.95368 & Up & Wnt family member $5 \mathrm{~A}$ \\
\hline ADGRF1 & 2.358543 & 0.002252 & 0.041643 & 3.054814 & Up & adhesion $\mathrm{G}$ protein-coupled receptor $\mathrm{F} 1$ \\
\hline EDA & 2.355859 & 0.000403 & 0.016126 & 3.538198 & Up & ectodysplasin A \\
\hline GNG12-AS1 & 2.355706 & 0.002666 & 0.045355 & 3.003847 & Up & GNG12, DIRAS3 and WLS antisense RNA 1 \\
\hline KCNA1 & 2.350861 & 0.000248 & 0.012032 & 3.664071 & Up & potassium voltage-gated channel subfamily A member 1 \\
\hline CCDC81 & 2.348095 & 0.000479 & 0.017835 & 3.491998 & Up & coiled-coil domain containing 81 \\
\hline VWDE & 2.345836 & 0.00121 & 0.029831 & 3.236534 & Up & von Willebrand factor D and EGF domains \\
\hline TRIM71 & 2.344576 & 0.000719 & 0.022988 & 3.382419 & Up & tripartite motif containing 71 \\
\hline COLEC12 & 2.344077 & 0.000468 & 0.017631 & 3.498331 & Up & collectin subfamily member 12 \\
\hline RASEF & 2.339961 & 0.000762 & 0.023479 & 3.366175 & Up & RAS and EF-hand domain containing \\
\hline MTND6P16 & 2.337388 & 0.002778 & 0.046321 & 2.991281 & Up & MT-ND6 pseudogene 16 \\
\hline LOC101928850 & 2.337377 & 0.001745 & 0.036561 & 3.13054 & Up & uncharacterized LOC101928850 \\
\hline TDRD6 & 2.335919 & 0.000981 & 0.026733 & 3.29581 & Up & tudor domain containing 6 \\
\hline LINC01121 & 2.335434 & 0.000179 & 0.01012 & 3.747306 & Up & long intergenic non-protein coding RNA 1121 \\
\hline SCN1A & 2.332824 & 0.002505 & 0.043986 & 3.022779 & Up & sodium voltage-gated channel alpha subunit 1 \\
\hline MTND2P13 & 2.324998 & 0.000311 & 0.013819 & 3.606314 & Up & MT-ND2 pseudogene 13 \\
\hline LOC100505715 & 2.316277 & 0.001178 & 0.029564 & 3.24411 & Up & uncharacterized LOC100505715 \\
\hline MTRNR2L2 & 2.313195 & $6.44 \mathrm{E}-05$ & 0.005153 & 3.996031 & Up & MT-RNR2 like 2 \\
\hline $\mathrm{MROH} 2 \mathrm{~B}$ & 2.309703 & 0.002021 & 0.039824 & 3.087165 & Up & maestro heat like repeat family member $2 \mathrm{~B}$ \\
\hline ATP13A5 & 2.30919 & 0.002466 & 0.043601 & 3.027536 & Up & ATPase 13A5 \\
\hline MTCYBP35 & 2.309142 & 0.001691 & 0.035914 & 3.13982 & Up & MT-CYB pseudogene 35 \\
\hline ITIH3 & 2.303498 & 0.000401 & 0.016091 & 3.53941 & Up & inter-alpha-trypsin inhibitor heavy chain 3 \\
\hline TBX18 & 2.298837 & 0.001521 & 0.033725 & 3.17056 & Up & T-box transcription factor 18 \\
\hline RGPD8 & 2.298546 & $1.34 \mathrm{E}-05$ & 0.002019 & 4.353131 & Up & RANBP2 like and GRIP domain containing 8 \\
\hline MTRNR2L8 & 2.291085 & $8.26 \mathrm{E}-05$ & 0.006042 & 3.936666 & Up & MT-RNR2 like 8 \\
\hline HSD11B2 & 2.288685 & 0.001905 & 0.038276 & 3.104702 & Up & hydroxysteroid 11-beta dehydrogenase 2 \\
\hline LOC100128164 & 2.285659 & 0.000173 & 0.009978 & 3.754942 & Up & four and a half LIM domains 1 pseudogene \\
\hline VWA5B1 & 2.285369 & 0.000224 & 0.011379 & 3.690841 & Up & von Willebrand factor A domain containing 5B1 \\
\hline FRMPD1 & 2.277255 & 0.001898 & 0.038276 & 3.105754 & Up & FERM and PDZ domain containing 1 \\
\hline NUDT10 & 2.27087 & 0.002654 & 0.045307 & 3.005226 & Up & nudix hydrolase 10 \\
\hline
\end{tabular}




\begin{tabular}{|c|c|c|c|c|c|c|}
\hline $\mathrm{SCN} 2 \mathrm{~A}$ & 2.266149 & 0.001513 & 0.033695 & 3.172115 & Up & sodium voltage-gated channel alpha subunit 2 \\
\hline LRP1B & 2.263645 & 0.000622 & 0.021084 & 3.421929 & Up & LDL receptor related protein $1 \mathrm{~B}$ \\
\hline TUSC 8 & 2.26322 & 0.000594 & 0.020535 & 3.4344 & Up & tumor suppressor candidate 8 \\
\hline TMC7 & 2.259069 & 0.000565 & 0.019862 & 3.447645 & Up & transmembrane channel like 7 \\
\hline DNAH8 & 2.257525 & 2.87E-05 & 0.003258 & 4.18394 & Up & dynein axonemal heavy chain 8 \\
\hline TACC2 & 2.249898 & $9.64 \mathrm{E}-08$ & $9.09 \mathrm{E}-05$ & 5.333445 & Up & transforming acidic coiled-coil containing protein 2 \\
\hline SRRM1P1 & 2.240683 & 0.000165 & 0.009637 & 3.767174 & Up & serine/arginine repetitive matrix 1 pseudogene 1 \\
\hline KCNK1 & 2.237479 & 0.002714 & 0.045771 & 2.998453 & Up & potassium two pore domain channel subfamily $\mathrm{K}$ member 1 \\
\hline RPL31P2 & 2.237463 & 0.002088 & 0.040327 & 3.077352 & Up & ribosomal protein L31 pseudogene 2 \\
\hline SHISAL2B & 2.232438 & 0.000662 & 0.021852 & 3.404741 & Up & shisa like $2 \mathrm{~B}$ \\
\hline ADGRV1 & 2.22211 & 0.000267 & 0.01268 & 3.645087 & Up & adhesion $\mathrm{G}$ protein-coupled receptor $\mathrm{V} 1$ \\
\hline LMCD1-AS1 & 2.220982 & 0.002039 & 0.039991 & 3.084557 & Up & LMCD1 antisense RNA 1 \\
\hline PTHLH & 2.220149 & 0.002684 & 0.045476 & 3.001776 & Up & parathyroid hormone like hormone \\
\hline CCNA1 & 2.218492 & 0.002222 & 0.041364 & 3.058791 & Up & cyclin A1 \\
\hline LOC101929331 & 2.216569 & 0.001331 & 0.031467 & 3.209205 & Up & uncharacterized LOC101929331 \\
\hline FANK1 & 2.209783 & 0.001076 & 0.027879 & 3.269923 & Up & fibronectin type III and ankyrin repeat domains 1 \\
\hline COBL & 2.208342 & $2.38 \mathrm{E}-05$ & 0.003075 & 4.22623 & Up & cordon-bleu WH2 repeat protein \\
\hline CA12 & 2.204549 & 0.001852 & 0.037842 & 3.113031 & Up & carbonic anhydrase 12 \\
\hline ACVR1C & 2.202767 & 0.000159 & 0.009489 & 3.777069 & Up & activin A receptor type $1 \mathrm{C}$ \\
\hline LIN28B & 2.19221 & 0.002343 & 0.042557 & 3.04297 & Up & $\operatorname{lin}-28$ homolog B \\
\hline FAP & 2.190262 & 0.002649 & 0.045307 & 3.005779 & Up & fibroblast activation protein alpha \\
\hline $\mathrm{CDH} 8$ & 2.188607 & 0.000822 & 0.024176 & 3.345133 & Up & cadherin 8 \\
\hline LINC02516 & 2.188282 & 0.001885 & 0.038231 & 3.107719 & Up & long intergenic non-protein coding RNA 2516 \\
\hline HUNK & 2.186613 & 0.001559 & 0.034147 & 3.163546 & Up & hormonally up-regulated Neu-associated kinase \\
\hline GDA & 2.18637 & 0.000475 & 0.017713 & 3.494649 & Up & guanine deaminase \\
\hline FERMT1 & 2.186142 & 0.001741 & 0.036551 & 3.131129 & Up & fermitin family member 1 \\
\hline PCDHAC2 & 2.182501 & 0.00315 & 0.049748 & 2.952745 & Up & protocadherin alpha subfamily $\mathrm{C}, 2$ \\
\hline DACT3 & 2.180069 & 0.000724 & 0.022988 & 3.380384 & Up & dishevelled binding antagonist of beta catenin 3 \\
\hline THSD7A & 2.174731 & $3.78 \mathrm{E}-05$ & 0.003827 & 4.120684 & Up & thrombospondin type 1 domain containing 7A \\
\hline IQCH & 2.17072 & 0.00026 & 0.012401 & 3.652197 & Up & IQ motif containing $\mathrm{H}$ \\
\hline EFCAB5 & 2.166213 & 0.000248 & 0.012032 & 3.664178 & Up & EF-hand calcium binding domain 5 \\
\hline PEX5L & 2.159847 & 0.001799 & 0.037314 & 3.12155 & Up & peroxisomal biogenesis factor 5 like \\
\hline KRT80 & 2.157287 & 0.001077 & 0.027891 & 3.269503 & Up & keratin 80 \\
\hline DGKB & 2.152441 & 0.002866 & 0.047155 & 2.981723 & Up & diacylglycerol kinase beta \\
\hline MIPOL1 & 2.151576 & $3.96 \mathrm{E}-07$ & 0.000202 & 5.070865 & Up & mirror-image polydactyly 1 \\
\hline ERVFRD-1 & 2.146361 & 0.002069 & 0.04018 & 3.080109 & Up & endogenous retrovirus group FRD member 1, envelope \\
\hline MROH7 & 2.144137 & 0.001891 & 0.038276 & 3.106885 & Up & maestro heat like repeat family member 7 \\
\hline NWD1 & 2.138886 & 0.001529 & 0.033794 & 3.169182 & Up & NACHT and WD repeat domain containing 1 \\
\hline RPL5P9 & 2.136476 & 0.001417 & 0.032481 & 3.191203 & $\mathrm{Up}$ & ribosomal protein L5 pseudogene 9 \\
\hline IRX2 & 2.128446 & 0.00267 & 0.04539 & 3.003404 & $\mathrm{Up}$ & iroquois homeobox 2 \\
\hline C8orf34 & 2.126376 & 0.000638 & 0.02144 & 3.414745 & Up & chromosome 8 open reading frame 34 \\
\hline $\mathrm{ABCA} 4$ & 2.11746 & 0.001117 & 0.028579 & 3.259331 & Up & ATP binding cassette subfamily A member 4 \\
\hline STRC & 2.116012 & 0.003071 & 0.049015 & 2.960547 & Up & stereocilin \\
\hline
\end{tabular}




\begin{tabular}{|c|c|c|c|c|c|c|}
\hline PTH2R & 2.115792 & 0.000304 & 0.013588 & 3.612101 & Up & parathyroid hormone 2 receptor \\
\hline NXPE1 & 2.113269 & 0.002283 & 0.042123 & 3.05065 & Up & neurexophilin and PC-esterase domain family member 1 \\
\hline TTC6 & 2.111884 & 0.001845 & 0.03779 & 3.114092 & Up & tetratricopeptide repeat domain 6 \\
\hline TMEM117 & 2.111578 & 0.001239 & 0.030205 & 3.229725 & Up & transmembrane protein 117 \\
\hline HPGDS & 2.109892 & 0.002109 & 0.040552 & 3.074442 & Up & hematopoietic prostaglandin D synthase \\
\hline LINC00319 & 2.107732 & 0.002376 & 0.042799 & 3.038703 & Up & long intergenic non-protein coding RNA 319 \\
\hline POPDC2 & 2.105552 & 0.001277 & 0.030666 & 3.221072 & Up & popeye domain containing 2 \\
\hline ADCY2 & 2.105369 & 0.002746 & 0.046108 & 2.994785 & Up & adenylate cyclase 2 \\
\hline NEUROD1 & 2.103703 & 0.001825 & 0.03757 & 3.117299 & Up & neuronal differentiation 1 \\
\hline DYDC2 & 2.103538 & 0.002326 & 0.042463 & 3.04516 & Up & DPY30 domain containing 2 \\
\hline ANKS1B & 2.103506 & 0.000361 & 0.015 & 3.566711 & Up & ankyrin repeat and sterile alpha motif domain containing $1 \mathrm{~B}$ \\
\hline SPINK5 & 2.091698 & 3.77E-06 & 0.000972 & 4.623788 & Up & serine peptidase inhibitor Kazal type 5 \\
\hline DNAH7 & 2.083609 & 0.000664 & 0.021877 & 3.404061 & Up & dynein axonemal heavy chain 7 \\
\hline KCNIP3 & 2.080304 & 0.000407 & 0.016188 & 3.535437 & Up & potassium voltage-gated channel interacting protein 3 \\
\hline EFHD1 & 2.079688 & 0.000366 & 0.015111 & 3.563362 & Up & EF-hand domain family member D1 \\
\hline PTPRVP & 2.07093 & 0.001905 & 0.038276 & 3.104597 & Up & protein tyrosine phosphatase receptor type $\mathrm{V}$, pseudogene \\
\hline IL20RA & 2.059487 & 0.001232 & 0.030149 & 3.231432 & Up & interleukin 20 receptor subunit alpha \\
\hline CLDN1 & 2.051894 & 0.00046 & 0.017474 & 3.502794 & Up & claudin 1 \\
\hline MAL2 & 2.045176 & 0.00094 & 0.026167 & 3.307794 & Up & mal, $\mathrm{T}$ cell differentiation protein 2 \\
\hline IZUMO1 & 2.04049 & 0.002425 & 0.043302 & 3.032495 & Up & izumo sperm-egg fusion 1 \\
\hline SHROOM3 & 2.036624 & 0.000774 & 0.023633 & 3.361906 & Up & shroom family member 3 \\
\hline KLK11 & 2.03325 & 0.001722 & 0.036306 & 3.134359 & Up & kallikrein related peptidase 11 \\
\hline CCNE1 & 2.031649 & $8.59 \mathrm{E}-05$ & 0.006125 & 3.927182 & Up & cyclin E1 \\
\hline C5orf34 & 2.030955 & 0.001251 & 0.030269 & 3.227007 & Up & chromosome 5 open reading frame 34 \\
\hline HAGLR & 2.030678 & 0.000244 & 0.011968 & 3.668631 & Up & HOXD antisense growth-associated long non-coding RNA \\
\hline SOHLH1 & 2.027269 & 0.002949 & 0.048 & 2.973046 & Up & spermatogenesis and oogenesis specific basic helix-loop-helix 1 \\
\hline SLC6A7 & 2.026896 & 0.002113 & 0.040594 & 3.073894 & Up & solute carrier family 6 member 7 \\
\hline DISP3 & 2.017289 & 0.003171 & 0.049996 & 2.950641 & Up & dispatched RND transporter family member 3 \\
\hline CCDC144A & 2.017023 & 0.002696 & 0.045569 & 3.000434 & Up & coiled-coil domain containing 144A \\
\hline RNY3P15 & 2.012765 & 0.000488 & 0.018098 & 3.487286 & Up & RNY3 pseudogene 15 \\
\hline FUT3 & 2.00891 & 0.001339 & 0.031528 & 3.20754 & Up & fucosyltransferase 3 (Lewis blood group) \\
\hline GABRB3 & 1.999488 & 0.00036 & 0.015 & 3.567729 & Up & gamma-aminobutyric acid type A receptor subunit beta3 \\
\hline ANKRD45 & 1.998307 & 0.002582 & 0.044697 & 3.01351 & Up & ankyrin repeat domain 45 \\
\hline SLC7A14 & 1.997464 & 0.00134 & 0.031529 & 3.207166 & Up & solute carrier family 7 member 14 \\
\hline ETV1 & 1.994147 & 0.000931 & 0.026107 & 3.310687 & Up & ETS variant transcription factor 1 \\
\hline CYP24A1 & 1.99389 & 0.001803 & 0.037364 & 3.120901 & Up & cytochrome P450 family 24 subfamily A member 1 \\
\hline TNR & 1.989575 & 0.000117 & 0.007455 & 3.852826 & Up & tenascin R \\
\hline ERRFI1 & 1.986467 & 0.000163 & 0.009601 & 3.770865 & Up & ERBB receptor feedback inhibitor 1 \\
\hline PKHD1 & 1.981313 & 0.001264 & 0.030438 & 3.224052 & Up & PKHD1 ciliary IPT domain containing fibrocystin/polyductin \\
\hline RNY4P3 & 1.980563 & 0.000528 & 0.018925 & 3.466125 & Up & RNY4 pseudogene 3 \\
\hline KRT13 & 1.980125 & 0.00216 & 0.041044 & 3.067343 & Up & keratin 13 \\
\hline SLIT2 & 1.977635 & 0.000946 & 0.026225 & 3.306227 & Up & slit guidance ligand 2 \\
\hline C2orf72 & 1.973621 & 0.001796 & 0.037284 & 3.122029 & Up & chromosome 2 open reading frame 72 \\
\hline
\end{tabular}




\begin{tabular}{|c|c|c|c|c|c|c|}
\hline MAST1 & 1.967185 & 0.001674 & 0.035744 & 3.142734 & Up & microtubule associated serine/threonine kinase 1 \\
\hline DTNA & 1.965549 & $3.74 \mathrm{E}-06$ & 0.000972 & 4.625455 & Up & dystrobrevin alpha \\
\hline MTND6P3 & 1.954429 & 0.000989 & 0.026772 & 3.293577 & Up & MT-ND6 pseudogene 3 \\
\hline ANTXR1 & 1.953571 & 0.000557 & 0.019657 & 3.451608 & Up & ANTXR cell adhesion molecule 1 \\
\hline ITGA8 & 1.943705 & 0.000215 & 0.011264 & 3.70068 & $\mathrm{Up}$ & integrin subunit alpha 8 \\
\hline KCNA7 & 1.943639 & 0.001181 & 0.029566 & 3.243516 & Up & potassium voltage-gated channel subfamily A member 7 \\
\hline RPL5P5 & 1.938703 & 0.001865 & 0.038009 & 3.110912 & Up & ribosomal protein L5 pseudogene 5 \\
\hline MIR100HG & 1.931116 & 0.000802 & 0.024074 & 3.352008 & Up & mir-100-let-7a-2-mir-125b-1 cluster host gene \\
\hline MTND6P7 & 1.926584 & 0.002089 & 0.040327 & 3.077268 & Up & MT-ND6 pseudogene 7 \\
\hline GJC1 & 1.925965 & 0.002785 & 0.046349 & 2.990545 & Up & gap junction protein gamma 1 \\
\hline WNK3 & 1.911953 & 0.000303 & 0.013583 & 3.612905 & Up & WNK lysine deficient protein kinase 3 \\
\hline PPARGC1A & 1.909247 & 0.001337 & 0.031528 & 3.207817 & Up & PPARG coactivator 1 alpha \\
\hline TRPM3 & 1.894528 & 0.001056 & 0.027608 & 3.275048 & Up & transient receptor potential cation channel subfamily $\mathrm{M}$ member 3 \\
\hline PAK3 & 1.886078 & 0.000506 & 0.018497 & 3.47747 & Up & p21 (RAC1) activated kinase 3 \\
\hline IGF1 & 1.885985 & 4.94E-05 & 0.004447 & 4.058211 & $\mathrm{Up}$ & insulin like growth factor 1 \\
\hline LRRC7 & 1.885084 & 0.002344 & 0.042557 & 3.042833 & Up & leucine rich repeat containing 7 \\
\hline UNC80 & 1.884923 & 0.000824 & 0.024177 & 3.344691 & Up & unc-80 homolog, NALCN channel complex subunit \\
\hline PRKN & 1.883231 & 0.001161 & 0.029354 & 3.248193 & Up & parkin RBR E3 ubiquitin protein ligase \\
\hline CCDC114 & 1.878413 & 0.001388 & 0.032202 & 3.197052 & $\mathrm{Up}$ & coiled-coil domain containing 114 \\
\hline POU2F3 & 1.867221 & 0.002618 & 0.045039 & 3.009403 & Up & POU class 2 homeobox 3 \\
\hline LINC00261 & 1.864563 & 0.002192 & 0.041176 & 3.062916 & Up & long intergenic non-protein coding RNA 261 \\
\hline SIGLEC5 & 1.864333 & 0.002118 & 0.0406 & 3.073196 & Up & sialic acid binding Ig like lectin 5 \\
\hline OSR1 & 1.859268 & 0.00108 & 0.027939 & 3.268722 & Up & odd-skipped related transcription factor 1 \\
\hline RNA5SP202 & 1.857863 & $2.58 \mathrm{E}-05$ & 0.003086 & 4.207956 & Up & RNA, 5S ribosomal pseudogene 202 \\
\hline ZNF599 & 1.852715 & 0.000256 & 0.012271 & 3.65655 & Up & zinc finger protein 599 \\
\hline HRG & 1.845664 & 0.002023 & 0.039829 & 3.086884 & Up & histidine rich glycoprotein \\
\hline B3GNT4 & 1.841526 & 0.002328 & 0.042463 & 3.044899 & Up & UDP-GlcNAc:betaGal beta-1,3-N-acetylglucosaminyltransferase 4 \\
\hline CYP46A1 & 1.823515 & 0.002982 & 0.048357 & 2.969551 & Up & cytochrome P450 family 46 subfamily A member 1 \\
\hline SH3RF2 & 1.819235 & 0.001247 & 0.030249 & 3.227811 & Up & SH3 domain containing ring finger 2 \\
\hline TTC25 & 1.818112 & 0.001302 & 0.030956 & 3.215577 & Up & tetratricopeptide repeat domain 25 \\
\hline CDH6 & 1.817889 & 0.002522 & 0.044225 & 3.020705 & Up & cadherin 6 \\
\hline FHAD1 & 1.807752 & 0.001379 & 0.032073 & 3.199029 & Up & forkhead associated phosphopeptide binding domain 1 \\
\hline FOLR2 & 1.806169 & 0.001876 & 0.038108 & 3.109204 & Up & folate receptor beta \\
\hline INAVA & 1.80001 & 0.000708 & 0.02286 & 3.386569 & Up & innate immunity activator \\
\hline ARHGEF4 & 1.797538 & 0.001557 & 0.034141 & 3.163862 & Up & Rho guanine nucleotide exchange factor 4 \\
\hline CSRP2 & 1.797297 & 0.00078 & 0.023744 & 3.359926 & Up & cysteine and glycine rich protein 2 \\
\hline SETD9 & 1.788362 & 0.000831 & 0.024307 & 3.342201 & Up & SET domain containing 9 \\
\hline TRPV6 & 1.786255 & 0.002295 & 0.042181 & 3.049106 & Up & transient receptor potential cation channel subfamily $\mathrm{V}$ member 6 \\
\hline ATP1A4 & 1.786037 & 0.001294 & 0.030853 & 3.217366 & Up & ATPase $\mathrm{Na}+\mathrm{K}+$ transporting subunit alpha 4 \\
\hline TUFT1 & 1.785546 & 0.00045 & 0.017213 & 3.508951 & Up & tuftelin 1 \\
\hline TLR3 & 1.778403 & 0.000288 & 0.013293 & 3.625928 & Up & toll like receptor 3 \\
\hline CIART & 1.777924 & 0.002992 & 0.048449 & 2.96854 & Up & circadian associated repressor of transcription \\
\hline BNIPL & 1.770915 & 0.001501 & 0.033537 & 3.17456 & Up & BCL2 interacting protein like \\
\hline
\end{tabular}




\begin{tabular}{|c|c|c|c|c|c|c|}
\hline ZBTB8A & 1.766359 & 0.002771 & 0.046306 & 2.992076 & Up & zinc finger and BTB domain containing 8A \\
\hline ANKRD11P1 & 1.757155 & 0.00126 & 0.030383 & 3.224854 & Up & ANKRD11 pseudogene 1 \\
\hline BMP7 & 1.754203 & 0.0025 & 0.043986 & 3.023331 & Up & bone morphogenetic protein 7 \\
\hline GNAL & 1.753 & 0.000812 & 0.024176 & 3.348706 & Up & G protein subunit alpha $\mathrm{L}$ \\
\hline DDX11L16 & 1.748222 & 0.000834 & 0.024307 & 3.341316 & $\mathrm{Up}$ & DEAD/H-box helicase 11 like 16 (pseudogene) \\
\hline DSG2 & 1.745653 & 0.002241 & 0.041536 & 3.056218 & Up & desmoglein 2 \\
\hline CELF5 & 1.742927 & 0.002082 & 0.040319 & 3.078226 & Up & CUGBP Elav-like family member 5 \\
\hline POSTN & 1.741624 & 0.002116 & 0.0406 & 3.07341 & Up & periostin \\
\hline DZANK1 & 1.734506 & 0.002043 & 0.039991 & 3.083933 & Up & double zinc ribbon and ankyrin repeat domains 1 \\
\hline STOML1 & 1.732747 & 0.001133 & 0.028852 & 3.255148 & Up & stomatin like 1 \\
\hline $\mathrm{CCDC} 150$ & 1.731692 & 0.0002 & 0.010815 & 3.719213 & Up & coiled-coil domain containing 150 \\
\hline MTND2P28 & -4.90599 & $6.27 \mathrm{E}-18$ & $1.54 \mathrm{E}-13$ & -8.62753 & Down & MT-ND2 pseudogene 28 \\
\hline SNORD46 & -4.03607 & $1.87 \mathrm{E}-06$ & 0.000616 & -4.76672 & Down & small nucleolar RNA, C/D box 46 \\
\hline SCARNA21 & -3.96374 & $1.46 \mathrm{E}-07$ & 0.000108 & -5.25768 & Down & small Cajal body-specific RNA 21 \\
\hline MTND1P23 & -3.65224 & $6.88 \mathrm{E}-07$ & 0.000307 & -4.96464 & Down & MT-ND1 pseudogene 23 \\
\hline COX4I2 & -3.46711 & 0.00184 & 0.03779 & -3.1149 & Down & cytochrome c oxidase subunit $4 \mathrm{I} 2$ \\
\hline SNORD15B & -3.36678 & $3.40 \mathrm{E}-12$ & $2.40 \mathrm{E}-08$ & -6.96024 & Down & small nucleolar RNA, C/D box 15B \\
\hline SNORD67 & -3.27752 & $8.82 \mathrm{E}-05$ & 0.006199 & -3.92082 & Down & small nucleolar RNA, C/D box 67 \\
\hline SNORA26 & -3.16261 & 0.000292 & 0.013372 & -3.62225 & Down & small nucleolar RNA, H/ACA box 26 \\
\hline SCARNA7 & -3.12296 & $5.75 \mathrm{E}-08$ & $7.28 \mathrm{E}-05$ & -5.42628 & Down & small Cajal body-specific RNA 7 \\
\hline SCARNA6 & -3.1138 & $3.69 \mathrm{E}-06$ & 0.000972 & -4.62793 & Down & small Cajal body-specific RNA 6 \\
\hline SNORA49 & -3.00158 & $1.32 \mathrm{E}-05$ & 0.001992 & -4.35741 & Down & small nucleolar RNA, H/ACA box 49 \\
\hline SNORD89 & -2.98616 & $3.22 \mathrm{E}-07$ & 0.000179 & -5.1101 & Down & small nucleolar RNA, C/D box 89 \\
\hline XIST & -2.94715 & $2.25 \mathrm{E}-08$ & 4.04E-05 & -5.59163 & Down & $\mathrm{X}$ inactive specific transcript \\
\hline MTCO1P12 & -2.91868 & $6.76 \mathrm{E}-10$ & $1.84 \mathrm{E}-06$ & -6.17155 & Down & MT-CO1 pseudogene 12 \\
\hline SNORA46 & -2.83296 & $2.27 \mathrm{E}-05$ & 0.00299 & -4.23664 & Down & small nucleolar RNA, H/ACA box 46 \\
\hline SNORD8 & -2.80183 & 0.000218 & 0.011333 & -3.69697 & Down & small nucleolar RNA, C/D box 8 \\
\hline LTA & -2.74322 & 0.002893 & 0.047414 & -2.97886 & Down & lymphotoxin alpha \\
\hline SNORA20 & -2.73344 & 0.000125 & 0.007836 & -3.83615 & Down & small nucleolar RNA, H/ACA box 20 \\
\hline SNORA75 & -2.70965 & 0.001906 & 0.038276 & -3.10457 & Down & small nucleolar RNA, H/ACA box 75 \\
\hline SNORD116-24 & -2.70339 & 0.002652 & 0.045307 & -3.00541 & Down & small nucleolar RNA, C/D box 116-24 \\
\hline SNORD17 & -2.68133 & $3.92 \mathrm{E}-12$ & $2.40 \mathrm{E}-08$ & -6.94007 & Down & small nucleolar RNA, C/D box 17 \\
\hline SNORA53 & -2.62376 & $1.12 \mathrm{E}-07$ & $9.13 \mathrm{E}-05$ & -5.30657 & Down & small nucleolar RNA, H/ACA box 53 \\
\hline SNORA23 & -2.55895 & $3.17 \mathrm{E}-07$ & 0.000179 & -5.11277 & Down & small nucleolar RNA, H/ACA box 23 \\
\hline SNORA12 & -2.34359 & $1.48 \mathrm{E}-06$ & 0.000518 & -4.81391 & Down & small nucleolar RNA, H/ACA box 12 \\
\hline SNORD9 & -2.31392 & 0.00296 & 0.048102 & -2.97188 & Down & small nucleolar RNA, C/D box 9 \\
\hline SCARNA10 & -2.30272 & $1.68 \mathrm{E}-07$ & 0.000114 & -5.23166 & Down & small Cajal body-specific RNA 10 \\
\hline SNORA73B & -2.27776 & $5.25 \mathrm{E}-06$ & 0.001225 & -4.55464 & Down & small nucleolar RNA, H/ACA box 73B \\
\hline RNU5A-1 & -2.2381 & $2.58 \mathrm{E}-05$ & 0.003086 & -4.20766 & Down & RNA, U5A small nuclear 1 \\
\hline LOC400541 & -2.13572 & 0.002581 & 0.044697 & -3.01371 & Down & uncharacterized LOC400541 \\
\hline $\mathrm{CCDC} 24$ & -2.06616 & 0.00103 & 0.027301 & -3.28218 & Down & coiled-coil domain containing 24 \\
\hline CXCL5 & -2.00406 & $1.17 \mathrm{E}-06$ & 0.000443 & -4.86103 & Down & $\mathrm{C}-\mathrm{X}-\mathrm{C}$ motif chemokine ligand 5 \\
\hline MALAT1 & -1.96132 & $2.47 \mathrm{E}-08$ & 4.04E-05 & -5.5753 & Down & metastasis associated lung adenocarcinoma transcript 1 \\
\hline
\end{tabular}




\begin{tabular}{|c|c|c|c|c|c|c|}
\hline ITGA2B & -1.95681 & $3.00 \mathrm{E}-07$ & 0.000175 & -5.12318 & Down & integrin subunit alpha $2 b$ \\
\hline TMEM70 & -1.9551 & 7.91E-07 & 0.00034 & -4.93767 & Down & transmembrane protein 70 \\
\hline TMEM150A & -1.87702 & 0.000292 & 0.013372 & -3.62198 & Down & transmembrane protein $150 \mathrm{~A}$ \\
\hline LGALS3BP & -1.87374 & 7.10E-05 & 0.005475 & -3.97284 & Down & galectin 3 binding protein \\
\hline ITGB3 & -1.82598 & 4.35E-07 & 0.000209 & -5.05282 & Down & integrin subunit beta 3 \\
\hline CCDC28B & -1.81435 & 0.001027 & 0.027283 & -3.28297 & Down & coiled-coil domain containing $28 \mathrm{~B}$ \\
\hline CNNM4 & -1.8064 & 0.000351 & 0.014776 & -3.57459 & Down & cyclin and CBS domain divalent metal cation transport mediator 4 \\
\hline SPSB2 & -1.80517 & 0.00019 & 0.01042 & -3.73252 & Down & splA/ryanodine receptor domain and SOCS box containing 2 \\
\hline SEC14L5 & -1.77994 & $5.86 \mathrm{E}-05$ & 0.004918 & -4.01829 & Down & SEC14 like lipid binding 5 \\
\hline PLXNB2 & -1.76414 & 5.94E-08 & 7.28E-05 & -5.42064 & Down & plexin B2 \\
\hline MPL & -1.69211 & $1.12 \mathrm{E}-05$ & 0.001787 & -4.39349 & Down & MPL proto-oncogene, thrombopoietin receptor \\
\hline UBOX5 & -1.68768 & 0.000939 & 0.026167 & -3.30826 & Down & U-box domain containing 5 \\
\hline MTCO2P12 & -1.6563 & $2.51 \mathrm{E}-05$ & 0.003077 & -4.21361 & Down & MT-CO2 pseudogene 12 \\
\hline EXO5 & -1.63808 & 0.003087 & 0.049175 & -2.95895 & Down & exonuclease 5 \\
\hline TMEM178A & -1.6373 & 0.002427 & 0.043302 & -3.03227 & Down & transmembrane protein $178 \mathrm{~A}$ \\
\hline NEAT1 & -1.63692 & $6.53 \mathrm{E}-11$ & $3.20 \mathrm{E}-07$ & -6.53097 & Down & nuclear paraspeckle assembly transcript 1 \\
\hline ITGAX & -1.63477 & $3.35 \mathrm{E}-10$ & $1.17 \mathrm{E}-06$ & -6.28152 & Down & integrin subunit alpha $X$ \\
\hline IGHD & -1.55784 & $1.78 \mathrm{E}-06$ & 0.000606 & -4.77683 & Down & immunoglobulin heavy constant delta \\
\hline F13A1 & -1.55093 & $1.03 \mathrm{E}-05$ & 0.001765 & -4.41066 & Down & coagulation factor XIII A chain \\
\hline POMK & -1.54862 & 0.000992 & 0.026772 & -3.29291 & Down & protein O-mannose kinase \\
\hline MPIG6B & -1.54393 & $2.05 \mathrm{E}-05$ & 0.002771 & -4.25979 & Down & megakaryocyte and platelet inhibitory receptor G6b \\
\hline SCARNA5 & -1.53993 & 0.001063 & 0.027659 & -3.27336 & Down & small Cajal body-specific RNA 5 \\
\hline THBS1 & -1.53355 & 2.71E-07 & 0.000162 & -5.14246 & Down & thrombospondin 1 \\
\hline FLNA & -1.5138 & $3.81 \mathrm{E}-10$ & $1.17 \mathrm{E}-06$ & -6.26151 & Down & filamin A \\
\hline TTYH3 & -1.49872 & $3.77 \mathrm{E}-07$ & 0.000196 & -5.08044 & Down & tweety family member 3 \\
\hline RNU5B-1 & -1.48598 & 0.000606 & 0.020737 & -3.42908 & Down & RNA, U5B small nuclear 1 \\
\hline ACAD10 & -1.48224 & 0.000997 & 0.026839 & -3.29128 & Down & acyl-CoA dehydrogenase family member 10 \\
\hline TUBB1 & -1.4625 & $1.87 \mathrm{E}-05$ & 0.00255 & -4.27993 & Down & tubulin beta 1 class VI \\
\hline MTATP6P1 & -1.46162 & 0.000698 & 0.022616 & -3.39024 & Down & MT-ATP6 pseudogene 1 \\
\hline ATP2A3 & -1.45721 & $1.86 \mathrm{E}-07$ & 0.00012 & -5.21255 & Down & ATPase sarcoplasmic/endoplasmic reticulum $\mathrm{Ca} 2+$ transporting 3 \\
\hline BSCL2 & -1.45633 & 0.000547 & 0.019444 & -3.45651 & Down & BSCL2 lipid droplet biogenesis associated, seipin \\
\hline ARHGAP27 & -1.45245 & $3.89 \mathrm{E}-06$ & 0.000993 & -4.61721 & Down & Rho GTPase activating protein 27 \\
\hline TRPC6 & -1.44884 & 0.000582 & 0.020311 & -3.44006 & Down & transient receptor potential cation channel subfamily $\mathrm{C}$ member 6 \\
\hline SELP & -1.44569 & 7.27E-05 & 0.005584 & -3.96743 & Down & selectin P \\
\hline LMF2 & -1.44542 & 0.001479 & 0.033304 & -3.1787 & Down & lipase maturation factor 2 \\
\hline ZNF865 & -1.43756 & $2.84 \mathrm{E}-05$ & 0.003258 & -4.18559 & Down & zinc finger protein 865 \\
\hline SCARNA1 & -1.43102 & 0.002052 & 0.040029 & -3.08254 & Down & small Cajal body-specific RNA 1 \\
\hline CD79A & -1.43031 & $2.92 \mathrm{E}-06$ & 0.000822 & -4.67616 & Down & CD79a molecule \\
\hline CSF1R & -1.42263 & $1.30 \mathrm{E}-05$ & 0.001977 & -4.36033 & Down & colony stimulating factor 1 receptor \\
\hline TSPAN18 & -1.42096 & $8.69 \mathrm{E}-08$ & $8.60 \mathrm{E}-05$ & -5.3521 & Down & tetraspanin 18 \\
\hline MEMO1 & -1.41159 & 0.001686 & 0.035853 & -3.14057 & Down & mediator of cell motility 1 \\
\hline RAB27B & -1.4082 & $2.46 \mathrm{E}-05$ & 0.003077 & -4.21825 & Down & RAB27B, member RAS oncogene family \\
\hline MAP3K7CL & -1.39373 & 0.001733 & 0.036439 & -3.13253 & Down & MAP3K7 C-terminal like \\
\hline
\end{tabular}




\begin{tabular}{|c|c|c|c|c|c|c|}
\hline NUP210 & -1.39068 & $1.71 \mathrm{E}-05$ & 0.002402 & -4.29912 & Down & nucleoporin 210 \\
\hline XKR8 & -1.38657 & 0.001051 & 0.027608 & -3.27656 & Down & $\mathrm{XK}$ related 8 \\
\hline ZNF296 & -1.3763 & 0.000893 & 0.025475 & -3.32229 & Down & zinc finger protein 296 \\
\hline HLA-A & -1.36399 & $4.26 \mathrm{E}-05$ & 0.00411 & -4.09299 & Down & major histocompatibility complex, class I, A \\
\hline VCL & -1.35636 & $2.75 \mathrm{E}-06$ & 0.000789 & -4.68889 & Down & vinculin \\
\hline PYGB & -1.35046 & $1.37 \mathrm{E}-07$ & 0.000106 & -5.26879 & Down & glycogen phosphorylase B \\
\hline ZNF185 & -1.34888 & $7.28 \mathrm{E}-06$ & 0.001463 & -4.48526 & Down & zinc finger protein 185 with LIM domain \\
\hline TLN1 & -1.34869 & $9.33 \mathrm{E}-10$ & $2.29 \mathrm{E}-06$ & -6.1204 & Down & talin 1 \\
\hline ATG16L2 & -1.34232 & 0.000383 & 0.015563 & -3.55153 & Down & autophagy related 16 like 2 \\
\hline P2RY1 & -1.34195 & $6.23 \mathrm{E}-05$ & 0.005041 & -4.00391 & Down & purinergic receptor $\mathrm{P} 2 \mathrm{Y} 1$ \\
\hline FCRL1 & -1.33807 & 0.001187 & 0.029636 & -3.24197 & Down & Fc receptor like 1 \\
\hline VCAN & -1.33673 & $5.17 \mathrm{E}-06$ & 0.001218 & -4.5579 & Down & versican \\
\hline GP5 & -1.33529 & 0.000375 & 0.015331 & -3.55694 & Down & glycoprotein V platelet \\
\hline TREML1 & -1.32677 & 0.000354 & 0.014874 & -3.57241 & Down & triggering receptor expressed on myeloid cells like 1 \\
\hline ENDOD1 & -1.32673 & $3.35 \mathrm{E}-05$ & 0.003601 & -4.14836 & Down & endonuclease domain containing 1 \\
\hline SF3A2 & -1.32632 & 2.07E-05 & 0.002792 & -4.25694 & Down & splicing factor 3 a subunit 2 \\
\hline SNHG1 & -1.31645 & 3.57E-07 & 0.000191 & -5.09032 & Down & small nucleolar RNA host gene 1 \\
\hline CYBC1 & -1.31003 & $2.16 \mathrm{E}-06$ & 0.000683 & -4.73829 & Down & cytochrome b-245 chaperone 1 \\
\hline NOTCH2 & -1.30956 & $6.57 \mathrm{E}-06$ & 0.001388 & -4.50727 & Down & notch receptor 2 \\
\hline F2RL3 & -1.30319 & 0.000544 & 0.019385 & -3.4581 & Down & F2R like thrombin or trypsin receptor 3 \\
\hline MLEC & -1.30219 & $6.56 \mathrm{E}-05$ & 0.005188 & -3.99169 & Down & malectin \\
\hline LIPA & -1.30205 & $3.04 \mathrm{E}-06$ & 0.000836 & -4.66836 & Down & lipase A, lysosomal acid type \\
\hline PSAP & -1.29688 & $3.59 \mathrm{E}-09$ & $8.00 \mathrm{E}-06$ & -5.90214 & Down & prosaposin \\
\hline FAM185A & -1.29651 & 0.003014 & 0.048576 & -2.96634 & Down & family with sequence similarity 185 member A \\
\hline CHST12 & -1.29603 & 0.001818 & 0.037455 & -3.11853 & Down & carbohydrate sulfotransferase 12 \\
\hline ZNF142 & -1.28692 & 0.000461 & 0.017474 & -3.50237 & Down & zinc finger protein 142 \\
\hline $\mathrm{CD} 300 \mathrm{E}$ & -1.28208 & 4.95E-05 & 0.004447 & -4.05786 & Down & CD300e molecule \\
\hline LIMS1 & -1.27289 & $8.29 \mathrm{E}-06$ & 0.001552 & -4.45754 & Down & LIM zinc finger domain containing 1 \\
\hline MYBBP1A & -1.2726 & $1.15 \mathrm{E}-05$ & 0.001797 & -4.38768 & Down & MYB binding protein 1a \\
\hline TLR5 & -1.27091 & $3.23 \mathrm{E}-05$ & 0.003516 & -4.15682 & Down & toll like receptor 5 \\
\hline ACAA1 & -1.26927 & $5.32 \mathrm{E}-05$ & 0.00461 & -4.04102 & Down & acetyl-CoA acyltransferase 1 \\
\hline ABCA7 & -1.26175 & 0.000641 & 0.021466 & -3.41379 & Down & ATP binding cassette subfamily A member 7 \\
\hline ELAC2 & -1.2384 & 0.000275 & 0.012948 & -3.63763 & Down & elaC ribonuclease $\mathrm{Z} 2$ \\
\hline CAVIN2 & -1.23832 & 0.000225 & 0.011379 & -3.68867 & Down & caveolae associated protein 2 \\
\hline PTPRJ & -1.23748 & 7.34E-07 & 0.000321 & -4.9521 & Down & protein tyrosine phosphatase receptor type $\mathrm{J}$ \\
\hline MYCL & -1.23528 & 0.002527 & 0.044281 & -3.02011 & Down & MYCL proto-oncogene, bHLH transcription factor \\
\hline $\mathrm{ABCC} 3$ & -1.23485 & 0.000179 & 0.010131 & -3.74645 & Down & ATP binding cassette subfamily $\mathrm{C}$ member 3 \\
\hline LRP1 & -1.22373 & $2.68 \mathrm{E}-05$ & 0.003145 & -4.19944 & Down & LDL receptor related protein 1 \\
\hline $\mathrm{BAX}$ & -1.22016 & $3.69 \mathrm{E}-05$ & 0.00378 & -4.12579 & Down & BCL2 associated $\mathrm{X}$, apoptosis regulator \\
\hline SUN2 & -1.21453 & 0.001048 & 0.027588 & -3.27739 & Down & Sad1 and UNC84 domain containing 2 \\
\hline P2RX1 & -1.21224 & $8.63 \mathrm{E}-05$ & 0.006131 & -3.92616 & Down & purinergic receptor $\mathrm{P} 2 \mathrm{X} 1$ \\
\hline RNU4-2 & -1.20679 & 0.000666 & 0.021923 & -3.40305 & Down & RNA, U4 small nuclear 2 \\
\hline DENND1C & -1.20654 & $2.52 \mathrm{E}-05$ & 0.003077 & -4.21316 & Down & DENN domain containing 1C \\
\hline
\end{tabular}




\begin{tabular}{|c|c|c|c|c|c|c|}
\hline PYCR2 & -1.20379 & $2.51 \mathrm{E}-05$ & 0.003077 & -4.21399 & Down & pyrroline-5-carboxylate reductase 2 \\
\hline SMCR8 & -1.19691 & $7.53 \mathrm{E}-05$ & 0.005661 & -3.95896 & Down & SMCR8-C9orf72 complex subunit \\
\hline $\mathrm{ENO} 2$ & -1.19673 & $1.87 \mathrm{E}-05$ & 0.00255 & -4.27962 & Down & enolase 2 \\
\hline SPARC & -1.19573 & 0.000338 & 0.014421 & -3.58411 & Down & secreted protein acidic and cysteine rich \\
\hline TMEM140 & -1.19371 & 0.000156 & 0.009395 & -3.78076 & Down & transmembrane protein 140 \\
\hline CLU & -1.19052 & 0.000112 & 0.007242 & -3.86311 & Down & clusterin \\
\hline TBXA2R & -1.18747 & $3.50 \mathrm{E}-05$ & 0.003694 & -4.13853 & Down & thromboxane $\mathrm{A} 2$ receptor \\
\hline LRRC25 & -1.18687 & 0.000472 & 0.017694 & -3.49615 & Down & leucine rich repeat containing 25 \\
\hline CIITA & -1.18392 & 0.000112 & 0.007242 & -3.86311 & Down & class II major histocompatibility complex transactivator \\
\hline FCN1 & -1.18116 & $5.65 \mathrm{E}-06$ & 0.001284 & -4.5389 & Down & ficolin 1 \\
\hline NFRKB & -1.17991 & 0.001479 & 0.033304 & -3.17872 & Down & nuclear factor related to kappaB binding protein \\
\hline ITGB5 & -1.17791 & 0.000832 & 0.024307 & -3.34193 & Down & integrin subunit beta 5 \\
\hline PRKAR2B & -1.17746 & 0.000123 & 0.007728 & -3.84083 & Down & protein kinase cAMP-dependent type II regulatory subunit beta \\
\hline ITGA2 & -1.17733 & 0.001413 & 0.032461 & -3.19203 & Down & integrin subunit alpha 2 \\
\hline PPM1L & -1.17733 & 0.000627 & 0.021185 & -3.41987 & Down & protein phosphatase, $\mathrm{Mg} 2+/ \mathrm{Mn} 2+$ dependent $1 \mathrm{~L}$ \\
\hline CDKN1A & -1.17374 & 3.67E-05 & 0.00378 & -4.12762 & Down & cyclin dependent kinase inhibitor $1 \mathrm{~A}$ \\
\hline CCNL1 & -1.17343 & 0.000301 & 0.013571 & -3.61479 & Down & cyclin L1 \\
\hline MAP4K1 & -1.17148 & $3.68 \mathrm{E}-05$ & 0.00378 & -4.12662 & Down & mitogen-activated protein kinase kinase kinase kinase 1 \\
\hline ADCY7 & -1.16248 & $5.66 \mathrm{E}-05$ & 0.004783 & -4.02666 & Down & adenylate cyclase 7 \\
\hline TKFC & -1.15685 & 0.002084 & 0.040319 & -3.07803 & Down & triokinase and FMN cyclase \\
\hline ZC3HAV1L & -1.15209 & 0.002252 & 0.041643 & -3.05477 & Down & zinc finger $\mathrm{CCCH}$-type containing, antiviral 1 like \\
\hline MPEG1 & -1.15151 & $1.89 \mathrm{E}-06$ & 0.000616 & -4.76529 & Down & macrophage expressed 1 \\
\hline PANX1 & -1.14895 & 0.001235 & 0.030149 & -3.23074 & Down & pannexin 1 \\
\hline NRGN & -1.14792 & 0.000591 & 0.020472 & -3.43561 & Down & neurogranin \\
\hline RAB1B & -1.14574 & 3.37E-07 & 0.000184 & -5.10152 & Down & RAB1B, member RAS oncogene family \\
\hline CA5BP1 & -1.1382 & 0.001138 & 0.028918 & -3.25407 & Down & carbonic anhydrase 5B pseudogene 1 \\
\hline PDE5A & -1.13435 & 0.000138 & 0.00844 & -3.81219 & Down & phosphodiesterase $5 \mathrm{~A}$ \\
\hline CLPP & -1.12506 & $5.89 \mathrm{E}-05$ & 0.004918 & -4.01717 & Down & caseinolytic mitochondrial matrix peptidase proteolytic subunit \\
\hline NCSTN & -1.12446 & $4.82 \mathrm{E}-05$ & 0.004447 & -4.06399 & Down & nicastrin \\
\hline URB2 & -1.12263 & 0.000647 & 0.021519 & -3.41111 & Down & URB2 ribosome biogenesis homolog \\
\hline IRF7 & -1.12153 & 0.000197 & 0.010736 & -3.72219 & Down & interferon regulatory factor 7 \\
\hline CHERP & -1.11812 & 0.000256 & 0.012271 & -3.65642 & Down & calcium homeostasis endoplasmic reticulum protein \\
\hline SMAD3 & -1.11811 & 4.97E-05 & 0.004447 & -4.05702 & Down & SMAD family member 3 \\
\hline CTSW & -1.11737 & 0.000104 & 0.006936 & -3.88213 & Down & cathepsin W \\
\hline GLIPR1 & -1.11571 & 0.00141 & 0.03242 & -3.19266 & Down & GLI pathogenesis related 1 \\
\hline NIBAN3 & -1.11238 & 0.001211 & 0.029831 & -3.23632 & Down & niban apoptosis regulator 3 \\
\hline C2orf88 & -1.10948 & 0.001337 & 0.031528 & -3.20797 & Down & chromosome 2 open reading frame 88 \\
\hline CMIP & -1.10944 & $9.65 \mathrm{E}-11$ & $3.94 \mathrm{E}-07$ & -6.47226 & Down & c-Maf inducing protein \\
\hline SNHG3 & -1.10866 & 0.001906 & 0.038276 & -3.10448 & Down & small nucleolar RNA host gene 3 \\
\hline LFNG & -1.10857 & 0.000881 & 0.025232 & -3.32595 & Down & LFNG O-fucosylpeptide 3-beta-N-acetylglucosaminyltransferase \\
\hline TMEM259 & -1.10223 & 0.000105 & 0.006987 & -3.879 & Down & transmembrane protein 259 \\
\hline MFAP3L & -1.09951 & 0.000178 & 0.010116 & -3.74857 & Down & microfibril associated protein 3 like \\
\hline KDM2B & -1.09588 & 0.000316 & 0.013923 & -3.60202 & Down & lysine demethylase $2 \mathrm{~B}$ \\
\hline
\end{tabular}




\begin{tabular}{|c|c|c|c|c|c|c|}
\hline CTSA & -1.08776 & 0.000138 & 0.00844 & -3.81163 & Down & cathepsin A \\
\hline CMTM5 & -1.08502 & 0.000602 & 0.020717 & -3.43049 & Down & CKLF like MARVEL transmembrane domain containing 5 \\
\hline ITGB1 & -1.08493 & 0.000117 & 0.007479 & -3.85139 & Down & integrin subunit beta 1 \\
\hline TCL1A & -1.08285 & 0.000437 & 0.016891 & -3.51639 & Down & TCL1 family AKT coactivator A \\
\hline RHBDF2 & -1.0815 & 0.000644 & 0.021466 & -3.41243 & Down & rhomboid 5 homolog 2 \\
\hline MRPL28 & -1.07974 & 0.000739 & 0.023226 & -3.37468 & Down & mitochondrial ribosomal protein L28 \\
\hline DENND4B & -1.07669 & $4.36 \mathrm{E}-06$ & 0.001071 & -4.59333 & Down & DENN domain containing 4B \\
\hline PIK3IP1 & -1.0738 & 0.002438 & 0.04338 & -3.03089 & Down & phosphoinositide-3-kinase interacting protein 1 \\
\hline ANO6 & -1.0728 & 0.000183 & 0.010146 & -3.74208 & Down & $\operatorname{anoctamin} 6$ \\
\hline SIDT2 & -1.0724 & 0.001844 & 0.03779 & -3.11431 & Down & SID1 transmembrane family member 2 \\
\hline VPS52 & -1.07237 & 0.001962 & 0.039005 & -3.09597 & Down & VPS52 subunit of GARP complex \\
\hline CAPN1 & -1.07099 & $8.76 \mathrm{E}-08$ & $8.60 \mathrm{E}-05$ & -5.35063 & Down & calpain 1 \\
\hline BAK1 & -1.07031 & 0.002622 & 0.045039 & -3.00895 & Down & BCL2 antagonist/killer 1 \\
\hline IGHM & -1.06891 & $9.10 \mathrm{E}-06$ & 0.001665 & -4.43745 & Down & immunoglobulin heavy constant mu \\
\hline POLR1A & -1.06714 & 0.0005 & 0.018378 & -3.48087 & Down & RNA polymerase I subunit A \\
\hline NOTCH1 & -1.06581 & 0.000231 & 0.011467 & -3.68267 & Down & notch receptor 1 \\
\hline MS4A1 & -1.06515 & $8.76 \mathrm{E}-06$ & 0.001615 & -4.44574 & Down & membrane spanning 4-domains A1 \\
\hline PLEC & -1.06359 & $7.36 \mathrm{E}-06$ & 0.001466 & -4.48313 & Down & plectin \\
\hline HEATR6 & -1.06357 & 0.000833 & 0.024307 & -3.34158 & Down & HEAT repeat containing 6 \\
\hline SRPRA & -1.06195 & $3.45 \mathrm{E}-05$ & 0.00368 & -4.14139 & Down & SRP receptor subunit alpha \\
\hline FERMT3 & -1.05969 & 3.37E-06 & 0.000907 & -4.64715 & Down & fermitin family member 3 \\
\hline TLR2 & -1.05728 & 0.000587 & 0.020406 & -3.43764 & Down & toll like receptor 2 \\
\hline RNASET2 & -1.05719 & 0.001958 & 0.039005 & -3.09646 & Down & ribonuclease $\mathrm{T} 2$ \\
\hline GSE1 & -1.05705 & 0.000165 & 0.009631 & -3.76795 & Down & Gse1 coiled-coil protein \\
\hline DNAH1 & -1.05642 & 0.002558 & 0.044661 & -3.01643 & Down & dynein axonemal heavy chain 1 \\
\hline GPR183 & -1.05545 & 0.001287 & 0.030786 & -3.21883 & Down & G protein-coupled receptor 183 \\
\hline TCIRG1 & -1.05428 & 0.000767 & 0.023535 & -3.36442 & Down & $\mathrm{T}$ cell immune regulator 1 , ATPase $\mathrm{H}+$ transporting V0 subunit a3 \\
\hline PLXDC2 & -1.05364 & $1.13 \mathrm{E}-05$ & 0.001796 & -4.39103 & Down & plexin domain containing 2 \\
\hline ESYT1 & -1.05169 & 0.000207 & 0.011081 & -3.71086 & Down & extended synaptotagmin 1 \\
\hline LOC728975 & -1.05112 & 0.000525 & 0.018855 & -3.46791 & Down & uncharacterized LOC728975 \\
\hline GIGYF1 & -1.04994 & $9.89 \mathrm{E}-05$ & 0.006716 & -3.89329 & Down & GRB10 interacting GYF protein 1 \\
\hline HLA-E & -1.04792 & 7.06E-06 & 0.001447 & -4.49174 & Down & major histocompatibility complex, class I, E \\
\hline TPP1 & -1.03673 & 7.65E-05 & 0.005698 & -3.95523 & Down & tripeptidyl peptidase 1 \\
\hline C1orf198 & -1.03507 & 0.00033 & 0.0142 & -3.59083 & Down & chromosome 1 open reading frame 198 \\
\hline LENG8 & -1.03282 & 0.00077 & 0.023545 & -3.36347 & Down & leukocyte receptor cluster member 8 \\
\hline MMD & -1.03273 & 0.001966 & 0.039069 & -3.09525 & Down & monocyte to macrophage differentiation associated \\
\hline ARHGAP6 & -1.03147 & $9.83 \mathrm{E}-05$ & 0.006697 & -3.89486 & Down & Rho GTPase activating protein 6 \\
\hline INF2 & -1.03092 & 4.89E-05 & 0.004447 & -4.06078 & Down & inverted formin 2 \\
\hline $\mathrm{ADA} 2$ & -1.03056 & $3.22 \mathrm{E}-05$ & 0.003516 & -4.15714 & Down & adenosine deaminase 2 \\
\hline LEPROT & -1.02826 & 0.000362 & 0.015 & -3.56638 & Down & leptin receptor overlapping transcript \\
\hline LHFPL2 & -1.02583 & 0.000168 & 0.009794 & -3.76256 & Down & LHFPL tetraspan subfamily member 2 \\
\hline RSRP1 & -1.02528 & 0.00013 & 0.008082 & -3.82605 & Down & arginine and serine rich protein 1 \\
\hline PRR12 & -1.02355 & $5.89 \mathrm{E}-05$ & 0.004918 & -4.01726 & Down & proline rich 12 \\
\hline
\end{tabular}




\begin{tabular}{|c|c|c|c|c|c|c|}
\hline PTPRC & -1.02342 & $6.41 \mathrm{E}-06$ & 0.001381 & -4.51254 & Down & protein tyrosine phosphatase receptor type $\mathrm{C}$ \\
\hline PEAR1 & -1.02315 & 0.000976 & 0.026694 & -3.29731 & Down & platelet endothelial aggregation receptor 1 \\
\hline SH3BP1 & -1.02067 & 0.000164 & 0.009631 & -3.76841 & Down & SH3 domain binding protein 1 \\
\hline CBFA2T3 & -1.01554 & 4.37E-06 & 0.001071 & -4.59298 & Down & CBFA2/RUNX1 partner transcriptional co-repressor 3 \\
\hline SH3BGRL2 & -1.01405 & 0.001013 & 0.027022 & -3.28691 & Down & SH3 domain binding glutamate rich protein like 2 \\
\hline NBEAL2 & -1.01302 & $1.66 \mathrm{E}-07$ & 0.000114 & -5.23428 & Down & neurobeachin like 2 \\
\hline USP20 & -1.01201 & 0.000312 & 0.013829 & -3.60538 & Down & ubiquitin specific peptidase 20 \\
\hline ACTN1 & -1.01061 & $1.38 \mathrm{E}-07$ & 0.000106 & -5.26824 & Down & actinin alpha 1 \\
\hline GALNT10 & -1.01018 & $6.48 \mathrm{E}-05$ & 0.005161 & -3.99445 & Down & polypeptide $\mathrm{N}$-acetylgalactosaminyltransferase 10 \\
\hline MYH9 & -1.00874 & $3.06 \mathrm{E}-08$ & 4.68E-05 & -5.53822 & Down & myosin heavy chain 9 \\
\hline EML3 & -1.00631 & 0.001341 & 0.031529 & -3.20698 & Down & EMAP like 3 \\
\hline CHPF2 & -1.00208 & 0.003133 & 0.049553 & -2.95433 & Down & chondroitin polymerizing factor 2 \\
\hline OGT & -0.99967 & $6.23 \mathrm{E}-06$ & 0.001364 & -4.51837 & Down & O-linked N-acetylglucosamine (GlcNAc) transferase \\
\hline TGFB1 & -0.99647 & $9.73 \mathrm{E}-06$ & 0.001712 & -4.42309 & Down & transforming growth factor beta 1 \\
\hline SLC35E2B & -0.99475 & 0.002332 & 0.042463 & -3.04436 & Down & solute carrier family 35 member E2B \\
\hline ITGA4 & -0.99164 & $1.11 \mathrm{E}-05$ & 0.001787 & -4.39552 & Down & integrin subunit alpha 4 \\
\hline SLA2 & -0.99112 & $1.58 \mathrm{E}-05$ & 0.002264 & -4.31754 & Down & Src like adaptor 2 \\
\hline PITPNM2 & -0.99094 & 0.000218 & 0.011333 & -3.69702 & Down & phosphatidylinositol transfer protein membrane associated 2 \\
\hline DTX1 & -0.99043 & 0.000227 & 0.011442 & -3.68656 & Down & deltex E3 ubiquitin ligase 1 \\
\hline CARD11 & -0.98965 & 0.000414 & 0.016355 & -3.53081 & Down & caspase recruitment domain family member 11 \\
\hline MADD & -0.98635 & 8.67E-08 & $8.60 \mathrm{E}-05$ & -5.35256 & Down & MAP kinase activating death domain \\
\hline KCNA3 & -0.98406 & 0.000815 & 0.024176 & -3.34766 & Down & potassium voltage-gated channel subfamily A member 3 \\
\hline MRTFA & -0.98003 & 7.14E-06 & 0.001447 & -4.48947 & Down & myocardin related transcription factor A \\
\hline EHD3 & -0.97891 & $1.58 \mathrm{E}-05$ & 0.002264 & -4.31736 & Down & EH domain containing 3 \\
\hline JAK3 & -0.97683 & $2.29 \mathrm{E}-06$ & 0.000692 & -4.72564 & Down & Janus kinase 3 \\
\hline SMG5 & -0.97651 & $3.42 \mathrm{E}-05$ & 0.003666 & -4.14321 & Down & SMG5 nonsense mediated mRNA decay factor \\
\hline BTLA & -0.97428 & 0.000977 & 0.026694 & -3.29714 & Down & B and T lymphocyte associated \\
\hline ITM2B & -0.97395 & 0.000939 & 0.026167 & -3.30808 & Down & integral membrane protein $2 \mathrm{~B}$ \\
\hline SRC & -0.96844 & $6.51 \mathrm{E}-05$ & 0.005166 & -3.99349 & Down & SRC proto-oncogene, non-receptor tyrosine kinase \\
\hline DSE & -0.96802 & 0.001176 & 0.029554 & -3.24454 & Down & dermatan sulfate epimerase \\
\hline GP9 & -0.96762 & 0.001032 & 0.02733 & -3.28158 & Down & glycoprotein IX platelet \\
\hline APP & -0.96469 & 0.000121 & 0.007643 & -3.84417 & Down & amyloid beta precursor protein \\
\hline PCSK6 & -0.96413 & 0.00118 & 0.029566 & -3.24363 & Down & proprotein convertase subtilisin/kexin type 6 \\
\hline FGD3 & -0.96372 & $5.96 \mathrm{E}-05$ & 0.00493 & -4.01439 & Down & FYVE, RhoGEF and PH domain containing 3 \\
\hline TMEM63A & -0.96257 & 0.000766 & 0.023535 & -3.36466 & Down & transmembrane protein $63 \mathrm{~A}$ \\
\hline UNC13D & -0.96254 & 4.23E-07 & 0.000207 & -5.0585 & Down & unc-13 homolog D \\
\hline POU2F2 & -0.96008 & $5.20 \mathrm{E}-05$ & 0.004533 & -4.04663 & Down & POU class 2 homeobox 2 \\
\hline TGOLN2 & -0.95956 & $1.07 \mathrm{E}-07$ & $9.13 \mathrm{E}-05$ & -5.31445 & Down & trans-golgi network protein 2 \\
\hline ALOX12 & -0.95833 & 0.002466 & 0.043601 & -3.02754 & Down & arachidonate 12 -lipoxygenase, $12 \mathrm{~S}$ type \\
\hline FHOD1 & -0.95699 & 0.000725 & 0.022988 & -3.38001 & Down & formin homology 2 domain containing 1 \\
\hline TMC6 & -0.95675 & 0.000506 & 0.018497 & -3.47745 & Down & transmembrane channel like 6 \\
\hline MAP1A & -0.95616 & 0.001254 & 0.030269 & -3.22621 & Down & microtubule associated protein $1 \mathrm{~A}$ \\
\hline STIM1 & -0.956 & 0.000171 & 0.009894 & -3.75824 & Down & stromal interaction molecule 1 \\
\hline
\end{tabular}




\begin{tabular}{|c|c|c|c|c|c|c|}
\hline CTDSPL & -0.95589 & 3.63E-05 & 0.00378 & -4.1296 & Down & CTD small phosphatase like \\
\hline NHSL2 & -0.95568 & 0.000407 & 0.016188 & -3.53524 & Down & NHS like 2 \\
\hline FTSJ1 & -0.9548 & 0.000213 & 0.011232 & -3.70358 & Down & FtsJ RNA 2'-O-methyltransferase 1 \\
\hline RAB5C & -0.95199 & 0.000674 & 0.022111 & -3.40002 & Down & RAB5C, member RAS oncogene family \\
\hline $\mathrm{ZYX}$ & -0.95107 & $2.11 \mathrm{E}-07$ & 0.000133 & -5.18909 & Down & zyxin \\
\hline GUCY1B1 & -0.95093 & 0.000202 & 0.010901 & -3.71667 & Down & guanylate cyclase 1 soluble subunit beta 1 \\
\hline СMTM3 & -0.95034 & 0.001706 & 0.036147 & -3.13716 & Down & CKLF like MARVEL transmembrane domain containing 3 \\
\hline CNST & -0.94803 & 0.000133 & 0.008228 & -3.82102 & Down & consortin, connexin sorting protein \\
\hline ITPR1 & -0.94779 & $9.85 \mathrm{E}-06$ & 0.001712 & -4.42051 & Down & inositol 1,4,5-trisphosphate receptor type 1 \\
\hline GANAB & -0.94722 & $2.41 \mathrm{E}-05$ & 0.003076 & -4.22326 & Down & glucosidase II alpha subunit \\
\hline LDLRAP1 & -0.94622 & $7.85 \mathrm{E}-06$ & 0.00154 & -4.46915 & Down & low density lipoprotein receptor adaptor protein 1 \\
\hline CCNL2 & -0.94337 & 0.001671 & 0.035724 & -3.14316 & Down & cyclin L2 \\
\hline SCAF1 & -0.94322 & 0.000444 & 0.017101 & -3.51224 & Down & SR-related CTD associated factor 1 \\
\hline RNF213 & -0.94236 & $9.61 \mathrm{E}-07$ & 0.000386 & -4.89945 & Down & ring finger protein 213 \\
\hline MYLK & -0.93801 & 0.000244 & 0.011968 & -3.66838 & Down & myosin light chain kinase \\
\hline CENPT & -0.93691 & 0.000923 & 0.025996 & -3.31315 & Down & centromere protein $\mathrm{T}$ \\
\hline ARL15 & -0.93608 & 0.001004 & 0.02689 & -3.28952 & Down & ADP ribosylation factor like GTPase 15 \\
\hline SRSF6 & -0.93462 & $2.87 \mathrm{E}-05$ & 0.003258 & -4.18353 & Down & serine and arginine rich splicing factor 6 \\
\hline LILRB2 & -0.93243 & 0.001756 & 0.036694 & -3.12872 & Down & leukocyte immunoglobulin like receptor B2 \\
\hline TYMP & -0.92831 & 0.000169 & 0.009825 & -3.76117 & Down & thymidine phosphorylase \\
\hline EPM2AIP1 & -0.9279 & 0.000549 & 0.019464 & -3.45543 & Down & EPM2A interacting protein 1 \\
\hline SELENON & -0.92751 & 0.000614 & 0.020887 & -3.42524 & Down & selenoprotein $\mathrm{N}$ \\
\hline SNRPA & -0.92596 & 0.000312 & 0.013829 & -3.60519 & Down & small nuclear ribonucleoprotein polypeptide A \\
\hline RUFY1 & -0.92552 & 0.001177 & 0.029554 & -3.2445 & Down & RUN and FYVE domain containing 1 \\
\hline GNB5 & -0.92404 & 0.002044 & 0.039991 & -3.08379 & Down & $\mathrm{G}$ protein subunit beta 5 \\
\hline PRUNE1 & -0.92163 & $6.17 \mathrm{E}-05$ & 0.005023 & -4.00634 & Down & prune exopolyphosphatase 1 \\
\hline HELZ2 & -0.92021 & 0.001894 & 0.038276 & -3.10633 & Down & helicase with zinc finger 2 \\
\hline GPR137B & -0.91873 & 0.000789 & 0.023846 & -3.35671 & Down & G protein-coupled receptor 137B \\
\hline MBD6 & -0.91809 & 0.001696 & 0.035973 & -3.13883 & Down & methyl-CpG binding domain protein 6 \\
\hline MTPN & -0.91698 & 0.000107 & 0.007035 & -3.87486 & Down & myotrophin \\
\hline PDGFA & -0.91698 & 0.000286 & 0.013232 & -3.62811 & Down & platelet derived growth factor subunit A \\
\hline ITGB2 & -0.91566 & $9.79 \mathrm{E}-06$ & 0.001712 & -4.42169 & Down & integrin subunit beta 2 \\
\hline METTL26 & -0.91492 & 0.000816 & 0.024176 & -3.34723 & Down & methyltransferase like 26 \\
\hline SELPLG & -0.91412 & 0.000977 & 0.026694 & -3.29704 & Down & selectin P ligand \\
\hline АТР9A & -0.91117 & 0.00249 & 0.043911 & -3.0246 & Down & ATPase phospholipid transporting 9A (putative) \\
\hline DAPP1 & -0.90904 & 0.00144 & 0.032813 & -3.18648 & Down & dual adaptor of phosphotyrosine and 3-phosphoinositides 1 \\
\hline CORO1B & -0.90571 & $1.07 \mathrm{E}-05$ & 0.001778 & -4.40295 & Down & coronin $1 \mathrm{~B}$ \\
\hline TRIM13 & -0.90533 & $8.14 \mathrm{E}-06$ & 0.001552 & -4.46152 & Down & tripartite motif containing 13 \\
\hline SIN3B & -0.90521 & 0.002163 & 0.041072 & -3.06691 & Down & SIN3 transcription regulator family member B \\
\hline VPS37B & -0.90416 & $2.95 \mathrm{E}-06$ & 0.000822 & -4.67423 & Down & VPS37B subunit of ESCRT-I \\
\hline UPF1 & -0.9016 & $3.35 \mathrm{E}-08$ & 4.69E-05 & -5.52211 & Down & UPF1 RNA helicase and ATPase \\
\hline GDI1 & -0.90075 & $1.32 \mathrm{E}-06$ & 0.000476 & -4.83669 & Down & GDP dissociation inhibitor 1 \\
\hline TNFSF4 & -0.89928 & 0.002323 & 0.042463 & -3.04544 & Down & TNF superfamily member 4 \\
\hline
\end{tabular}




\begin{tabular}{|c|c|c|c|c|c|c|}
\hline PGAP6 & -0.89926 & 0.000729 & 0.023053 & -3.37853 & Down & post-glycosylphosphatidylinositol attachment to proteins 6 \\
\hline DIMT1 & -0.89763 & $8.41 \mathrm{E}-05$ & 0.006062 & -3.93238 & Down & DIMT1 rRNA methyltransferase and ribosome maturation factor \\
\hline RPA1 & -0.89722 & 0.001813 & 0.037437 & -3.11934 & Down & replication protein $\mathrm{A} 1$ \\
\hline RAB37 & -0.89716 & 0.000134 & 0.008296 & -3.81837 & Down & RAB37, member RAS oncogene family \\
\hline SPN & -0.89706 & 0.000109 & 0.007142 & -3.87038 & Down & sialophorin \\
\hline BCL11B & -0.89634 & 0.00012 & 0.007579 & -3.84685 & Down & BAF chromatin remodeling complex subunit BCL11B \\
\hline IMPDH1 & -0.89595 & $1.63 \mathrm{E}-05$ & 0.00233 & -4.30975 & Down & inosine monophosphate dehydrogenase 1 \\
\hline ZBTB7B & -0.89564 & $1.11 \mathrm{E}-05$ & 0.001787 & -4.39371 & Down & zinc finger and BTB domain containing 7B \\
\hline UBA7 & -0.89534 & 0.000336 & 0.014363 & -3.58607 & Down & ubiquitin like modifier activating enzyme 7 \\
\hline IL17RA & -0.89386 & 0.002731 & 0.045957 & -2.99647 & Down & interleukin 17 receptor $\mathrm{A}$ \\
\hline ARHGAP45 & -0.89323 & 1.19E-06 & 0.000443 & -4.85691 & Down & Rho GTPase activating protein 45 \\
\hline CBX6 & -0.89155 & 7.04E-05 & 0.005444 & -3.97496 & Down & chromobox 6 \\
\hline FAM78A & -0.89123 & $6.04 \mathrm{E}-05$ & 0.004955 & -4.0111 & Down & family with sequence similarity 78 member A \\
\hline IL10RA & -0.89088 & 0.00059 & 0.020458 & -3.43619 & Down & interleukin 10 receptor subunit alpha \\
\hline $\mathrm{CCDC} 88 \mathrm{~B}$ & -0.8901 & 0.000749 & 0.023336 & -3.37094 & Down & coiled-coil domain containing $88 \mathrm{~B}$ \\
\hline FGD2 & -0.887 & 0.000318 & 0.013947 & -3.60058 & Down & FYVE, RhoGEF and PH domain containing 2 \\
\hline IL6R & -0.88694 & 0.002874 & 0.04719 & -2.98092 & Down & interleukin 6 receptor \\
\hline TIMP1 & -0.88474 & 0.002332 & 0.042463 & -3.04433 & Down & TIMP metallopeptidase inhibitor 1 \\
\hline CST3 & -0.88244 & 4.94E-05 & 0.004447 & -4.05853 & Down & cystatin $\mathrm{C}$ \\
\hline ARHGEF18 & -0.88146 & 0.001 & 0.026845 & -3.2906 & Down & Rho/Rac guanine nucleotide exchange factor 18 \\
\hline SUSD3 & -0.87991 & 0.002546 & 0.044513 & -3.0178 & Down & sushi domain containing 3 \\
\hline FPGT & -0.87627 & 0.001177 & 0.029554 & -3.24451 & Down & fucose-1-phosphate guanylyltransferase \\
\hline TSPAN14 & -0.87607 & $1.01 \mathrm{E}-06$ & 0.000401 & -4.88878 & Down & tetraspanin 14 \\
\hline BCL3 & -0.87565 & 0.000821 & 0.024176 & -3.34553 & Down & BCL3 transcription coactivator \\
\hline CASP6 & -0.87492 & 0.001961 & 0.039005 & -3.0961 & Down & caspase 6 \\
\hline TUBA4A & -0.87399 & $8.71 \mathrm{E}-05$ & 0.006153 & -3.92402 & Down & tubulin alpha $4 \mathrm{a}$ \\
\hline STON2 & -0.87283 & 0.000357 & 0.014946 & -3.57025 & Down & stonin 2 \\
\hline GAS2L1 & -0.8701 & 0.002448 & 0.043475 & -3.02971 & Down & growth arrest specific 2 like 1 \\
\hline PRAM1 & -0.86941 & 0.000909 & 0.025759 & -3.31732 & Down & PML-RARA regulated adaptor molecule 1 \\
\hline DOCK8 & -0.86409 & 3.44E-08 & 4.69E-05 & -5.51723 & Down & dedicator of cytokinesis 8 \\
\hline FAM117B & -0.86342 & $2.68 \mathrm{E}-05$ & 0.003145 & -4.19897 & Down & family with sequence similarity 117 member B \\
\hline GRAP2 & -0.86129 & 0.001287 & 0.030786 & -3.21895 & Down & GRB2 related adaptor protein 2 \\
\hline C15orf39 & -0.85788 & $8.10 \mathrm{E}-05$ & 0.005983 & -3.94135 & Down & chromosome 15 open reading frame 39 \\
\hline DGKA & -0.85734 & 0.003058 & 0.048926 & -2.96185 & Down & diacylglycerol kinase alpha \\
\hline ARHGEF1 & -0.85684 & 4.39E-05 & 0.004178 & -4.08616 & Down & Rho guanine nucleotide exchange factor 1 \\
\hline RBM15 & -0.85623 & 0.000861 & 0.02485 & -3.33242 & Down & RNA binding motif protein 15 \\
\hline EVL & -0.85591 & 2.24E-07 & 0.000137 & -5.17847 & Down & Enah/Vasp-like \\
\hline ARL6IP5 & -0.85568 & 0.000964 & 0.026542 & -3.30096 & Down & ADP ribosylation factor like GTPase 6 interacting protein 5 \\
\hline LRBA & -0.85445 & 0.000194 & 0.01064 & -3.72615 & Down & LPS responsive beige-like anchor protein \\
\hline IL2RG & -0.85243 & 0.000449 & 0.017213 & -3.50955 & Down & interleukin 2 receptor subunit gamma \\
\hline PACSIN2 & -0.85239 & $1.42 \mathrm{E}-05$ & 0.002103 & -4.3404 & Down & protein kinase $\mathrm{C}$ and casein kinase substrate in neurons 2 \\
\hline MGAT4B & -0.85231 & 0.000371 & 0.015208 & -3.55994 & Down & alpha-1,3-mannosyl-glycoprotein 4-beta-N-acetylglucosaminyltransferase B \\
\hline TNFRSF1B & -0.85034 & 0.000653 & 0.021673 & -3.40846 & Down & TNF receptor superfamily member $1 \mathrm{~B}$ \\
\hline
\end{tabular}




\begin{tabular}{|c|c|c|c|c|c|c|}
\hline LIMD2 & -0.84822 & $9.25 \mathrm{E}-06$ & 0.001667 & -4.4341 & Down & LIM domain containing 2 \\
\hline FCMR & -0.84735 & 0.002891 & 0.047414 & -2.97906 & Down & Fc fragment of IgM receptor \\
\hline MOB1B & -0.84594 & 0.001353 & 0.031558 & -3.20455 & Down & MOB kinase activator $1 \mathrm{~B}$ \\
\hline MEPCE & -0.84434 & 0.000598 & 0.020599 & -3.4324 & Down & methylphosphate capping enzyme \\
\hline SERPINA1 & -0.8439 & $9.42 \mathrm{E}-06$ & 0.001685 & -4.43015 & Down & serpin family A member 1 \\
\hline PARVB & -0.84297 & 0.000128 & 0.007983 & -3.83009 & Down & parvin beta \\
\hline CYTH4 & -0.83743 & 0.000329 & 0.0142 & -3.59133 & Down & cytohesin 4 \\
\hline MINK1 & -0.83717 & $1.35 \mathrm{E}-05$ & 0.00202 & -4.35162 & Down & misshapen like kinase 1 \\
\hline CDK9 & -0.83717 & 0.002799 & 0.046486 & -2.98902 & Down & cyclin dependent kinase 9 \\
\hline RNF166 & -0.83615 & 7.52E-05 & 0.005661 & -3.95934 & Down & ring finger protein 166 \\
\hline BACH2 & -0.83557 & 0.000479 & 0.017835 & -3.49213 & Down & BTB domain and CNC homolog 2 \\
\hline C16orf54 & -0.83517 & 0.000303 & 0.013583 & -3.61268 & Down & chromosome 16 open reading frame 54 \\
\hline ST6GAL1 & -0.83265 & $5.76 \mathrm{E}-06$ & 0.001296 & -4.53494 & Down & ST6 beta-galactoside alpha-2,6-sialyltransferase 1 \\
\hline PIK3CD & -0.83256 & $2.41 \mathrm{E}-05$ & 0.003076 & -4.22314 & Down & phosphatidylinositol-4,5-bisphosphate 3-kinase catalytic subunit delta \\
\hline $\mathrm{CD} 84$ & -0.83207 & 0.000997 & 0.026839 & -3.29142 & Down & CD84 molecule \\
\hline PIGS & -0.83204 & 0.00231 & 0.042348 & -3.04724 & Down & phosphatidylinositol glycan anchor biosynthesis class $\mathrm{S}$ \\
\hline PAX5 & -0.83198 & 0.002781 & 0.046321 & -2.99094 & Down & paired box 5 \\
\hline SLC12A6 & -0.83191 & 0.000811 & 0.024176 & -3.34888 & Down & solute carrier family 12 member 6 \\
\hline TSC22D3 & -0.83029 & 1.19E-05 & 0.001852 & -4.37871 & Down & TSC22 domain family member 3 \\
\hline PI4KA & -0.82863 & 2.97E-05 & 0.003326 & -4.17553 & Down & phosphatidylinositol 4-kinase alpha \\
\hline SLC2A3 & -0.82562 & 0.000214 & 0.011264 & -3.70149 & Down & solute carrier family 2 member 3 \\
\hline CDK2AP1 & -0.82435 & 0.002237 & 0.041485 & -3.05681 & Down & cyclin dependent kinase 2 associated protein 1 \\
\hline RNF167 & -0.82309 & $9.29 \mathrm{E}-05$ & 0.006395 & -3.90851 & Down & ring finger protein 167 \\
\hline CXCR4 & -0.82239 & 0.000939 & 0.026167 & -3.30808 & Down & $\mathrm{C}-\mathrm{X}-\mathrm{C}$ motif chemokine receptor 4 \\
\hline ITGAL & -0.82201 & $8.43 \mathrm{E}-05$ & 0.006062 & -3.9319 & Down & integrin subunit alpha $\mathrm{L}$ \\
\hline TESPA1 & -0.82069 & 0.001242 & 0.030213 & -3.229 & Down & thymocyte expressed, positive selection associated 1 \\
\hline ZNF641 & -0.8206 & 0.00135 & 0.031558 & -3.20511 & Down & zinc finger protein 641 \\
\hline AFF3 & -0.82016 & 0.000985 & 0.026772 & -3.2948 & Down & AF4/FMR2 family member 3 \\
\hline MAP4K2 & -0.82004 & 0.000221 & 0.011379 & -3.69405 & Down & mitogen-activated protein kinase kinase kinase kinase 2 \\
\hline RNPEPL1 & -0.81798 & $3.53 \mathrm{E}-06$ & 0.00094 & -4.63733 & Down & arginyl aminopeptidase like 1 \\
\hline TNRC18 & -0.81713 & 0.001432 & 0.032712 & -3.18818 & Down & trinucleotide repeat containing 18 \\
\hline TMEM64 & -0.81185 & 0.0011 & 0.028265 & -3.26365 & Down & transmembrane protein 64 \\
\hline CTTN & -0.81143 & 0.000508 & 0.018519 & -3.4767 & Down & cortactin \\
\hline $\mathrm{CD} 47$ & -0.81045 & 0.000471 & 0.017682 & -3.49677 & Down & CD47 molecule \\
\hline $\mathrm{ABI} 3$ & -0.80921 & 0.003 & 0.048449 & -2.96775 & Down & ABI family member 3 \\
\hline NUP188 & -0.80778 & 0.0019 & 0.038276 & -3.10549 & Down & nucleoporin 188 \\
\hline DOK2 & -0.80762 & $8.22 \mathrm{E}-05$ & 0.00603 & -3.93804 & Down & docking protein 2 \\
\hline JADE2 & -0.80698 & $9.22 \mathrm{E}-05$ & 0.006385 & -3.91037 & Down & jade family PHD finger 2 \\
\hline APLP2 & -0.80381 & 0.000383 & 0.015563 & -3.55124 & Down & amyloid beta precursor like protein 2 \\
\hline PRRC2B & -0.80096 & 4.09E-07 & 0.000205 & -5.06466 & Down & proline rich coiled-coil 2B \\
\hline RNH1 & -0.80085 & 0.000149 & 0.009015 & -3.79346 & Down & ribonuclease/angiogenin inhibitor 1 \\
\hline ITGAM & -0.79865 & $1.15 \mathrm{E}-05$ & 0.001797 & -4.38772 & Down & integrin subunit alpha $\mathrm{M}$ \\
\hline FBRSL1 & -0.79664 & 0.00099 & 0.026772 & -3.29346 & Down & fibrosin like 1 \\
\hline
\end{tabular}




\begin{tabular}{|c|c|c|c|c|c|c|}
\hline DAD1 & -0.7966 & 0.002406 & 0.043137 & -3.03492 & Down & defender against cell death 1 \\
\hline FTH1 & -0.79596 & 0.002446 & 0.043475 & -3.02996 & Down & ferritin heavy chain 1 \\
\hline WAS & -0.79575 & $2.44 \mathrm{E}-05$ & 0.003077 & -4.22002 & Down & WASP actin nucleation promoting factor \\
\hline PKM & -0.79409 & 4.67E-05 & 0.004372 & -4.07146 & Down & $\begin{array}{l}\text { pyruvate kinase M1/2 } \\
\text { microtubule associated monooxygenase, calponin and LIM domain }\end{array}$ \\
\hline MICAL1 & -0.79024 & 0.001644 & 0.035387 & -3.14798 & Down & containing 1 \\
\hline MED12 & -0.78919 & 0.000644 & 0.021466 & -3.41225 & Down & mediator complex subunit 12 \\
\hline RASGRP2 & -0.78853 & $3.55 \mathrm{E}-05$ & 0.003732 & -4.13517 & Down & RAS guanyl releasing protein 2 \\
\hline CHST15 & -0.78715 & 0.001528 & 0.033794 & -3.16925 & Down & carbohydrate sulfotransferase 15 \\
\hline ZNF407 & -0.78667 & 0.00052 & 0.018756 & -3.47011 & Down & zinc finger protein 407 \\
\hline TTC7A & -0.78576 & 0.000256 & 0.012271 & -3.65643 & Down & tetratricopeptide repeat domain 7A \\
\hline NKTR & -0.78507 & 0.002233 & 0.041471 & -3.05737 & Down & natural killer cell triggering receptor \\
\hline RNF44 & -0.78352 & $2.18 \mathrm{E}-06$ & 0.000683 & -4.73584 & Down & ring finger protein 44 \\
\hline TNIK & -0.7832 & 4.96E-05 & 0.004447 & -4.05744 & Down & TRAF2 and NCK interacting kinase \\
\hline ACTR8 & -0.78285 & 0.001681 & 0.035795 & -3.1415 & Down & actin related protein 8 \\
\hline ITM2A & -0.78053 & 0.002761 & 0.046261 & -2.9932 & Down & integral membrane protein $2 \mathrm{~A}$ \\
\hline CD74 & -0.78038 & $1.23 \mathrm{E}-06$ & 0.000451 & -4.85041 & Down & CD74 molecule \\
\hline TMEM159 & -0.77781 & 0.002626 & 0.045057 & -3.00842 & Down & transmembrane protein 159 \\
\hline WDFY4 & -0.77731 & 0.000189 & 0.010417 & -3.73316 & Down & WDFY family member 4 \\
\hline MINDY1 & -0.77692 & 0.001399 & 0.032282 & -3.19489 & Down & MINDY lysine 48 deubiquitinase 1 \\
\hline SETD1B & -0.77688 & 0.001049 & 0.027588 & -3.27716 & Down & SET domain containing $1 \mathrm{~B}$, histone lysine methyltransferase \\
\hline STRN4 & -0.7768 & 0.00041 & 0.016205 & -3.53368 & Down & striatin 4 \\
\hline ARHGAP18 & -0.77625 & 0.002128 & 0.040759 & -3.07176 & Down & Rho GTPase activating protein 18 \\
\hline CSF3R & -0.77608 & $5.45 \mathrm{E}-05$ & 0.004668 & -4.03564 & Down & colony stimulating factor 3 receptor \\
\hline MTHFR & -0.77535 & 0.002156 & 0.041002 & -3.06789 & Down & methylenetetrahydrofolate reductase \\
\hline KLF2 & -0.77468 & 1.76E-06 & 0.000606 & -4.77956 & Down & Kruppel like factor 2 \\
\hline MYO1G & -0.77353 & 0.000326 & 0.014131 & -3.59398 & Down & myosin IG \\
\hline MBNL1 & -0.77351 & 0.00024 & 0.01188 & -3.67283 & Down & muscleblind like splicing regulator 1 \\
\hline CHMP1A & -0.77317 & 0.002612 & 0.044998 & -3.01007 & Down & charged multivesicular body protein $1 \mathrm{~A}$ \\
\hline TMEM40 & -0.77232 & 0.002399 & 0.043101 & -3.03585 & Down & transmembrane protein 40 \\
\hline TSC22D4 & -0.77095 & 0.000528 & 0.018925 & -3.46615 & Down & TSC22 domain family member 4 \\
\hline VPS51 & -0.77093 & 0.000908 & 0.025753 & -3.3177 & Down & VPS51 subunit of GARP complex \\
\hline PRKCA & -0.77022 & 0.000918 & 0.025903 & -3.31447 & Down & protein kinase $\mathrm{C}$ alpha \\
\hline FOXK1 & -0.7701 & $6.94 \mathrm{E}-05$ & 0.005383 & -3.97838 & Down & forkhead box K1 \\
\hline STXBP2 & -0.76972 & 0.000203 & 0.010914 & -3.7158 & Down & syntaxin binding protein 2 \\
\hline MYADM & -0.76451 & 0.001184 & 0.029614 & -3.24264 & Down & myeloid associated differentiation marker \\
\hline FBRS & -0.76411 & $1.24 \mathrm{E}-05$ & 0.001918 & -4.36972 & Down & fibrosin \\
\hline MRPL49 & -0.7639 & 0.002087 & 0.040327 & -3.07756 & Down & mitochondrial ribosomal protein L49 \\
\hline VSIR & -0.76379 & 0.00045 & 0.017213 & -3.50884 & Down & V-set immunoregulatory receptor \\
\hline RIC8A & -0.76185 & 0.00077 & 0.023545 & -3.36328 & Down & RIC8 guanine nucleotide exchange factor A \\
\hline NCOR2 & -0.75973 & 0.000223 & 0.011379 & -3.6918 & Down & nuclear receptor corepressor 2 \\
\hline RARA & -0.75606 & 0.000262 & 0.012485 & -3.64997 & Down & retinoic acid receptor alpha \\
\hline DPYD & -0.75537 & 5.07E-05 & 0.004486 & -4.05246 & Down & dihydropyrimidine dehydrogenase \\
\hline
\end{tabular}




\begin{tabular}{|c|c|c|c|c|c|c|}
\hline DMXL1 & -0.75215 & 5.33E-06 & 0.001228 & -4.55131 & Down & Dmx like 1 \\
\hline PNISR & -0.75092 & 0.000348 & 0.014718 & -3.57662 & Down & PNN interacting serine and arginine rich protein \\
\hline DGKD & -0.75064 & $6.00 \mathrm{E}-05$ & 0.004932 & -4.013 & Down & diacylglycerol kinase delta \\
\hline USP22 & -0.75061 & 2.41E-08 & 4.04E-05 & -5.57939 & Down & ubiquitin specific peptidase 22 \\
\hline WDFY2 & -0.74983 & 0.002146 & 0.0409 & -3.06921 & Down & WD repeat and FYVE domain containing 2 \\
\hline TBC1D10C & -0.7491 & 0.002373 & 0.042779 & -3.03907 & Down & TBC1 domain family member $10 \mathrm{C}$ \\
\hline SH3BGRL3 & -0.7484 & 0.001537 & 0.033863 & -3.16754 & Down & SH3 domain binding glutamate rich protein like 3 \\
\hline CTDNEP1 & -0.74753 & 0.000679 & 0.022149 & -3.39777 & Down & CTD nuclear envelope phosphatase 1 \\
\hline FAXDC2 & -0.74702 & 0.000422 & 0.016539 & -3.52616 & Down & fatty acid hydroxylase domain containing 2 \\
\hline B4GALT1 & -0.74485 & 0.000115 & 0.007342 & -3.85717 & Down & beta-1,4-galactosyltransferase 1 \\
\hline PLCB2 & -0.74418 & 0.001646 & 0.035404 & -3.14758 & Down & phospholipase $\mathrm{C}$ beta 2 \\
\hline MIDEAS & -0.74414 & 3.63E-05 & 0.00378 & -4.12956 & Down & mitotic deacetylase associated SANT domain protein \\
\hline AKNA & -0.74392 & 0.000222 & 0.011379 & -3.693 & Down & AT-hook transcription factor \\
\hline CNDP2 & -0.74239 & 0.000741 & 0.023226 & -3.374 & Down & carnosine dipeptidase 2 \\
\hline MDM4 & -0.7411 & 7.01E-06 & 0.001447 & -4.4934 & Down & MDM4 regulator of $\mathrm{p} 53$ \\
\hline LGALSL & -0.73919 & 0.002938 & 0.047889 & -2.97417 & Down & galectin like \\
\hline MAN2B1 & -0.73902 & 0.001842 & 0.03779 & -3.11459 & Down & mannosidase alpha class $2 \mathrm{~B}$ member 1 \\
\hline ATP2A2 & -0.7385 & 0.000899 & 0.025541 & -3.32034 & Down & ATPase sarcoplasmic/endoplasmic reticulum $\mathrm{Ca} 2+$ transporting 2 \\
\hline DRAP1 & -0.73622 & 0.002476 & 0.043726 & -3.02631 & Down & DR1 associated protein 1 \\
\hline MGLL & -0.73517 & 0.000524 & 0.018855 & -3.46803 & Down & $\begin{array}{l}\text { monoglyceride lipase } \\
\text { carbamoyl-phosphate synthetase 2, aspartate transcarbamylase, and }\end{array}$ \\
\hline $\mathrm{CAD}$ & -0.73466 & 0.003059 & 0.048926 & -2.96174 & Down & dihydroorotase \\
\hline CASP2 & -0.73357 & 3.92E-05 & 0.003894 & -4.11195 & Down & caspase 2 \\
\hline ZMIZ1 & -0.72916 & $1.07 \mathrm{E}-05$ & 0.001778 & -4.40181 & Down & zinc finger MIZ-type containing 1 \\
\hline RIN3 & -0.72851 & $4.25 \mathrm{E}-06$ & 0.001071 & -4.5987 & Down & Ras and Rab interactor 3 \\
\hline USP39 & -0.72837 & 0.000328 & 0.0142 & -3.59223 & Down & ubiquitin specific peptidase 39 \\
\hline STK10 & -0.72431 & $2.47 \mathrm{E}-05$ & 0.003077 & -4.21788 & Down & serine/threonine kinase 10 \\
\hline GUCY1A1 & -0.724 & 0.003054 & 0.048926 & -2.96223 & Down & guanylate cyclase 1 soluble subunit alpha 1 \\
\hline YLPM1 & -0.72338 & 0.000607 & 0.020764 & -3.42834 & Down & YLP motif containing 1 \\
\hline ARHGAP4 & -0.72319 & 0.000404 & 0.016147 & -3.53719 & Down & Rho GTPase activating protein 4 \\
\hline LEF1 & -0.72136 & 0.00139 & 0.032205 & -3.19676 & Down & lymphoid enhancer binding factor 1 \\
\hline CMTM6 & -0.72086 & 0.002409 & 0.043137 & -3.03453 & Down & CKLF like MARVEL transmembrane domain containing 6 \\
\hline $\mathrm{E} 2 \mathrm{~F} 3$ & -0.71983 & 0.001503 & 0.033554 & -3.17415 & Down & E2F transcription factor 3 \\
\hline WIPF1 & -0.71966 & $5.60 \mathrm{E}-07$ & 0.000259 & -5.00459 & Down & WAS/WASL interacting protein family member 1 \\
\hline CALM3 & -0.71921 & 0.000348 & 0.014718 & -3.57652 & Down & calmodulin 3 \\
\hline RAB8A & -0.71773 & 0.001282 & 0.030728 & -3.21993 & Down & RAB8A, member RAS oncogene family \\
\hline PLEK & -0.71655 & $4.74 \mathrm{E}-07$ & 0.000224 & -5.03635 & Down & pleckstrin \\
\hline SEPTIN9 & -0.71354 & $3.22 \mathrm{E}-06$ & 0.000876 & -4.65642 & Down & septin 9 \\
\hline RN7SK & -0.71244 & 0.002277 & 0.042026 & -3.05157 & Down & RNA component of 7SK nuclear ribonucleoprotein \\
\hline IRAK3 & -0.71152 & 7.53E-06 & 0.001488 & -4.47822 & Down & interleukin 1 receptor associated kinase 3 \\
\hline DOCK2 & -0.70951 & $1.09 \mathrm{E}-05$ & 0.001787 & -4.39763 & Down & dedicator of cytokinesis 2 \\
\hline SRRM2 & -0.70753 & $8.83 \mathrm{E}-07$ & 0.000367 & -4.91617 & Down & serine/arginine repetitive matrix 2 \\
\hline M6PR & -0.70709 & 0.00093 & 0.026107 & -3.31085 & Down & mannose-6-phosphate receptor, cation dependent \\
\hline
\end{tabular}




\begin{tabular}{ccccccc}
\hline SCAF4 & -0.70556 & 0.001328 & 0.031434 & -3.20978 & Down & SR-related CTD associated factor 4 \\
MYO9B & -0.70522 & 0.000538 & 0.019202 & -3.46104 & Down & myosin IXB \\
DENND4C & -0.7041 & $6.85 \mathrm{E}-05$ & 0.005343 & -3.98142 & Down & DENN domain containing 4C \\
KRAS & -0.70354 & $1.53 \mathrm{E}-05$ & 0.002227 & -4.32488 & Down & KRAS proto-oncogene, GTPase \\
PTPN18 & -0.70306 & $5.90 \mathrm{E}-05$ & 0.004918 & -4.01685 & Down & protein tyrosine phosphatase non-receptor type 18 \\
ATE1 & -0.70243 & 0.00107 & 0.02782 & -3.27142 & Down & arginyltransferase 1 \\
PREX1 & -0.7024 & 0.000846 & 0.024636 & -3.33725 & Down & phosphatidylinositol-3,4,5-trisphosphate dependent Rac exchange factor 1 \\
SLC44A2 & -0.70141 & 0.001156 & 0.029251 & -3.24948 & Down & solute carrier family 44 member 2 \\
CIC & -0.70058 & 0.002324 & 0.042463 & -3.04542 & Down & capicua transcriptional repressor \\
HDAC7 & -0.6961 & 0.001403 & 0.032317 & -3.19412 & Down & protein kinase N1 \\
PKN1 & -0.6958 & $2.53 \mathrm{E}-05$ & 0.003077 & -4.21223 & Down & solute carrier family 6 member 6 \\
SLC6A6 & -0.69526 & 0.000535 & 0.019133 & -3.46241 & Down & actin beta \\
ACTB & -0.69456 & 0.000231 & 0.011467 & -3.68263 & Down & WRN helicase interacting protein 1 \\
WRNIP1 & -0.69294 & 0.002658 & 0.045308 & -3.0048 & Down & glucose-6-phosphate dehydrogenase \\
G6PD & -0.69232 & $3.13 \mathrm{E}-05$ & 0.003441 & -4.16381 & Down &
\end{tabular}

Table 2 The enriched GO terms of the up and down regulated differentially expressed genes

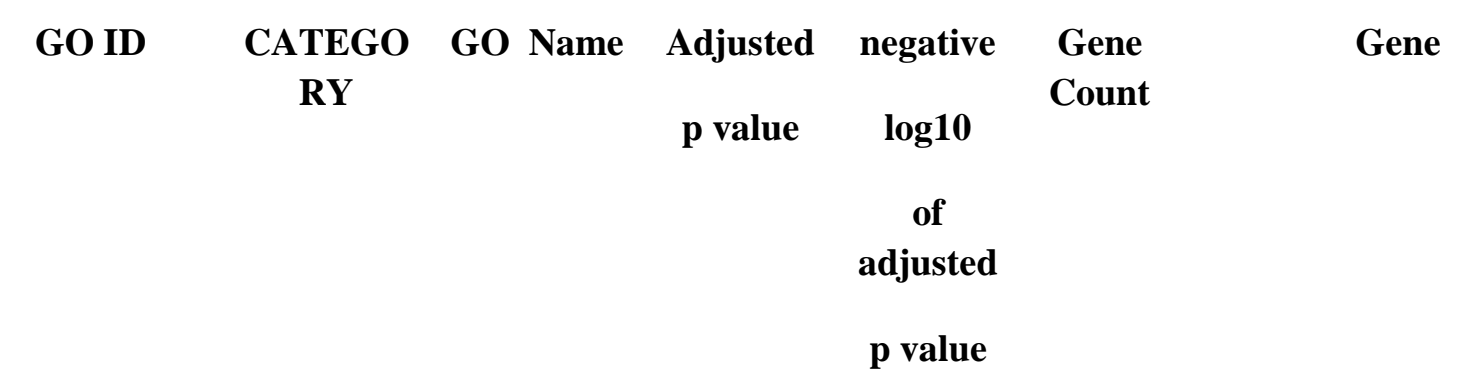

\begin{tabular}{|c|c|c|c|c|c|c|}
\hline \multicolumn{7}{|c|}{ Up regulated genes } \\
\hline GO:0032501 & $\mathrm{BP}$ & $\begin{array}{l}\text { multicellul } \\
\text { ar } \\
\text { organismal } \\
\text { process }\end{array}$ & $3.03 \mathrm{E}-09$ & $\begin{array}{c}8.517864 \\
45\end{array}$ & 199 & $\begin{array}{c}\text { OPN1LW,NDP,HTN3,A } \\
\text { DAM18,SERPINA10,SP } \\
\text { ATA22,LGR5,OCA2,CD } \\
\text { H3,PAEP,SIX1,SLC34A } \\
\text { 2,DSG4,ASPA,SPATA16 } \\
\text {,GREM1,LRCOL1,TXN } \\
\text { DC8,GLRA3,NEUROD4, } \\
\text { MEI4,ESRP1,KRT85,EP } \\
\text { HA3,PTN,CNGA1,CHR } \\
\text { NB3,GHRHR,SHCBP1L, } \\
\text { MYH4,MEIOC,GPR37L } \\
\text { 1,CYP26A1,CYP2J2,WD } \\
\text { R72,BNC1,AQP4,BMPR } \\
\text { 1B,IMPG1,MYH8,KRT1 } \\
\text { 4,SLITRK6,VGLL2,RB } \\
\text { M46,ROS1,EYA4,KCNH }\end{array}$ \\
\hline
\end{tabular}


6,SCN7A,GABRG1,SFT

PC,RIMS2,SCEL,MYO3

A,NLGN4X,LBP,DMRT

A1,MYF6,RP1,TEKT3,F

EZF1,SGCD,BRINP1,V

XN,GRHL2,SLC26A5,E

VC,POU3F3,WNT2,LVR

N,CBLN1,CLDN12,STM

ND1,LRFN5,GLP1R,SC

N11A,GABRP,SLC16A1

2,DRP2,GCM2,MC1R,S

LC1A1,CXCL14,FLG,R

GN,TRIM54,ID4,NPY2R

,SERPINB13,TRPM1,NE

UROG1,F9,ROBO2,GPC

6,WNT9A,GREM2,KCN

D2,PHLDA2,DACH2,PL

A2G2D,MFAP5,GABRB

1,AZGP1,SLC4A10,PTP

N3,HAS2,CTNNA2,IGS

F3,IGSF21,PCDHGA9,K

RT24,ANHX,KIT,IHO1,

ZNF750,CNTN5,CNTN4

,CFAP53,NKAPL,WNT5

A,ADGRF1,EDA,KCNA

1,TRIM71,TDRD6,SCN1

A,MROH2B,TBX18,HS

D11B2,SCN2A,TACC2,

KCNK1,ADGRV1,PTHL

H,CCNA1,COBL,ACVR

1C,FAP,CDH8,HUNK,G

DA,FERMT1,PCDHAC2

,DACT3,THSD7A,KRT8

0,DGKB,IRX2,ABCA4,S

TRC,PTH2R,POPDC2,A

DCY2,NEUROD1,SPIN

K5,KCNIP3,EFHD1,IL20

RA,CLDN1,MAL2,IZU

MO1,SHROOM3,SOHL

H1,DISP3,GABRB3,ETV

1,CYP24A1,TNR,ERRFI

1,PKHD1,KRT13,SLIT2,

MAST1,DTNA,ANTXR1

,ITGA8,GJC1,WNK3,PP 


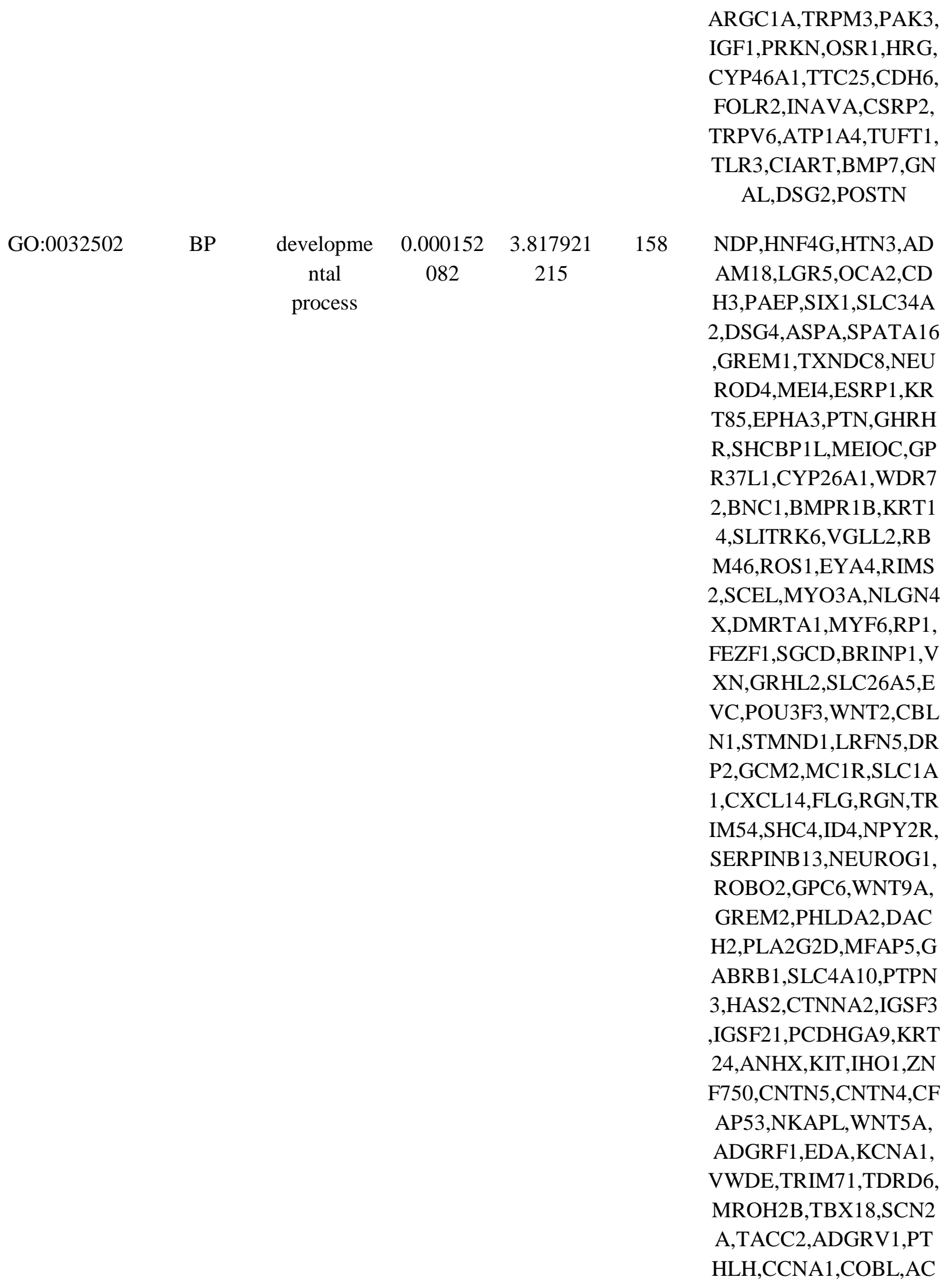




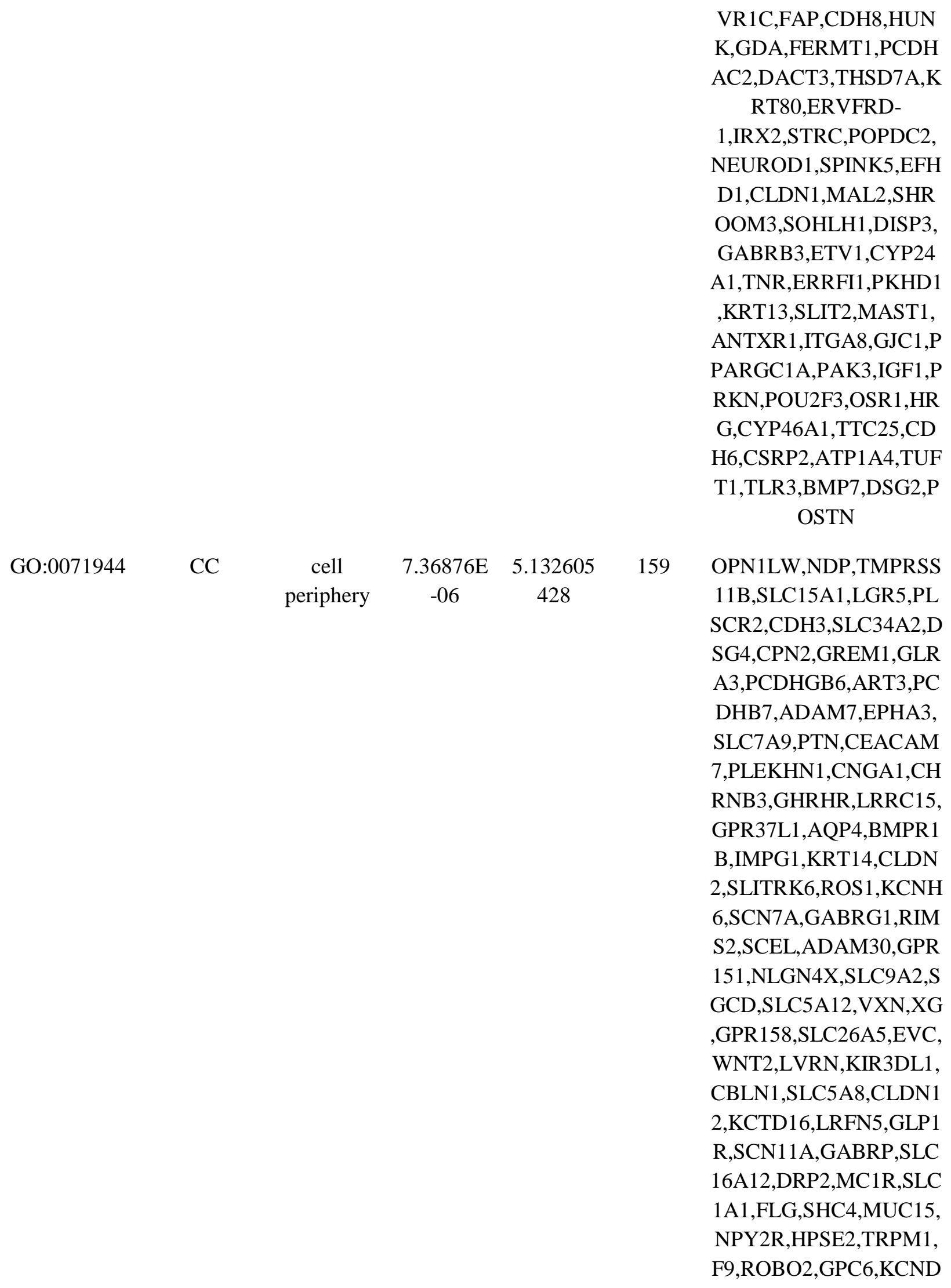




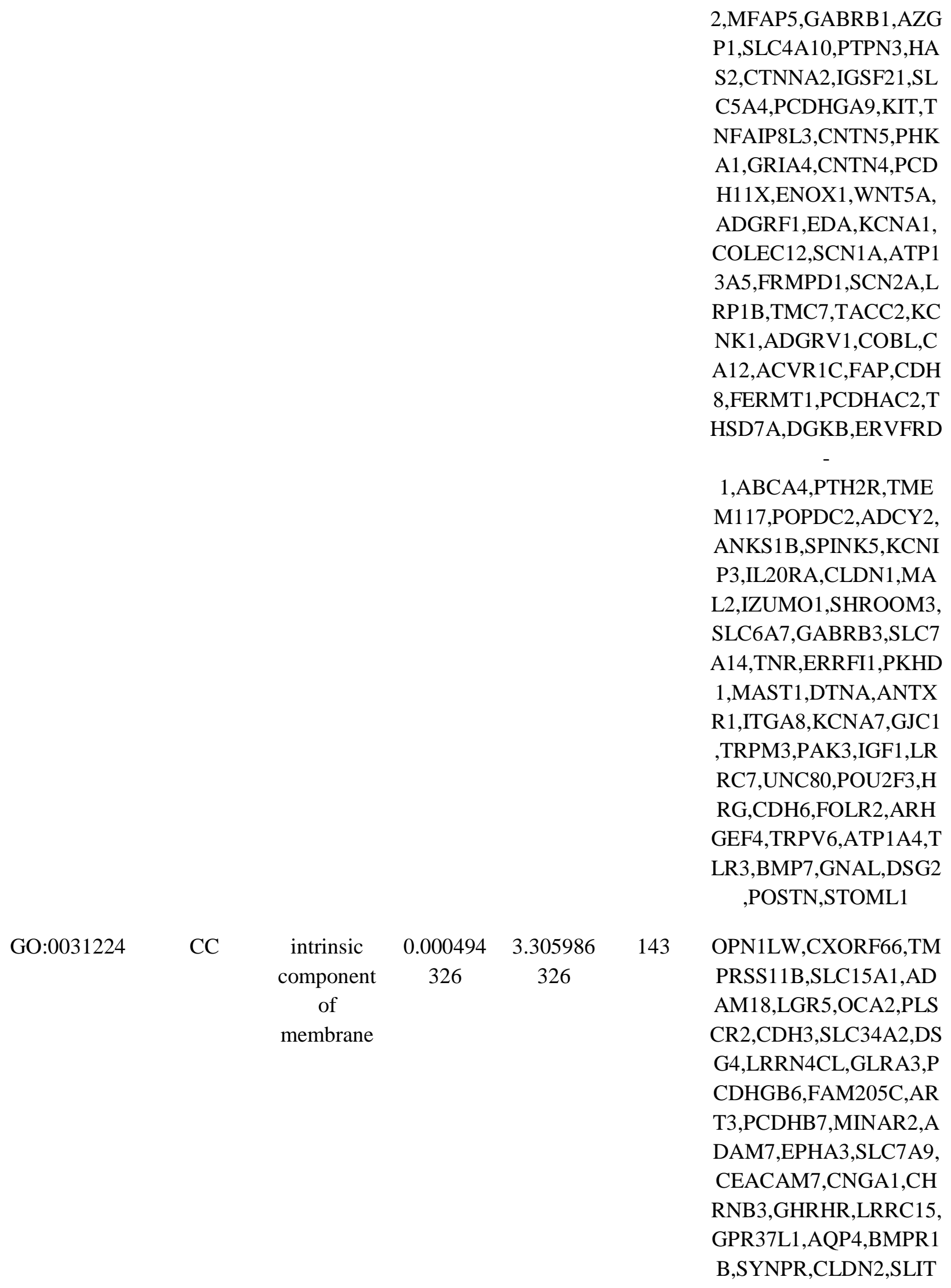




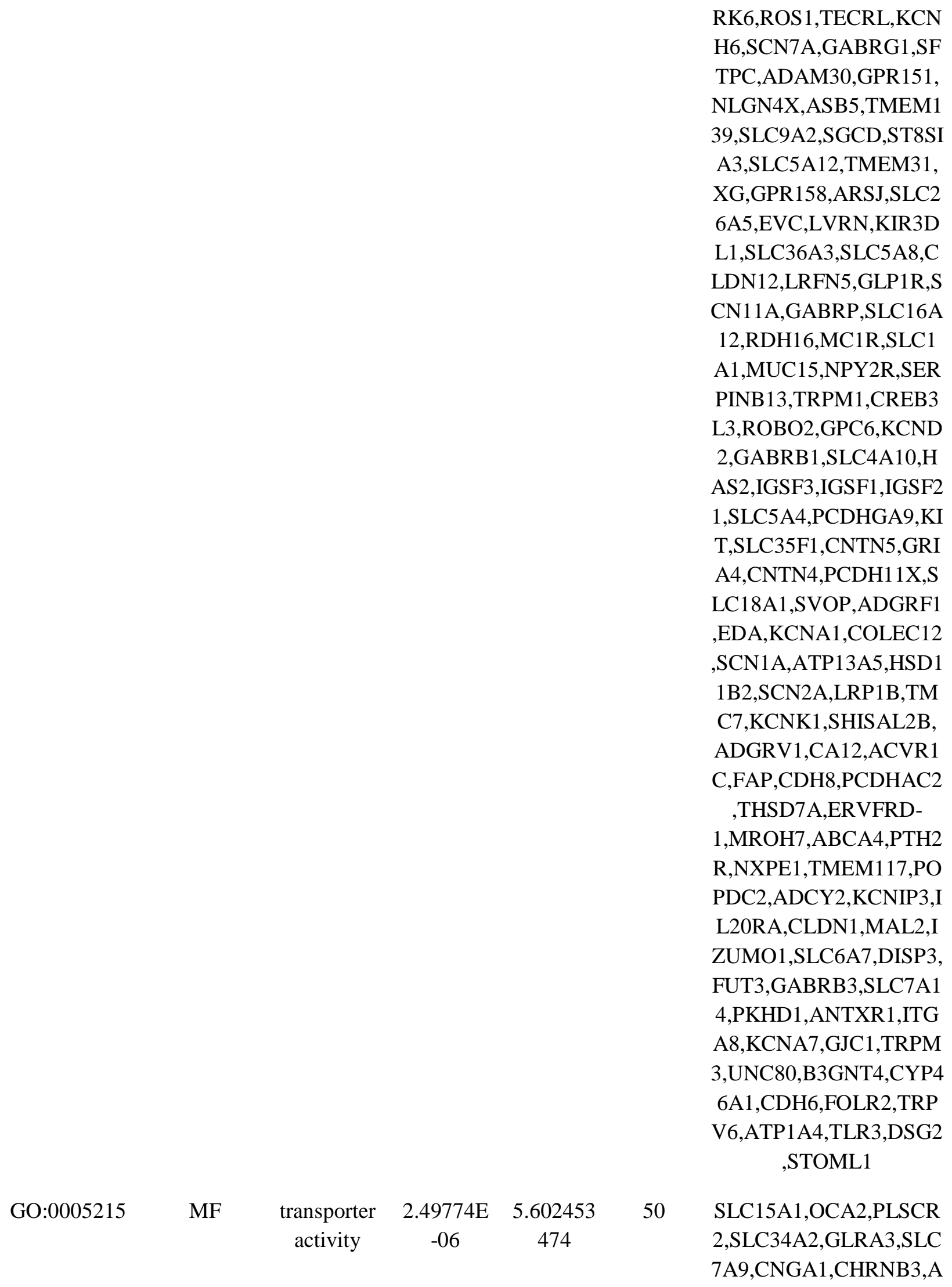




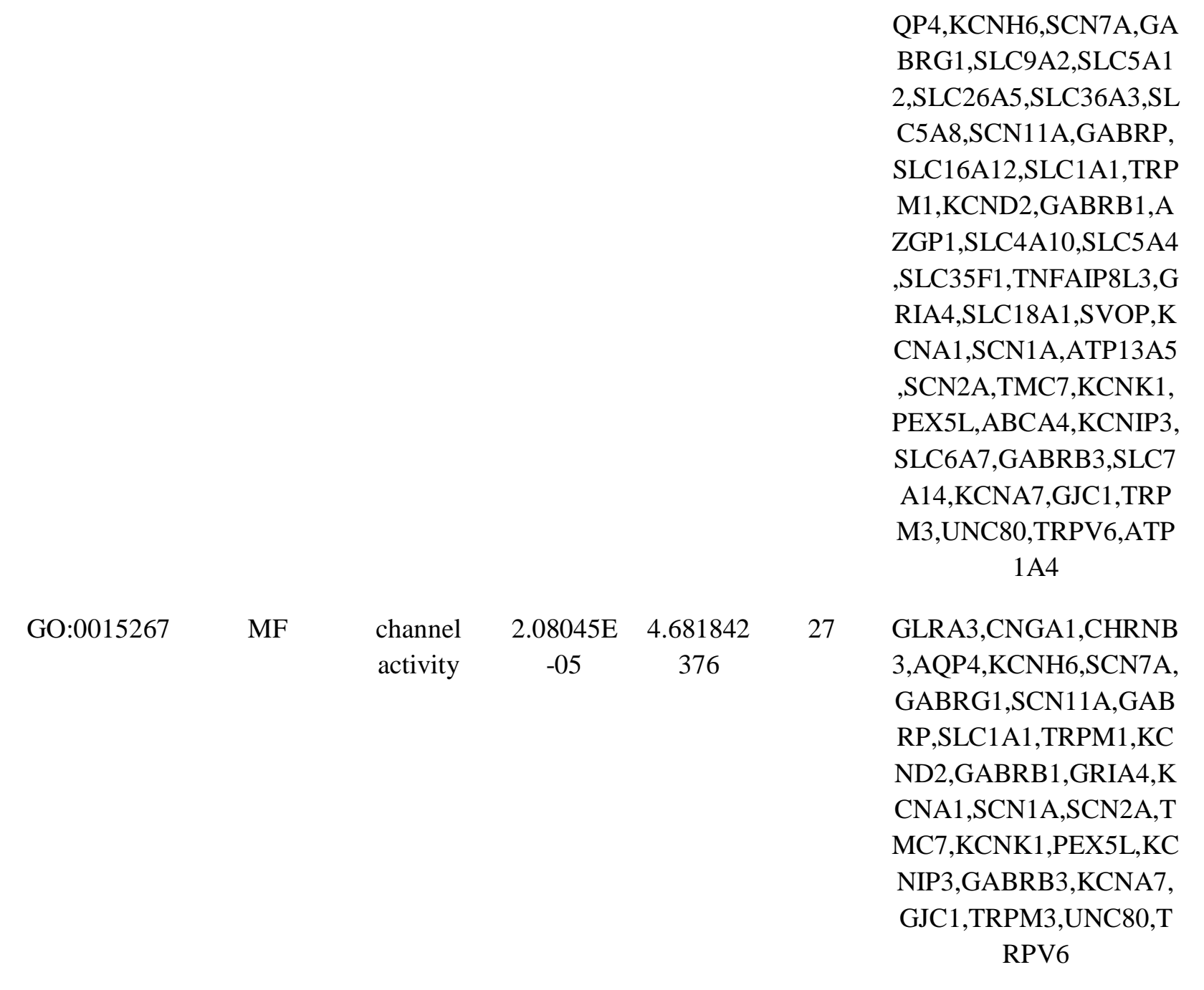

Down regulated genes

\begin{tabular}{|c|c|c|c|c|c|c|}
\hline GO:0001775 & $\mathrm{BP}$ & $\begin{array}{c}\text { cell } \\
\text { activation }\end{array}$ & $4.37 \mathrm{E}-37$ & $\begin{array}{c}36.36000 \\
553\end{array}$ & 125 & $\begin{array}{r}\text { ITGA2B,ITGB3,MPL,IT } \\
\text { GAX,MPIG6B,THBS1,F } \\
\text { LNA,TRPC6,SELP,CD79 } \\
\text { A,HLA- } \\
\text { A,VCL,PYGB,TLN1,P2 } \\
\text { RY1,FCRL1,GP5,TREM } \\
\text { L1,NOTCH2,F2RL3,ML } \\
\text { EC,PSAP,ACAA1,PTPRJ } \\
\text {,LRP1,BAX,P2RX1,CLU } \\
\text {,FCN1,CDKN1A,NCSTN } \\
\text {,SMAD3,GLIPR1,LFNG, } \\
\text { CTSA,ITGB1,ANO6,CA } \\
\text { PN1,BAK1,IGHM,MS4A } \\
\text { 1,FERMT3,TLR2,RNAS } \\
\text { ET2,GPR183,TCIRG1,H }\end{array}$ \\
\hline
\end{tabular}




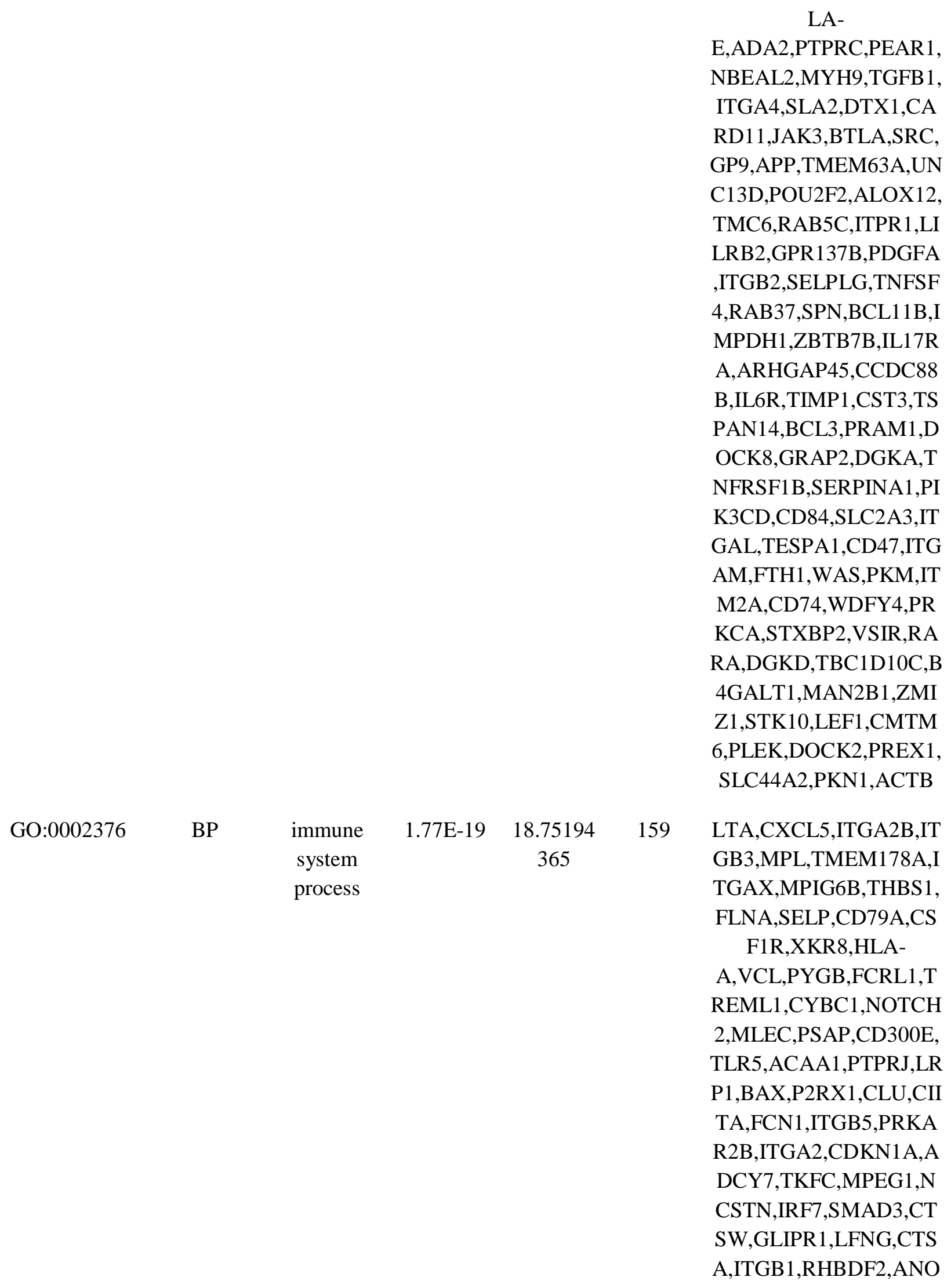




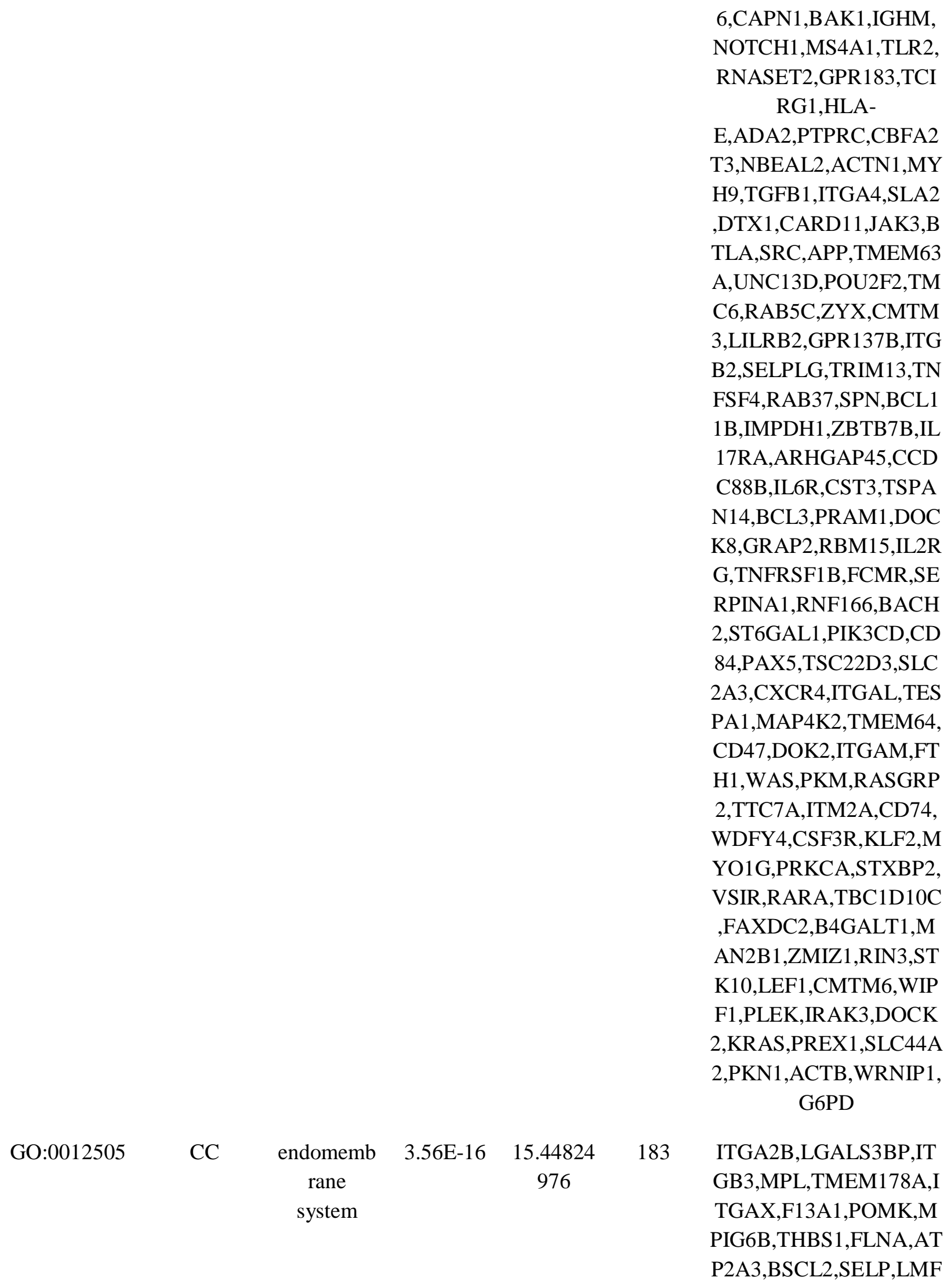


2,CD79A,RAB27B,NUP 210,HLA-

A,VCL,PYGB,VCAN,TR EML1,CYBC1,NOTCH2, MLEC,PSAP,CHST12,A CAA1,ABCA7,PTPRJ,L RP1,BAX,SUN2,P2RX1, SPARC,CLU,TBXA2R,F CN1,PPM1L,PANX1,NR GN,RAB1B,NCSTN,IRF 7,CHERP,SMAD3,CTS W,GLIPR1,LFNG,TME M259,CTSA,ITGB1,TCL 1A,RHBDF2,DENND4B, ANO6,VPS52,CAPN1,B AK1,NOTCH1,SRPRA,F ERMT3,TLR2,RNASET 2,TCIRG1,ESYT1,HLAE,TPP1,MMD,ADA2,LE PROT,LHFPL2,PTPRC, CBFA2T3,SH3BGRL2,N BEAL2,ACTN1, GALNT 10,MYH9,CHPF2,TGFB 1,SLC35E2B,SLA2,PITP NM2,EHD3,JAK3,ITM2

B,SRC,DSE,APP,PCSK6, FGD3,TMEM63A,UNC1 3D,TGOLN2,TMC6,STI M1,RAB5C,CMTM3,CN ST,ITPR1,GANAB,LDL RAP1,LILRB2,EPM2AIP 1,SELENON,RUFY1,PD GFA,ITGB2,ATP9A,TRI M13,VPS37B,GDI1,RAB 37,IMPDH1,ARHGAP45 ,CCDC88B,FGD2,TIMP1 ,CST3,TSPAN14,STON2 ,GRAP2,RBM15,ARL6IP 5,LRBA,IL2RG,PACSIN 2,MGAT4B,TNFRSF1B, SERPINA1,CYTH4,MIN K1,ST6GAL1,PIGS,SLC 2A3,RNF167,CXCR4,IT GAL,TESPA1,MAP4K2, 


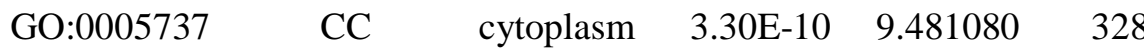

TNRC18,TMEM64,CTT

N,CD47,NUP188,APLP2

,ITGAM,DAD1,FTH1,P KM,CHST15,TNIK,ITM

2A,CD74,TMEM159,WD

FY4,CHMP1A,VPS51,P RKCA,STXBP2,DGKD, WDFY2,TBC1D10C,CT DNEP1,FAXDC2,B4GA LT1,MAN2B1,ATP2A2, MGLL,RIN3,STK10,AR HGAP4,CMTM6,CALM 3,RAB8A,DOCK2,M6PR ,DENND4C,KRAS,SLC4 4A2,PKN1

96

COX4I2,ITGA2B,TMEM 70,TMEM150A,LGALS3 BP,ITGB3,CCDC28B,SP SB2,MPL,EXO5,TMEM 178A,ITGAX,F13A1,PO MK,MPIG6B,THBS1,FL NA,ACAD10,TUBB1,AT P2A3,BSCL2,ARHGAP2 7,TRPC6,SELP,LMF2,C D79A,MEMO1,RAB27B, MAP3K7CL,NUP210,HL A-

A,VCL,PYGB,ZNF185,T LN1,ATG16L2,P2RY1,V CAN,TREML1,ENDOD1 ,CYBC1,NOTCH2,MLE C,LIPA,PSAP,CHST12,L IMS1,MYBBP1A,ACAA 1,ABCA7,ELAC2,CAVI N2,PTPRJ,LRP1,BAX,S UN2,P2RX1,DENND1C, PYCR2,SMCR8,ENO2,S PARC,CLU,TBXA2R,LR RC25,CIITA,FCN1,ITGB 5,PRKAR2B,ITGA2,PP M1L,CDKN1A,TKFC,Z C3HAV1L,MPEG1,PAN X1,NRGN,RAB1B,PDE5 
A,CLPP,NCSTN,IRF7,C HERP,SMAD3,CTSW,G LIPR1,CMIP,LFNG,TM EM259,MFAP3L,CTSA,I TGB1,TCL1A,RHBDF2, MRPL28,DENND4B,AN O6,SIDT2,VPS52,CAPN 1,BAK1,NOTCH1,PLEC, SRPRA,FERMT3,TLR2, RNASET2,DNAH1,TCI

RG1,ESYT1,HLAE,TPP1,C1ORF198,MM D,ARHGAP6,INF2,ADA 2,LEPROT,LHFPL2,PTP RC,SH3BP1,CBFA2T3,N BEAL2,USP20,ACTN1, GALNT10,MYH9,EML3 ,CHPF2,OGT,TGFB1,SL C35E2B,SLA2,PITPNM2 ,DTX1,CARD11,MADD, MRTFA,EHD3,JAK3,SM G5,ITM2B,SRC,DSE,AP P,PCSK6,FGD3,TMEM6 3A,UNC13D,POU2F2,T GOLN2,ALOX12,FHOD 1,TMC6,MAP1A,STIM1, FTSJ1,RAB5C,ZYX,GU CY1B1,CMTM3,CNST,I TPR1,GANAB,LDLRAP 1,RNF213,MYLK,CENP T,LILRB2,TYMP,EPM2 AIP1,SELENON,RUFY1 ,GNB5,PRUNE1,HELZ2, GPR137B,MTPN,PDGF A,ITGB2,ATP9A,DAPP1 ,CORO1B,TRIM13,SIN3 B,VPS37B,UPF1,GDI1,P GAP6,DIMT1,RAB37,I MPDH1,UBA7,ARHGA P45,IL10RA,CCDC88B, FGD2,TIMP1,CST3,AR HGEF18,FPGT,TSPAN1 4,BCL3,CASP6,TUBA4 A,STON2,GAS2L1,DOC 
K8,GRAP2,C15ORF39,D

GKA,ARHGEF1,EVL,A

RL6IP5,LRBA,IL2RG,P

ACSIN2,MGAT4B,TNF

RSF1B,LIMD2,MOB1B, SERPINA1,PARVB,CYT H4,MINK1,CDK9,RNF1

66,BACH2,ST6GAL1,PI K3CD,PIGS,PAX5,TSC2 2D3,PI4KA,SLC2A3,CD K2AP1,RNF167,CXCR4, ITGAL,TESPA1,ZNF641 ,AFF3,MAP4K2,TNRC1 8,TMEM64,CTTN,CD47, ABI3,NUP188,DOK2,AP LP2,RNH1,ITGAM,DAD 1,FTH1,WAS,PKM,MIC AL1,RASGRP2,CHST15, TTC7A,NKTR,TNIK,IT M2A,CD74,TMEM159, WDFY4,MINDY1,STRN 4,ARHGAP18,MTHFR, MYO1G,MBNL1,CHMP 1A,VPS51,PRKCA,FOX K1,STXBP2,MYADM,M RPL49,RIC8A,RARA,DP YD,DMXL1,PNISR,DG KD,WDFY2,TBC1D10C, SH3BGRL3,CTDNEP1,F AXDC2,B4GALT1,PLC B2,AKNA,CNDP2,MAN 2B1,ATP2A2,MGLL,CA D,CASP2,ZMIZ1,RIN3,S TK10,GUCY1A1,YLPM 1,ARHGAP4,LEF1,CMT M6,WIPF1,CALM3,RAB 8A,PLEK,SEPTIN9,IRA K3,DOCK2,M6PR,MYO 9B,DENND4C,KRAS,PT PN18,ATE1,PREX1,SLC 44A2,HDAC7,PKN1,AC TB,WRNIP1,G6PD

GO:0019899 MF $\quad$ enzyme 2.39E-07 6.621960 90 ITGB3,UBOX5,ITGAX,


binding

GO:0005515

\begin{abstract}
MF
\end{abstract}
protein

binding
$0.000108 \quad 3.966126$ 112 242

FLNA,TRPC6,CSF1R,V CL,NOTCH2,MLEC,PS AP,LIMS1,CAVIN2,PTP RJ,LRP1,SMCR8,CLU,N FRKB,PRKAR2B,CDKN 1A,PANX1,CLPP,NCST N,SMAD3,ITGB1,TCL1 A,PIK3IP1,NOTCH1,TCI RG1,ARHGAP6,INF2,PT PRC,NBEAL2,PITPNM2 ,DTX1,JAK3,SMG5,SRC ,APP,FGD3,UNC13D,ST IM1,CNST,SCAF1,LILR B2,ITGB2,ATP9A,GDI1, ZBTB7B,FGD2,IL6R,TI MP1,CST3,TSPAN14,TU BA4A,PRAM1,LRBA,T NFRSF1B,MOB1B,SER PINA1,CDK9,SLC12A6, CDK2AP1,CXCR4,MAP 4K2,DOK2,WAS,MICA L1,CD74,STRN4,PRKC A,VSIR,NCOR2,RARA, DGKD,USP22,MDM4,A TP2A2,CAD,CASP2,RIN 3,LEF1,CALM3,RAB8A, PLEK,SCAF4,MYO9B,P REX1,HDAC7,PKN1,AC TB

\section{0}

LTA,CCDC24,CXCL5,IT GA2B,TMEM70,TMEM 150A,LGALS3BP,ITGB3 ,CCDC28B,CNNM4,SPS B2,SEC14L5,PLXNB2,M PL,UBOX5,ITGAX,F13 A1,POMK,MPIG6B,THB S1,FLNA,ATP2A3,BSCL 2,ARHGAP27,TRPC6,SE LP,LMF2,CD79A,CSF1R ,MEMO1,RAB27B,MAP 3K7CL,ZNF296,HLAA,VCL,PYGB,ZNF185,T LN1,ATG16L2,P2RY1,V 
CAN,GP5,TREML1,EN

DOD1,SF3A2,CYBC1,N

OTCH2,MLEC,PSAP,FA

M185A,CD300E,LIMS1, MYBBP1A,TLR5,ACAA

1,CAVIN2,PTPRJ,MYC

L,LRP1,BAX,SUN2,P2R

X1,DENND1C,PYCR2,S

MCR8,ENO2,SPARC,T

MEM140,CLU,TBXA2R, LRRC25,CIITA,FCN1,N

FRKB,ITGB5,PRKAR2B

,ITGA2,CDKN1A,CCNL

1,MAP4K1,TKFC,PANX

1,NRGN,RAB1B,PDE5A

,CLPP,NCSTN,IRF7,CH

ERP,SMAD3,GLIPR1,NI

BAN3,C2ORF88,CMIP, MFAP3L,KDM2B,CMT

M5,ITGB1,TCL1A,RHB

DF2,MRPL28,DENND4

B,PIK3IP1,ANO6,VPS52

,CAPN1,BAK1,IGHM,P

OLR1A,NOTCH1,MS4A

1,PLEC,FERMT3,TLR2, GSE1,DNAH1,TCIRG1,

PLXDC2,ESYT1,GIGYF

$$
\text { 1,HLA- }
$$

E,TPP1,LENG8,MMD,A RHGAP6,INF2,ADA2,L EPROT,LHFPL2,RSRP1, PTPRC,PEAR1,SH3BP1, CBFA2T3,SH3BGRL2,N BEAL2,USP20,ACTN1, GALNT10,MYH9,EML3 ,OGT,TGFB1,ITGA4,SL A2,PITPNM2,DTX1,CA RD11,MADD,KCNA3,M RTFA,EHD3,JAK3,SMG 5,BTLA,ITM2B,SRC,GP 9,APP,PCSK6,FGD3,TM EM63A,UNC13D,POU2 F2,TGOLN2,ALOX12,F HOD1,TMC6,MAP1A,S 
TIM1,CTDSPL,NHSL2,F TSJ1,RAB5C,ZYX,GUC Y1B1,CMTM3,CNST,IT PR1,GANAB,LDLRAP1, CCNL2,SCAF1,MYLK,C ENPT,ARL15,SRSF6,LI LRB2,TYMP,EPM2AIP1 ,SELENON,SNRPA,RUF Y1,GNB5,PRUNE1,HEL Z2,GPR137B,MBD6,MT PN,PDGFA,ITGB2,SELP LG,ATP9A,DAPP1,COR O1B,TRIM13,SIN3B,VP S37B,UPF1,GDI, TNFS F4,PGAP6,RPA1,RAB37 ,SPN,BCL11B,IMPDH1, ZBTB7B,UBA7,IL17RA, ARHGAP45,CBX6,FAM 78A,IL10RA,CCDC88B, FGD2,IL6R,TIMP1,CST 3,ARHGEF18,SUSD3,TS PAN14,BCL3,CASP6,TU BA4A,STON2,GAS2L1, PRAM1,DOCK8,FAM11 7B,GRAP2,ARHGEF1,R BM15,EVL,ARL6IP5,LR BA,IL2RG,PACSIN2,M GAT4B,TNFRSF1B,LIM D2,MOB1B,MEPCE,SE RPINA1,PARVB,CYTH 4,MINK1,CDK9,RNF166 ,BACH2,C16ORF54,ST6 GAL1,PIK3CD,CD84,PI GS,PAX5,SLC12A6,TSC 22D3,PI4KA,SLC2A3,C DK2AP1,RNF167,CXCR 4,ITGAL,TESPA1,MAP4 K2,CTTN,CD47,ABI3,D OK2,JADE2,APLP2,PRR C2B,RNH1,ITGAM,FTH 1,WAS,PKM,MICAL1,M ED12,CHST15,TTC7A,R NF44,TNIK,ACTR8,ITM 2A,CD74,TMEM159,WD 
FY4,MINDY1,SETD1B, STRN4,ARHGAP18,CSF 3R,KLF2,MYO1G,MBN L1,CHMP1A,TSC22D4, VPS51,PRKCA,FOXK1, STXBP2,MYADM,MRP L49,VSIR,RIC8A,NCOR 2,RARA,DPYD,DMXL1, DGKD,USP22,WDFY2,T BC1D10C,SH3BGRL3,C TDNEP1,FAXDC2,B4G ALT1,PLCB2,AKNA,M DM4,LGALSL,ATP2A2, DRAP1,MGLL,CAD,CA SP2,ZMIZ1,RIN3,USP39 ,STK10,GUCY1A1,YLP M1,ARHGAP4,LEF1,C MTM6,E2F3,WIPF1,CA LM3,RAB8A,PLEK,SEP TIN9,IRAK3,DOCK2,SR RM2,M6PR,SCAF4,MY O9B,DENND4C,KRAS,P TPN18,ATE1,PREX1,CI C,HDAC7,PKN1,ACTB, WRNIP1,G6PD

Table 3 The enriched pathway terms of the up and down regulated differentially expressed genes

\begin{tabular}{cccc} 
Pathway ID Pathway Name & $\begin{array}{c}\text { Adjusted } \\
\text { p value }\end{array}$ & $\begin{array}{c}\text { Negative } \\
\log 10 \text { of } \\
\text { adjusted } \\
\text { p value }\end{array}$ & Gene \\
& \multicolumn{4}{c}{$c$}
\end{tabular}

\begin{tabular}{llllll}
\hline \multicolumn{5}{c}{ Up regulated genes } \\
\hline $\begin{array}{l}\text { REAC:R-HSA- } \\
6809371\end{array}$ & $\begin{array}{l}\text { Formation of the } \\
\text { cornified envelope }\end{array}$ & 0.017403 & 1.759373 & 10 & LIPJ,DSG4,KRT85,KRT \\
& 093 & 564 & & $\begin{array}{l}\text { 14,FLG,KRT24,KRT80,S } \\
\text { PINK5,KRT13,DSG2 }\end{array}$ \\
REAC:R-HSA- & & & & Cell-cell junction \\
421270 & 0.057968 & 1.236808 & 6 & CDH3,CLDN2,CLDN12, \\
& organization & 43 & 462 & & CDH8,CLDN1,CDH6
\end{tabular}




\begin{tabular}{|c|c|c|c|c|c|}
\hline $\begin{array}{l}\text { REAC:R-HSA- } \\
373080\end{array}$ & $\begin{array}{l}\text { Class B/2 (Secretin } \\
\text { family receptors) }\end{array}$ & $\begin{array}{l}0.057968 \\
43\end{array}$ & $\begin{array}{l}1.236808 \\
462\end{array}$ & 7 & $\begin{array}{l}\text { GHRHR,WNT2,GLP1R, } \\
\text { WNT9A,WNT5A,PTHL } \\
\text { H,PTH2R }\end{array}$ \\
\hline $\begin{array}{l}\text { REAC:R-HSA- } \\
112316\end{array}$ & Neuronal System & $\begin{array}{l}0.057968 \\
43\end{array}$ & $\begin{array}{l}1.236808 \\
462\end{array}$ & 16 & $\begin{array}{l}\text { GLRA3,CHRNB3,SLITR } \\
\text { K6,KCNH6,NLGN4X,SL } \\
\text { C1A1,KCND2,GABRB1, } \\
\text { GRIA4,KCNA1,KCNK1, } \\
\text { ADCY2,GABRB3,KCN } \\
\text { A7,GJC1,GNAL }\end{array}$ \\
\hline $\begin{array}{l}\text { REAC:R-HSA- } \\
5576891\end{array}$ & Cardiac conduction & $\begin{array}{l}0.058865 \\
891\end{array}$ & $\begin{array}{l}1.230136 \\
275\end{array}$ & 8 & $\begin{array}{l}\text { SCN7A,SCN11A,KCND } \\
\text { 2,SCN1A,SCN2A,KCNK } \\
\text { 1,KCNIP3,ATP1A4 }\end{array}$ \\
\hline $\begin{array}{l}\text { REAC:R-HSA- } \\
382551\end{array}$ & $\begin{array}{l}\text { Transport of small } \\
\text { molecules }\end{array}$ & $\begin{array}{l}0.058865 \\
891\end{array}$ & $\begin{array}{l}1.230136 \\
275\end{array}$ & 23 & $\begin{array}{l}\text { SLC15A1,SLC34A2,SLC } \\
\text { 7A9,AQP4,SLC9A2,SLC } \\
\text { 5A12,SLC5A8,SLC1A1, } \\
\text { TRPM1,CREB3L3,AZG } \\
\text { P1,SLC4A10,SLC5A4, } \\
\text { SLC18A1,ATP13A5,AB } \\
\text { CA4,ADCY2,SLC6A7,W } \\
\text { NK3,TRPM3,UNC80,TR } \\
\text { PV6,ATP1A4 }\end{array}$ \\
\hline
\end{tabular}

Down regulated genes

\begin{tabular}{|c|c|c|c|}
\hline $\begin{array}{ll}\text { REAC:R-HSA- } & \text { Hemostasis } \\
109582 & \end{array}$ & $9.54 \mathrm{E}-20$ & $\begin{array}{ll}19.02047 & 69 \\
251 & \end{array}$ & $\begin{array}{l}\text { ITGA2B,LGALS3BP,IT } \\
\text { GB3,MPL,ITGAX,F13A } \\
\text { 1,MPIG6B,THBS1,FLN } \\
\text { A,TUBB1,ATP2A3,TRP } \\
\text { C6,SELP,RAB27B,VCL, } \\
\text { TLN1,P2RY1,GP5,END } \\
\text { OD1,F2RL3,PSAP,P2RX } \\
\text { 1,SPARC,CLU,TBXA2R } \\
\text {,PRKAR2B,ITGA2,PDE5 } \\
\text { A,CTSW,ITGB1,IGHM, } \\
\text { FERMT3,LHFPL2,ACT } \\
\text { N1,ITGA4,EHD3,SRC,G } \\
\text { P9,APP,STIM1,GUCY1B } \\
\text { 1,ITPR1,GNB5,ITGB2,S } \\
\text { ELPLG,SPN,TIMP1,TU } \\
\text { BA4A,DOCK8,DGKA,S } \\
\text { ERPINA1,CD84,ITGAL, } \\
\text { CD47,DOK2,APLP2,ITG }\end{array}$ \\
\hline
\end{tabular}




\begin{tabular}{|c|c|c|c|c|c|}
\hline $\begin{array}{l}\text { REAC:R-HSA- } \\
6798695\end{array}$ & $\begin{array}{l}\text { Neutrophil } \\
\text { degranulation }\end{array}$ & $6.68 \mathrm{E}-12$ & $\begin{array}{l}11.17530 \\
495\end{array}$ & 46 & $\begin{array}{l}\text { ITGAX,VCL,PYGB,ML } \\
\text { EC,PSAP,ACAA1,PTPRJ } \\
\text {,P2RX1,FCN1,NCSTN,G } \\
\text { LIPR1,CTSA,ANO6,CA } \\
\text { PN1,TLR2,RNASET2,T } \\
\text { CIRG1,ADA2,PTPRC,N } \\
\text { BEAL2,TMEM63A,UNC } \\
\text { 13D,TMC6,RAB5C,LIL } \\
\text { RB2,ITGB2,RAB37,IMP } \\
\text { DH1,ARHGAP45,CST3, } \\
\text { TSPAN14,TNFRSF1B,S } \\
\text { ERPINA1,SLC2A3,ITG } \\
\text { AL,CD47,ITGAM,FTH1, } \\
\text { PKM,TBC1D10C,B4GA } \\
\text { LT1,MAN2B1,STK10,C } \\
\text { MTM6,DOCK2,SLC44A } \\
2\end{array}$ \\
\hline $\begin{array}{l}\text { REAC:R-HSA- } \\
168256\end{array}$ & Immune System & $1.38 \mathrm{E}-09$ & $\begin{array}{l}8.861116 \\
751\end{array}$ & 104 & $\begin{array}{l}\text { LTA,SPSB2,UBOX5,ITG } \\
\text { AX,F13A1,FLNA,TUBB } \\
\text { 1,CD79A,CSF1R,NUP21 } \\
\text { 0,HLA- } \\
\text { A,VCL,PYGB,TREML1, } \\
\text { MLEC,PSAP,CD300E,T } \\
\text { LR5,ACAA1,PTPRJ,P2R } \\
\text { X1,CLU,CIITA,FCN1,IT } \\
\text { GB5,CDKN1A,TKFC,PA } \\
\text { NX1,NCSTN,IRF7,SMA } \\
\text { D3,GLIPR1,CTSA,ITGB } \\
\text { 1,ANO6,CAPN1,IGHM, } \\
\text { TLR2,RNASET2,TCIRG } \\
\text { 1,HLA- } \\
\text { E,ADA2,PTPRC,NBEAL } \\
\text { 2,MYH9,ITGA4,SLA2,C } \\
\text { ARD11,JAK3,BTLA,AP } \\
\text { P,TMEM63A,UNC13D,T } \\
\text { MC6,STIM1,RAB5C,ITP } \\
\text { R1,RNF213,LILRB2,ITG } \\
\text { B2,DAPP1,TNFSF4,RAB }\end{array}$ \\
\hline
\end{tabular}




\begin{tabular}{|c|c|c|c|c|c|}
\hline $\begin{array}{l}\text { REAC:R-HSA- } \\
1912420\end{array}$ & $\begin{array}{l}\text { Pre-NOTCH } \\
\text { Processing in Golgi }\end{array}$ & $\begin{array}{l}0.000492 \\
38\end{array}$ & $\begin{array}{l}3.307699 \\
894\end{array}$ & 6 & $\begin{array}{l}\text { ATP2A3,NOTCH2,LFN } \\
\text { G,NOTCH1,B4GALT1,A } \\
\text { TP2A2 }\end{array}$ \\
\hline $\begin{array}{l}\text { REAC:R-HSA- } \\
194315\end{array}$ & $\begin{array}{l}\text { Signaling by Rho } \\
\text { GTPases }\end{array}$ & $\begin{array}{l}0.011456 \\
442\end{array}$ & $\begin{array}{l}1.940950 \\
253\end{array}$ & 26 & $\begin{array}{l}\text { FLNA,TUBB1,ARHGAP } \\
\text { 27,ITGB1,ARHGAP6,M } \\
\text { YH9,MRTFA,FGD3,MY } \\
\text { LK,CENPT,ARHGAP45, } \\
\text { FGD2,ARHGEF18,TUB } \\
\text { A4A,ARHGEF1,EVL,CT } \\
\text { TN,WAS,ARHGAP18,P } \\
\text { RKCA,ARHGAP4,WIPF } \\
\text { 1,MYO9B,PREX1,PKN1 } \\
\text {,ACTB }\end{array}$ \\
\hline $\begin{array}{l}\text { REAC:R-HSA- } \\
1280215\end{array}$ & $\begin{array}{l}\text { Cytokine Signaling in } \\
\text { Immune system }\end{array}$ & $\begin{array}{l}0.017394 \\
819\end{array}$ & $\begin{array}{l}1.759580 \\
073\end{array}$ & 34 & $\begin{array}{l}\text { LTA,ITGAX,F13A1,FLN } \\
\text { A,CSF1R,NUP210,HLA- } \\
\text { A,PTPRJ,CIITA,CDKN1 } \\
\text { A,IRF7,SMAD3,ITGB1, } \\
\text { HLA- } \\
\text { E,SLA2,JAK3,APP,ITGB } \\
\text { 2,TNFSF4,UBA7,IL17R } \\
\text { A,IL10RA,IL6R,TIMP1, } \\
\text { GRAP2,IL2RG,TNFRSF } \\
\text { 1B,PIK3CD,NUP188,IT } \\
\text { GAM,CSF3R,STXBP2,I } \\
\text { RAK3,PTPN18 }\end{array}$ \\
\hline
\end{tabular}


Table 4 Topology table for up and down regulated genes.

\begin{tabular}{|c|c|c|c|c|c|}
\hline Regulation & Node & Degree & Betweenness & Stress & Closeness \\
\hline Up & TRIM54 & 133 & 0.012147 & 21844832 & 0.322581 \\
\hline Up & ELAVL2 & 118 & 0.009706 & 13548160 & 0.323122 \\
\hline Up & PTN & 109 & 0.008667 & 10295782 & 0.325332 \\
\hline Up & KIT & 105 & 0.005763 & 7684058 & 0.332132 \\
\hline Up & BMPR1B & 102 & 0.012139 & 7911102 & 0.352362 \\
\hline Up & ERRFI1 & 99 & 0.008331 & 11254818 & 0.338466 \\
\hline Up & CCNE1 & 86 & 0.003025 & 5367340 & 0.330825 \\
\hline Up & CCNA1 & 83 & 0.004101 & 5615582 & 0.34443 \\
\hline Up & GLP1R & 78 & 0.010843 & 23727352 & 0.299903 \\
\hline Up & EDA & 74 & 0.008686 & 5992546 & 0.315667 \\
\hline Up & PPARGC1A & 72 & 0.002826 & 4898866 & 0.322485 \\
\hline Up & KRT13 & 70 & 0.004347 & 10153884 & 0.31577 \\
\hline Up & SLC15A1 & 64 & 0.00578 & 5083164 & 0.301448 \\
\hline Up & ANTXR1 & 59 & 0.006338 & 4754724 & 0.364739 \\
\hline Up & ZBTB8A & 59 & 0.00408 & 8024144 & 0.307376 \\
\hline Up & KRT14 & 57 & 0.001607 & 3870640 & 0.322978 \\
\hline Up & NPY2R & 57 & 0.004358 & 4577120 & 0.302607 \\
\hline Up & SLC18A1 & 56 & 0.005946 & 4356010 & 0.306766 \\
\hline Up & LRRC15 & 54 & 0.005085 & 12368332 & 0.298816 \\
\hline Up & PTPN3 & 53 & 0.00338 & 4379752 & 0.322377 \\
\hline $\mathrm{Up}$ & TMPRSS11B & 53 & 0.005232 & 13231264 & 0.28515 \\
\hline Up & TUFT1 & 50 & 0.002391 & 2006556 & 0.308054 \\
\hline Up & BMP7 & 48 & 0.004125 & 3406724 & 0.310316 \\
\hline
\end{tabular}




\begin{tabular}{|c|c|c|c|c|c|}
\hline $\mathrm{Up}$ & MIPOL1 & 47 & 0.003199 & 7831728 & 0.296433 \\
\hline Up & DSG2 & 43 & 0.00103 & 1538692 & 0.326669 \\
\hline Up & MAGEA10 & 42 & 0.002659 & 2782598 & 0.311971 \\
\hline Up & TLR3 & 41 & 0.002523 & 2126014 & 0.311365 \\
\hline Up & MAST1 & 41 & 0.002679 & 1449942 & 0.345502 \\
\hline Up & F9 & 40 & 0.003205 & 3436702 & 0.301448 \\
\hline $\mathrm{Up}$ & DTNA & 39 & 0.003643 & 2835460 & 0.311825 \\
\hline Up & DSG4 & 39 & 0.002547 & 6333108 & 0.286045 \\
\hline Up & LIN28B & 38 & 0.002233 & 2392960 & 0.306429 \\
\hline Up & EPHA3 & 38 & 0.003012 & 1844952 & 0.317553 \\
\hline Up & GDA & 38 & 0.002698 & 4154080 & 0.301995 \\
\hline Up & IL20RA & 37 & 0.003001 & 2163774 & 0.306885 \\
\hline Up & EYA4 & 36 & 0.002123 & 2202158 & 0.300142 \\
\hline Up & CCL22 & 35 & 0.003053 & 4599286 & 0.300548 \\
\hline $\mathrm{Up}$ & SLC1A1 & 35 & 0.004187 & 3161236 & 0.346317 \\
\hline Up & PCDHAC2 & 35 & 0.004863 & 3849886 & 0.292706 \\
\hline Up & IGSF21 & 34 & 0.002215 & 2796766 & 0.296119 \\
\hline Up & SH3RF2 & 34 & 0.001943 & 1330190 & 0.342509 \\
\hline $\mathrm{Up}$ & USP43 & 34 & 0.00183 & 2158278 & 0.305694 \\
\hline Up & COBL & 33 & 7.34E-04 & 789636 & 0.331775 \\
\hline Up & FLG & 33 & 0.001565 & 2103066 & 0.312027 \\
\hline Up & SERPINB13 & 33 & 0.002022 & 2211972 & 0.303009 \\
\hline Up & COLEC12 & 32 & 0.002562 & 1515874 & 0.335126 \\
\hline Up & GFPT2 & 30 & 0.002137 & 2091622 & 0.31094 \\
\hline Up & SFTPC & 30 & 0.002498 & 2902984 & 0.304276 \\
\hline Up & LRP1B & 29 & 0.003228 & 1563906 & 0.344553 \\
\hline
\end{tabular}




\begin{tabular}{|c|c|c|c|c|c|}
\hline Up & AZGP1 & 29 & 0.001511 & 2326868 & 0.30975 \\
\hline Up & ETV1 & 28 & $6.64 \mathrm{E}-04$ & 1570448 & 0.306701 \\
\hline Up & CPA4 & 27 & 0.001782 & 1645444 & 0.303476 \\
\hline Up & KLK11 & 27 & 0.002312 & 3332010 & 0.2924 \\
\hline Up & CTNNA2 & 27 & 0.001317 & 2711166 & 0.312714 \\
\hline Up & WNT5A & 27 & 0.002257 & 1865676 & 0.296525 \\
\hline Up & KRT85 & 27 & 0.001319 & 2630340 & 0.305975 \\
\hline Up & FAP & 27 & 0.001924 & 3392714 & 0.291986 \\
\hline Up & ROBO2 & 26 & 0.001435 & 2365116 & 0.291651 \\
\hline Up & KCNIP3 & 26 & 0.001472 & 3374664 & 0.28202 \\
\hline Up & BRINP1 & 25 & 0.0012 & 2988948 & 0.301301 \\
\hline Up & NCCRP1 & 25 & $8.25 \mathrm{E}-04$ & 1228816 & 0.315173 \\
\hline Up & ART3 & 24 & 0.001611 & 3187858 & 0.283016 \\
\hline Up & INAVA & 24 & 0.00154 & 1886482 & 0.291278 \\
\hline Up & CREB3L3 & 24 & 0.001636 & 2012464 & 0.302332 \\
\hline Up & SLIT2 & 23 & 0.001176 & 1918318 & 0.301259 \\
\hline Up & POPDC2 & 23 & 0.002173 & 1488558 & 0.271797 \\
\hline Up & IZUMO1 & 23 & 0.002732 & 4118726 & 0.259647 \\
\hline Up & MAL2 & 23 & 0.001473 & 2898432 & 0.296261 \\
\hline Up & RGPD8 & 22 & 0.001517 & 1034252 & 0.305586 \\
\hline Up & PAEP & 22 & 0.001035 & 874582 & 0.343828 \\
\hline Up & ASB5 & 22 & $4.24 \mathrm{E}-04$ & 368080 & 0.303583 \\
\hline Up & FAM83B & 22 & $8.25 \mathrm{E}-04$ & 1549462 & 0.305166 \\
\hline Up & SLC5A8 & 22 & 0.001364 & 1608962 & 0.276598 \\
\hline Up & SIX1 & 21 & 0.00131 & 1136652 & 0.295735 \\
\hline Up & KCND2 & 21 & 0.002331 & 1658754 & 0.303956 \\
\hline
\end{tabular}




\begin{tabular}{|c|c|c|c|c|c|}
\hline $\mathrm{Up}$ & MYH4 & 21 & $9.70 \mathrm{E}-04$ & 980960 & 0.299623 \\
\hline Up & NEUROD1 & 21 & 0.001441 & 2678376 & 0.287985 \\
\hline Up & PAK3 & 20 & $6.35 \mathrm{E}-05$ & 179392 & 0.297748 \\
\hline Up & TACC2 & 20 & 0.001037 & 1183890 & 0.309374 \\
\hline Up & TTC25 & 20 & 0.001315 & 2071982 & 0.276139 \\
\hline Up & LBP & 19 & 0.001144 & 837746 & 0.309917 \\
\hline $\mathrm{Up}$ & HRG & 19 & $8.05 \mathrm{E}-04$ & 1333666 & 0.292607 \\
\hline Up & CDC20B & 19 & 0.001052 & 1653682 & 0.279211 \\
\hline Up & TNR & 19 & 0.001002 & 1524772 & 0.286092 \\
\hline Up & SHROOM3 & 18 & $6.22 \mathrm{E}-04$ & 712586 & 0.316393 \\
\hline $\mathrm{Up}$ & PHKA1 & 18 & $8.26 \mathrm{E}-04$ & 664362 & 0.301763 \\
\hline Up & RIMS2 & 18 & $8.17 \mathrm{E}-04$ & 1001820 & 0.305575 \\
\hline Up & CSRP2 & 18 & $2.40 \mathrm{E}-04$ & 457510 & 0.304983 \\
\hline Up & KRT24 & 18 & $2.78 \mathrm{E}-05$ & 166448 & 0.30059 \\
\hline $\mathrm{Up}$ & LRRC7 & 18 & $6.50 \mathrm{E}-04$ & 1043008 & 0.299085 \\
\hline Up & ITGA8 & 17 & 0.001062 & 974108 & 0.291524 \\
\hline Up & SIGLEC5 & 17 & 0.001041 & 718852 & 0.290351 \\
\hline Up & CLDN1 & 17 & 7.91E-04 & 1036670 & 0.284009 \\
\hline $\mathrm{Up}$ & IGF1 & 17 & 0.001485 & 2375614 & 0.275937 \\
\hline Up & MYF6 & 17 & $3.75 \mathrm{E}-04$ & 472100 & 0.284503 \\
\hline Up & CDH8 & 17 & $7.20 \mathrm{E}-04$ & 923698 & 0.293351 \\
\hline Up & TRIM71 & 17 & $9.54 \mathrm{E}-04$ & 1482892 & 0.250325 \\
\hline Up & ESRP1 & 16 & $9.05 \mathrm{E}-04$ & 638852 & 0.286983 \\
\hline Up & GPC6 & 16 & 0.001651 & 1480508 & 0.325674 \\
\hline Up & CPEB1 & 16 & 0.001555 & 915000 & 0.327906 \\
\hline $\mathrm{Up}$ & GRIA4 & 16 & $3.00 \mathrm{E}-04$ & 243164 & 0.303519 \\
\hline
\end{tabular}




\begin{tabular}{|c|c|c|c|c|c|}
\hline $\mathrm{Up}$ & ID4 & 16 & $9.00 \mathrm{E}-04$ & 532420 & 0.307977 \\
\hline Up & TRPV6 & 16 & $6.17 \mathrm{E}-04$ & 609086 & 0.281005 \\
\hline Up & GJC1 & 16 & 0.001409 & 1565962 & 0.27087 \\
\hline Up & FERMT1 & 16 & $4.60 \mathrm{E}-04$ & 808642 & 0.300267 \\
\hline Up & EFHD1 & 16 & $9.97 \mathrm{E}-04$ & 2285970 & 0.281553 \\
\hline Up & ZMAT4 & 15 & 0.00194 & 2108242 & 0.331141 \\
\hline $\mathrm{Up}$ & FUT3 & 15 & 0.001643 & 1729770 & 0.26295 \\
\hline Up & KIR3DL1 & 15 & $6.23 \mathrm{E}-04$ & 1269024 & 0.278188 \\
\hline Up & MC1R & 15 & 0.001048 & 1221034 & 0.282739 \\
\hline Up & BNIPL & 15 & $9.69 \mathrm{E}-04$ & 1242002 & 0.277315 \\
\hline $\mathrm{Up}$ & PHYHIPL & 15 & $4.86 \mathrm{E}-04$ & 372398 & 0.288465 \\
\hline Up & PCDHB7 & 15 & 0.00158 & 1015798 & 0.272361 \\
\hline Up & IGSF1 & 14 & 7.87E-04 & 482056 & 0.268635 \\
\hline Up & ST8SIA3 & 14 & 8.32E-04 & 665984 & 0.291543 \\
\hline $\mathrm{Up}$ & CA12 & 14 & $6.44 \mathrm{E}-05$ & 102792 & 0.268619 \\
\hline Up & SCEL & 14 & $7.34 \mathrm{E}-04$ & 388298 & 0.33479 \\
\hline Up & SLC34A2 & 14 & 0.001583 & 1563014 & 0.247542 \\
\hline Up & GABRB3 & 14 & $5.08 \mathrm{E}-04$ & 274876 & 0.300924 \\
\hline $\mathrm{Up}$ & ANKS1B & 4 & $4.96 \mathrm{E}-05$ & 207240 & 0.331775 \\
\hline Up & CIART & 2 & $5.70 \mathrm{E}-05$ & 275826 & 0.319652 \\
\hline Up & $\mathrm{IQCH}$ & 2 & 0 & 0 & 0.31964 \\
\hline Up & CXCL14 & 2 & $3.86 \mathrm{E}-05$ & 97804 & 0.322749 \\
\hline Up & LONRF2 & 1 & 0 & 0 & 0.2311 \\
\hline Up & ITIH3 & 1 & 0 & 0 & 0.243265 \\
\hline Up & DACT3 & 1 & 0 & 0 & 0.240369 \\
\hline Up & DECR 2 & 1 & 0 & 0 & 0.317752 \\
\hline
\end{tabular}




\begin{tabular}{|c|c|c|c|c|c|}
\hline Up & CFAP53 & 1 & 0 & 0 & 0.317752 \\
\hline Up & NDP & 1 & 0 & 0 & 0.317752 \\
\hline Up & ANKRD45 & 1 & 0 & 0 & 0.317752 \\
\hline Up & CNTN4 & 1 & 0 & 0 & 0.317752 \\
\hline Up & GRHL2 & 1 & 0 & 0 & 0.317752 \\
\hline Up & STOML1 & 1 & 0 & 0 & 0.317752 \\
\hline Up & RBPJL & 1 & 0 & 0 & 0.317752 \\
\hline Up & ERVFRD-1 & 1 & 0 & 0 & 0.317752 \\
\hline Up & SHC4 & 1 & 0 & 0 & 0.249331 \\
\hline Up & SLC9A2 & 1 & 0 & 0 & 0.28788 \\
\hline Up & RASEF & 1 & 0 & 0 & 0.247252 \\
\hline Up & SVOP & 1 & 0 & 0 & 0.230718 \\
\hline Up & SLC35F1 & 1 & 0 & 0 & 0.232315 \\
\hline Up & CELF5 & 1 & 0 & 0 & 0.244219 \\
\hline Up & ZNF599 & 1 & 0 & 0 & 0.232066 \\
\hline Up & C5orf34 & 1 & 0 & 0 & 0.240823 \\
\hline Up & ROS1 & 1 & 0 & 0 & 0.279842 \\
\hline Up & CRISP2 & 1 & 0 & 0 & 0.269028 \\
\hline Up & ASPA & 1 & 0 & 0 & 0.239909 \\
\hline Up & CLDN12 & 1 & 0 & 0 & 0.246359 \\
\hline Up & RSPO1 & 1 & 0 & 0 & 0.256266 \\
\hline Up & WNK3 & 1 & 0 & 0 & 0.247598 \\
\hline Up & FRMPD1 & 1 & 0 & 0 & 0.260037 \\
\hline Up & DRP2 & 1 & 0 & 0 & 0.23771 \\
\hline Down & APP & 2189 & 0.002666 & 3365310 & 0.317845 \\
\hline Down & SRC & 504 & 0.400224 & $5.59 \mathrm{E}+08$ & 0.465717 \\
\hline
\end{tabular}




\begin{tabular}{|c|c|c|c|c|c|}
\hline Down & ITGA4 & 496 & 0.008595 & 8040232 & 0.303306 \\
\hline Down & RPA1 & 493 & 0 & 0 & 0.2311 \\
\hline Down & АCTB & 476 & 0.01213 & 21489770 & 0.335607 \\
\hline Down & SMAD3 & 398 & 0.010658 & 16678612 & 0.357999 \\
\hline Down & MYH9 & 364 & 0.006158 & 6598872 & 0.337227 \\
\hline Down & FLNA & 353 & 0.007077 & 11964634 & 0.337885 \\
\hline Down & PKM & 353 & $4.21 \mathrm{E}-05$ & 68424 & 0.292597 \\
\hline Down & CDKN1A & 302 & 0.010037 & 16637086 & 0.367027 \\
\hline Down & TUBA4A & 281 & 0.004054 & 3547868 & 0.316706 \\
\hline Down & PRKCA & 279 & 4.19E-05 & 33780 & 0.274616 \\
\hline Down & SRRM2 & 259 & 0.022533 & 38294108 & 0.381347 \\
\hline Down & NOTCH1 & 245 & 0.013646 & 27956328 & 0.345433 \\
\hline Down & VCL & 233 & 0.005656 & 4112886 & 0.355311 \\
\hline Down & CDK9 & 211 & 0.031272 & 43724982 & 0.387802 \\
\hline Down & UPF1 & 199 & 0.048906 & $1.46 \mathrm{E}+08$ & 0.371865 \\
\hline Down & MEPCE & 186 & 0.005625 & 5736504 & 0.361481 \\
\hline Down & LGALS3BP & 176 & 0.050731 & 56826626 & 0.397302 \\
\hline Down & ACTN1 & 172 & 0.005719 & 8627714 & 0.316335 \\
\hline Down & $\mathrm{CAD}$ & 171 & 0.010289 & 9147932 & 0.323472 \\
\hline Down & TLN1 & 170 & 0.024835 & 32927066 & 0.377671 \\
\hline Down & RARA & 168 & 0.016376 & 38790824 & 0.34524 \\
\hline Down & RAB5C & 164 & 0.009051 & 26501322 & 0.32746 \\
\hline Down & E2F3 & 157 & 0.067172 & 81164158 & 0.404238 \\
\hline Down & MYBBP1A & 157 & 0.004253 & 4853872 & 0.319216 \\
\hline Down & SF3A2 & 153 & 0.005345 & 5537860 & 0.321455 \\
\hline Down & NCSTN & 148 & 0.013675 & 11620366 & 0.373614 \\
\hline
\end{tabular}




\begin{tabular}{|c|c|c|c|c|c|}
\hline Down & PLEC & 148 & 0.007198 & 7735012 & 0.333667 \\
\hline Down & CLU & 148 & 0.004284 & 4921862 & 0.315621 \\
\hline Down & CTTN & 144 & $1.85 \mathrm{E}-05$ & 34480 & 0.273479 \\
\hline Down & LRP1 & 143 & 0.035606 & 66738612 & 0.368025 \\
\hline Down & ITGB1 & 141 & 0.007016 & 11423404 & 0.330433 \\
\hline Down & OGT & 139 & 0.017531 & 34670330 & 0.363911 \\
\hline Down & NCOR2 & 136 & 0.05242 & 67476208 & 0.414314 \\
\hline Down & TNIK & 133 & 0.003794 & 7500172 & 0.323255 \\
\hline Down & CAPN1 & 132 & 0.011955 & 9201942 & 0.362783 \\
\hline Down & TNFRSF1B & 126 & 0.005235 & 6048582 & 0.338347 \\
\hline Down & RAB8A & 126 & 0.033442 & 45967308 & 0.398711 \\
\hline Down & ZYX & 122 & 0.002615 & 4159134 & 0.316671 \\
\hline Down & TGFB1 & 121 & 0.030548 & 55611552 & 0.380726 \\
\hline Down & GANAB & 121 & 0.004766 & 9084764 & 0.316324 \\
\hline Down & FTH1 & 112 & 0 & 0 & 0.240315 \\
\hline Down & FTSJ1 & 110 & 0.008563 & 21677110 & 0.350069 \\
\hline Down & WAS & 105 & 0.006824 & 14951804 & 0.316416 \\
\hline Down & ATP2A2 & 104 & 0.005502 & 13512860 & 0.325454 \\
\hline Down & BAX & 103 & 0.002749 & 7775802 & 0.312997 \\
\hline Down & SNRPA & 103 & 0.004686 & 7615722 & 0.323146 \\
\hline Down & FOXK1 & 103 & $6.56 \mathrm{E}-05$ & 124624 & 0.297523 \\
\hline Down & CBX6 & 102 & $2.60 \mathrm{E}-05$ & 130320 & 0.300101 \\
\hline Down & HDAC7 & 100 & $7.36 \mathrm{E}-06$ & 41282 & 0.289409 \\
\hline Down & KRAS & 100 & 0.00505 & 3946654 & 0.314372 \\
\hline Down & TUBB1 & 99 & 0.010762 & 7334820 & 0.356496 \\
\hline Down & USP39 & 99 & 0.004163 & 4124064 & 0.333346 \\
\hline
\end{tabular}




\begin{tabular}{|c|c|c|c|c|c|}
\hline Down & STRN4 & 98 & 0.015639 & 13083920 & 0.371546 \\
\hline Down & GDI1 & 97 & 0.00808 & 7245854 & 0.355151 \\
\hline Down & POLR1A & 95 & 0.004493 & 3158464 & 0.315356 \\
\hline Down & GIGYF1 & 94 & 0.00389 & 8518854 & 0.317868 \\
\hline Down & MED12 & 94 & $1.58 \mathrm{E}-05$ & 10046 & 0.275586 \\
\hline Down & JAK3 & 93 & 0.011564 & 8350784 & 0.351704 \\
\hline Down & ENO2 & 92 & 0 & 0 & 0.260201 \\
\hline Down & RAB1B & 89 & 0.008892 & 17406094 & 0.336899 \\
\hline Down & CTDSPL & 86 & 0.005896 & 10405078 & 0.326792 \\
\hline Down & GRAP2 & 84 & 0.010691 & 20962628 & 0.34186 \\
\hline Down & TSC22D4 & 84 & 0.008449 & 16411146 & 0.350849 \\
\hline Down & PTPRJ & 83 & 0.003374 & 6154730 & 0.317449 \\
\hline Down & CXCR4 & 82 & 0 & 0 & 0.319392 \\
\hline Down & MDM4 & 81 & 0 & 0 & 0.321014 \\
\hline Down & SRSF6 & 81 & 0.00369 & 2617526 & 0.348744 \\
\hline Down & IMPDH1 & 79 & 0.008468 & 14872056 & 0.324636 \\
\hline Down & PTPRC & 78 & 0 & 0 & 0.245083 \\
\hline Down & CLPP & 78 & 0 & 0 & 0.317752 \\
\hline Down & TLR2 & 77 & 4.79E-05 & 76992 & 0.322317 \\
\hline Down & CORO1B & 77 & 0 & 0 & 0.317752 \\
\hline Down & USP22 & 76 & 0 & 0 & 0.317752 \\
\hline Down & SIN3B & 76 & 0.006673 & 5072566 & 0.357084 \\
\hline Down & SUN2 & 75 & 0 & 0 & 0.319369 \\
\hline Down & PI4KA & 75 & 5.09E-05 & 194840 & 0.320231 \\
\hline Down & MRPL28 & 75 & 0 & 0 & 0.317752 \\
\hline Down & IL17RA & 75 & 0 & 0 & 0.317752 \\
\hline
\end{tabular}




\begin{tabular}{|c|c|c|c|c|c|}
\hline Down & PKN1 & 74 & 0 & 0 & 0.317752 \\
\hline Down & USP20 & 74 & 0.003156 & 4712314 & 0.357777 \\
\hline Down & CHERP & 73 & $1.58 \mathrm{E}-04$ & 227708 & 0.320717 \\
\hline Down & VPS52 & 73 & $5.49 \mathrm{E}-05$ & 172518 & 0.322077 \\
\hline Down & TGOLN2 & 71 & 0 & 0 & 0.317752 \\
\hline Down & G6PD & 70 & $9.99 \mathrm{E}-05$ & 528874 & 0.329578 \\
\hline Down & POMK & 69 & 0 & 0 & 0.317752 \\
\hline Down & DENND4C & 69 & $4.16 \mathrm{E}-05$ & 440608 & 0.329353 \\
\hline Down & PRKAR2B & 69 & 0 & 0 & 0.317752 \\
\hline Down & ITGB3 & 66 & 0 & 0 & 0.317752 \\
\hline Down & PAX5 & 65 & $9.66 \mathrm{E}-05$ & 409222 & 0.327385 \\
\hline Down & ITGB5 & 64 & 0 & 0 & 0.317752 \\
\hline Down & NOTCH2 & 62 & 0.0076 & 4500746 & 0.353023 \\
\hline Down & SERPINA1 & 61 & 0 & 0 & 0.317752 \\
\hline Down & RNF166 & 61 & 0 & 0 & 0.317752 \\
\hline Down & LEF1 & 60 & 0.002734 & 2878552 & 0.356893 \\
\hline Down & RUFY1 & 59 & 0 & 0 & 0.317752 \\
\hline Down & ITPR1 & 59 & 0.016504 & 15009770 & 0.367541 \\
\hline Down & IRF7 & 58 & $4.20 \mathrm{E}-05$ & 254816 & 0.325797 \\
\hline Down & CDK2AP1 & 58 & 0.016675 & 15745286 & 0.373937 \\
\hline Down & THBS1 & 57 & 0 & 0 & 0.317752 \\
\hline Down & HLA-E & 57 & 0.007079 & 6193594 & 0.359694 \\
\hline Down & RNH1 & 56 & 5.89E-05 & 307276 & 0.322725 \\
\hline Down & ELAC2 & 56 & 0.006031 & 7284664 & 0.360863 \\
\hline Down & PACSIN2 & 55 & 0 & 0 & 0.318476 \\
\hline Down & ARL6IP5 & 55 & 0 & 0 & 0.317752 \\
\hline
\end{tabular}




\begin{tabular}{|c|c|c|c|c|c|}
\hline Down & CCNL2 & 55 & 0 & 0 & 0.317752 \\
\hline Down & ATP2A3 & 55 & 0.033293 & 57837312 & 0.388567 \\
\hline Down & DIMT1 & 54 & 0 & 0 & 0.317752 \\
\hline Down & PSAP & 54 & 0 & 0 & 0.317752 \\
\hline Down & CD79A & 54 & $3.27 \mathrm{E}-04$ & 585398 & 0.330711 \\
\hline Down & VPS51 & 54 & 0 & 0 & 0.317752 \\
\hline Down & CTDNEP1 & 53 & 0 & 0 & 0.321443 \\
\hline Down & ITGB2 & 53 & 0.002039 & 3160000 & 0.334208 \\
\hline Down & LIMS1 & 53 & 0.009901 & 17849898 & 0.334376 \\
\hline Down & CMTM5 & 53 & 0 & 0 & 0.279878 \\
\hline Down & GNB5 & 52 & 0.042962 & $1.29 \mathrm{E}+08$ & 0.371403 \\
\hline Down & ESYT1 & 52 & 0.010431 & 19740350 & 0.351404 \\
\hline Down & EVL & 52 & 0.002936 & 3873720 & 0.321861 \\
\hline Down & PYGB & 50 & 0 & 0 & 0.243498 \\
\hline Down & HLA-A & 7 & $3.15 \mathrm{E}-06$ & 4282 & 0.25335 \\
\hline Down & CSF1R & 4 & 0 & 0 & 0.243498 \\
\hline Down & MYLK & 4 & 0.02758 & 53431176 & 0.357349 \\
\hline Down & MICAL1 & 4 & 0 & 0 & 0.263631 \\
\hline Down & ZВTB7B & 3 & 0.011364 & 18936744 & 0.356555 \\
\hline Down & RIN3 & 3 & 0 & 0 & 0.28158 \\
\hline Down & SPARC & 3 & $5.99 \mathrm{E}-05$ & 100996 & 0.279112 \\
\hline Down & LFNG & 3 & 0 & 0 & 0.25378 \\
\hline Down & PRAM1 & 3 & 0.007566 & 10658530 & 0.33669 \\
\hline Down & FERMT3 & 3 & 0.004345 & 6463622 & 0.323183 \\
\hline Down & APLP2 & 3 & 0.002097 & 5599528 & 0.312207 \\
\hline Down & ACAD10 & 3 & $5.54 \mathrm{E}-06$ & 5790 & 0.281452 \\
\hline
\end{tabular}




\begin{tabular}{|c|c|c|c|c|c|}
\hline Down & ITGA2B & 3 & 0.003888 & 5735454 & 0.329328 \\
\hline Down & VCAN & 3 & 0.005452 & 8532800 & 0.331116 \\
\hline Down & LENG8 & 3 & 0.005412 & 6353682 & 0.338466 \\
\hline Down & EPM2AIP1 & 3 & 0.004426 & 3470384 & 0.316764 \\
\hline Down & MAP1A & 3 & 0.00816 & 16680766 & 0.33231 \\
\hline Down & STON2 & 3 & 0.003943 & 9629562 & 0.331737 \\
\hline Down & $\mathrm{ABI} 3$ & 3 & $6.24 \mathrm{E}-05$ & 141136 & 0.260663 \\
\hline Down & TRIM13 & 3 & 0.006012 & 17818956 & 0.328279 \\
\hline Down & WIPF1 & 3 & 0.006612 & 10493584 & 0.327423 \\
\hline Down & IL2RG & 3 & 0 & 0 & 0.249331 \\
\hline Down & B4GALT1 & 3 & 0.004053 & 8509232 & 0.32549 \\
\hline Down & DOCK8 & 2 & 0.008903 & 15185354 & 0.333205 \\
\hline Down & NUP188 & 2 & 0.007135 & 7672290 & 0.329065 \\
\hline Down & MAP4K1 & 2 & 0.003653 & 4056316 & 0.316949 \\
\hline Down & PLXDC2 & 2 & $2.45 \mathrm{E}-06$ & 6914 & 0.241879 \\
\hline Down & RASGRP2 & 2 & 0.004447 & 3796740 & 0.322389 \\
\hline Down & CCNL1 & 2 & 0 & 0 & 0.244253 \\
\hline Down & CASP6 & 2 & $3.41 \mathrm{E}-05$ & 75570 & 0.268802 \\
\hline Down & C15orf39 & 2 & 0.008306 & 17301536 & 0.332017 \\
\hline Down & MBNL1 & 2 & $1.36 \mathrm{E}-05$ & 74714 & 0.306896 \\
\hline Down & ZC3HAV1L & 2 & 0.004666 & 6660660 & 0.327695 \\
\hline Down & SMG5 & 2 & $2.15 \mathrm{E}-05$ & 101210 & 0.303529 \\
\hline Down & MRPL49 & 2 & $1.78 \mathrm{E}-05$ & 51682 & 0.289293 \\
\hline Down & LILRB2 & 2 & 0 & 0 & 0.28788 \\
\hline Down & ITGAL & 2 & 0 & 0 & 0.28788 \\
\hline Down & CD47 & 2 & 0 & 0 & 0.28788 \\
\hline
\end{tabular}


Table 5 miRNA - target gene and TF - target gene interaction

\begin{tabular}{|c|c|c|c|c|c|c|c|}
\hline Regulation & $\begin{array}{l}\text { Target } \\
\text { Genes }\end{array}$ & Degree & MicroRNA & Regulation & $\begin{array}{l}\text { Target } \\
\text { Genes }\end{array}$ & Degree & TF \\
\hline $\mathrm{Up}$ & ERRFI1 & 70 & $\begin{array}{l}\text { hsa-mir- } \\
198\end{array}$ & UP & ERRFI1 & 43 & CREM \\
\hline $\mathrm{Up}$ & CCNE1 & 50 & $\begin{array}{l}\text { hsa-mir- } \\
181 b-5 p\end{array}$ & UP & ELAVL2 & 41 & BMI1 \\
\hline $\mathrm{Up}$ & ELAVL2 & 43 & $\begin{array}{l}\text { hsa-mir- } \\
766-3 p\end{array}$ & UP & KIT & 34 & DMRT1 \\
\hline $\mathrm{Up}$ & ANTXR1 & 36 & $\begin{array}{l}\text { hsa-mir- } \\
624-5 p\end{array}$ & UP & ZBTB8A & 29 & GATA4 \\
\hline Up & ZBTB8A & 34 & $\begin{array}{l}\text { hsa-mir- } \\
10 \mathrm{~b}-5 \mathrm{p}\end{array}$ & UP & PPARGC1A & 27 & FOXA2 \\
\hline $\mathrm{Up}$ & KIT & 29 & $\begin{array}{l}\text { hsa-mir- } \\
550 a-5 p\end{array}$ & UP & PTN & 27 & SOX 2 \\
\hline $\mathrm{Up}$ & PPARGC1A & 19 & $\begin{array}{l}\text { hsa-mir- } \\
941\end{array}$ & UP & CCNE1 & 26 & SIN3A \\
\hline Up & CCNA1 & 18 & $\begin{array}{l}\text { hsa-mir- } \\
124-3 p\end{array}$ & UP & ANTXR1 & 26 & SOX9 \\
\hline $\mathrm{Up}$ & PTN & 12 & $\begin{array}{l}\text { hsa-mir- } \\
577\end{array}$ & UP & BMPR1B & 25 & TCF3 \\
\hline $\mathrm{Up}$ & KRT14 & 11 & $\begin{array}{l}\text { hsa-mir- } \\
146 a-5 p\end{array}$ & UP & SLC15A1 & 22 & POU5F1 \\
\hline $\mathrm{Up}$ & BMPR1B & 7 & $\begin{array}{l}\text { hsa-mir- } \\
200 c-3 p\end{array}$ & UP & GLP1R & 20 & GATA1 \\
\hline Up & EDA & 7 & $\begin{array}{l}\text { hsa-mir-16- } \\
5 \mathrm{p}\end{array}$ & UP & CCNA1 & 14 & MYC \\
\hline Up & KRT13 & 5 & $\begin{array}{l}\text { hsa-mir- } \\
1343-3 p\end{array}$ & UP & KRT13 & 13 & SOX17 \\
\hline $\mathrm{Up}$ & TRIM54 & 3 & $\begin{array}{l}\text { hsa-mir- } \\
302 a-3 p\end{array}$ & UP & EDA & 11 & RUNX2 \\
\hline
\end{tabular}




\begin{tabular}{|c|c|c|c|c|c|c|c|}
\hline Up & GLP1R & 1 & $\begin{array}{l}\text { hsa-mir- } \\
376 c-3 p\end{array}$ & UP & TRIM54 & 7 & TEAD4 \\
\hline Down & SRRM2 & 250 & $\begin{array}{l}\text { hsa-mir- } \\
582-5 p\end{array}$ & Down & SMAD3 & 58 & EP300 \\
\hline Down & CDKN1A & 187 & $\begin{array}{c}\text { hsa-mir- } \\
657\end{array}$ & Down & CDKN1A & 57 & STAT3 \\
\hline Down & MYH9 & 126 & $\begin{array}{c}\text { hsa-mir- } \\
1231\end{array}$ & Down & ACTB & 56 & HOXC9 \\
\hline Down & APP & 125 & $\begin{array}{l}\text { hsa-mir- } \\
509-3-5 p\end{array}$ & Down & PKM & 55 & FOXP1 \\
\hline Down & ACTB & 122 & $\begin{array}{c}\text { hsa-mir- } \\
\text { 192-3p }\end{array}$ & Down & MYH9 & 53 & ATF3 \\
\hline Down & VCL & 95 & $\begin{array}{c}\text { hsa-mir- } \\
\text { 590-5p }\end{array}$ & Down & $\mathrm{SRC}$ & 46 & TCF7 \\
\hline Down & FLNA & 92 & $\begin{array}{l}\text { hsa-mir- } \\
\text { 99b-5p }\end{array}$ & Down & NOTCH1 & 46 & RUNX1 \\
\hline Down & PKM & 81 & $\begin{array}{l}\text { hsa-mir- } \\
514 a-3 p\end{array}$ & Down & APP & 45 & TET1 \\
\hline Down & SMAD3 & 56 & $\begin{array}{l}\text { hsa-mir- } \\
\text { 320d }\end{array}$ & Down & VCL & 45 & ESRRB \\
\hline Down & RPA1 & 41 & $\begin{array}{l}\text { hsa-mir- } \\
\text { 196a-3p }\end{array}$ & Down & PRKCA & 43 & MITF \\
\hline Down & NOTCH1 & 35 & $\begin{array}{l}\text { hsa-mir- } \\
1301-3 p\end{array}$ & Down & SRRM2 & 37 & CEBPB \\
\hline Down & PRKCA & 35 & $\begin{array}{c}\text { hsa-mir- } \\
484\end{array}$ & Down & TUBA4A & 24 & SCLY \\
\hline Down & SRC & 27 & $\begin{array}{c}\text { hsa-mir- } \\
4525\end{array}$ & Down & ITGA4 & 19 & GATA1 \\
\hline Down & TUBA4A & 27 & $\begin{array}{l}\text { hsa-mir- } \\
\text { 3619-5p }\end{array}$ & Down & FLNA & 18 & CUX1 \\
\hline Down & ITGA4 & 13 & $\begin{array}{c}\text { hsa-mir- } \\
101-3 p\end{array}$ & Down & RPA1 & 16 & SMARCA4 \\
\hline
\end{tabular}




\section{Figures}
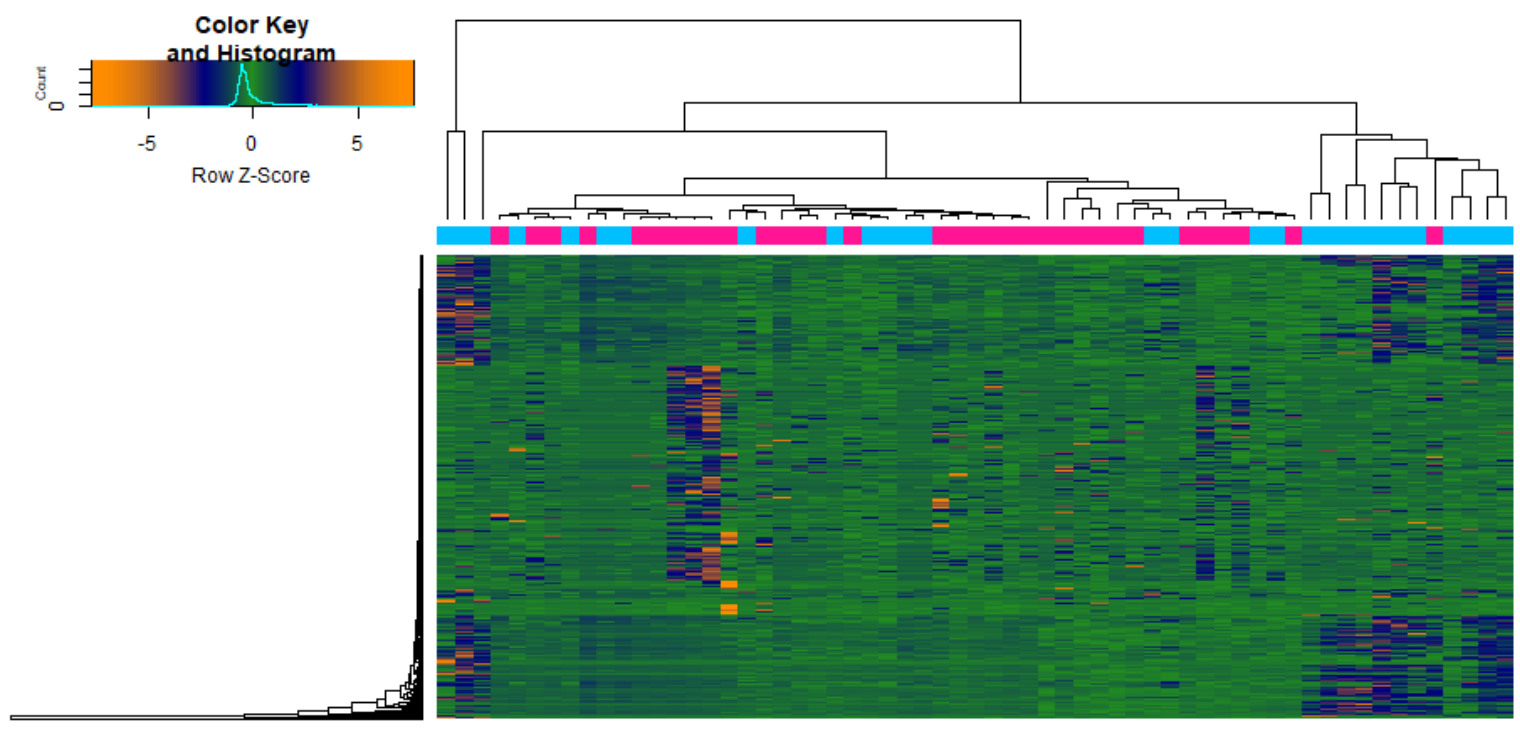

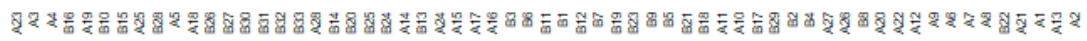

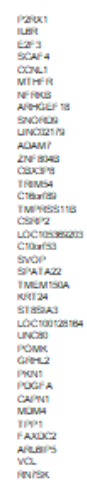

Fig. 1. Heat map of differentially expressed genes. Legend on the top left indicate log fold change of genes. (A1 A28= Normal Pregnancy Samples; B1 - B33= Gestational diabetes Samples)

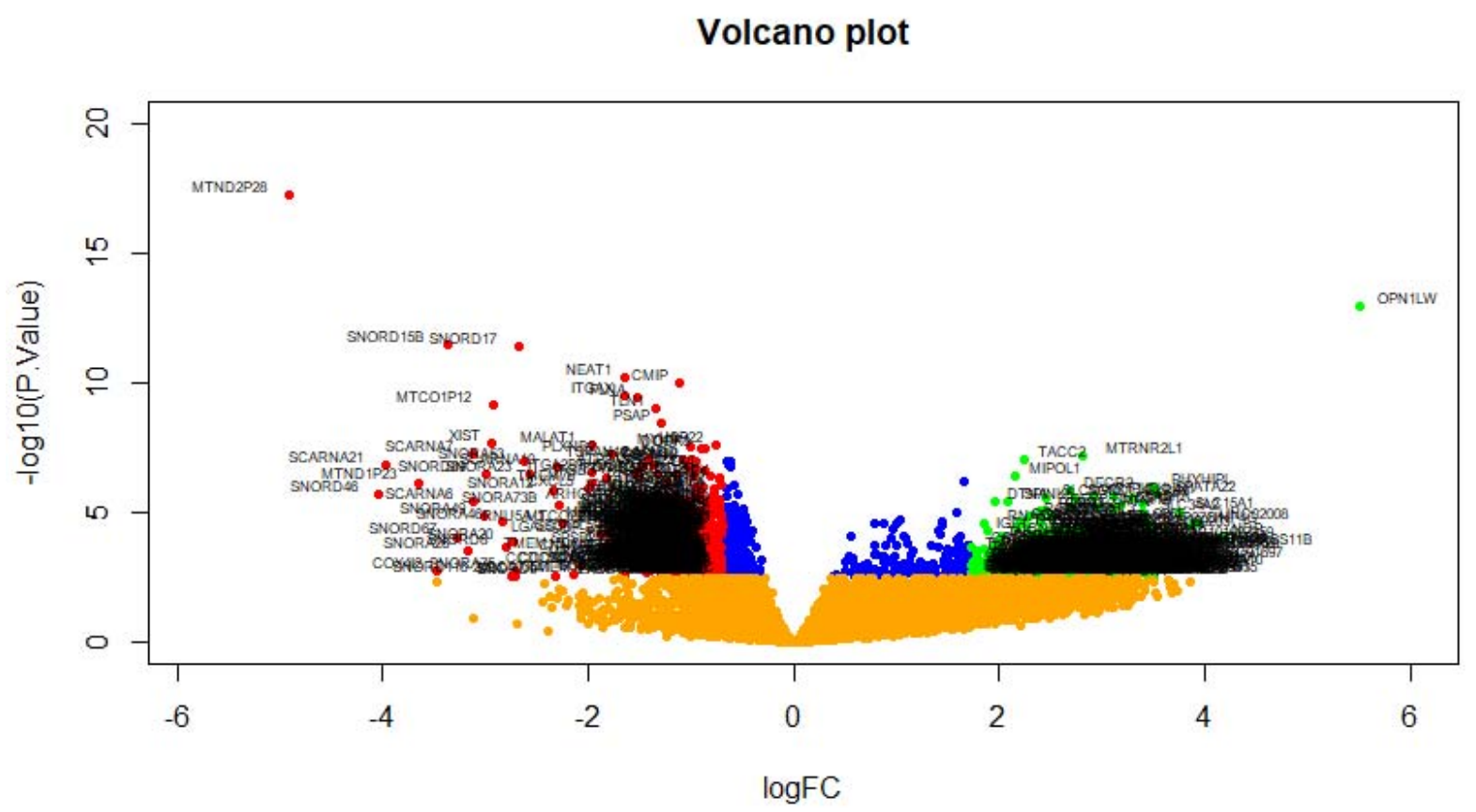


Fig. 2. Volcano plot of differentially expressed genes. Genes with a significant change of more than two-fold were selected. Green dot represented up regulated significant genes and red dot represented down regulated significant genes.

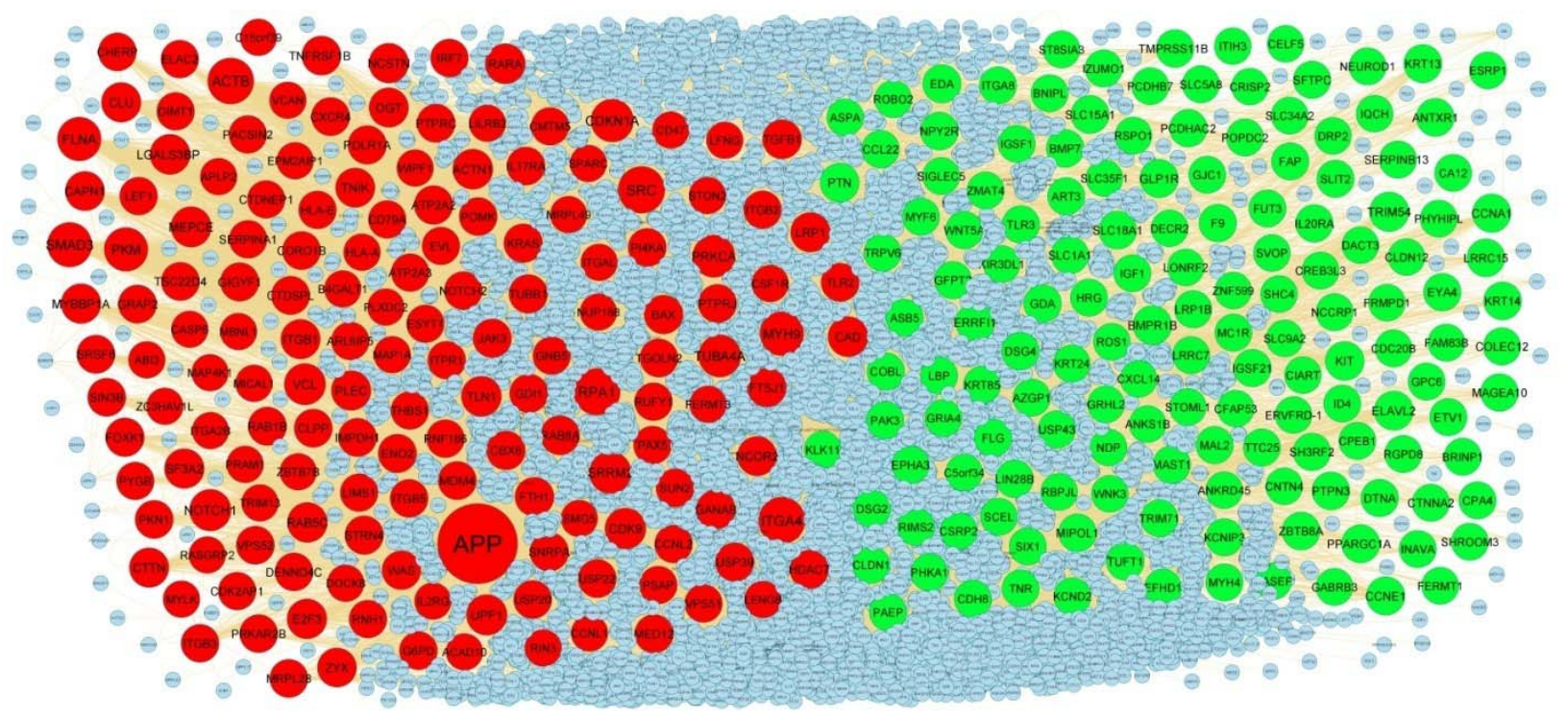

Fig. 3. PPI network of DEGs. Up regulated genes are marked in green; down regulated genes are marked in red
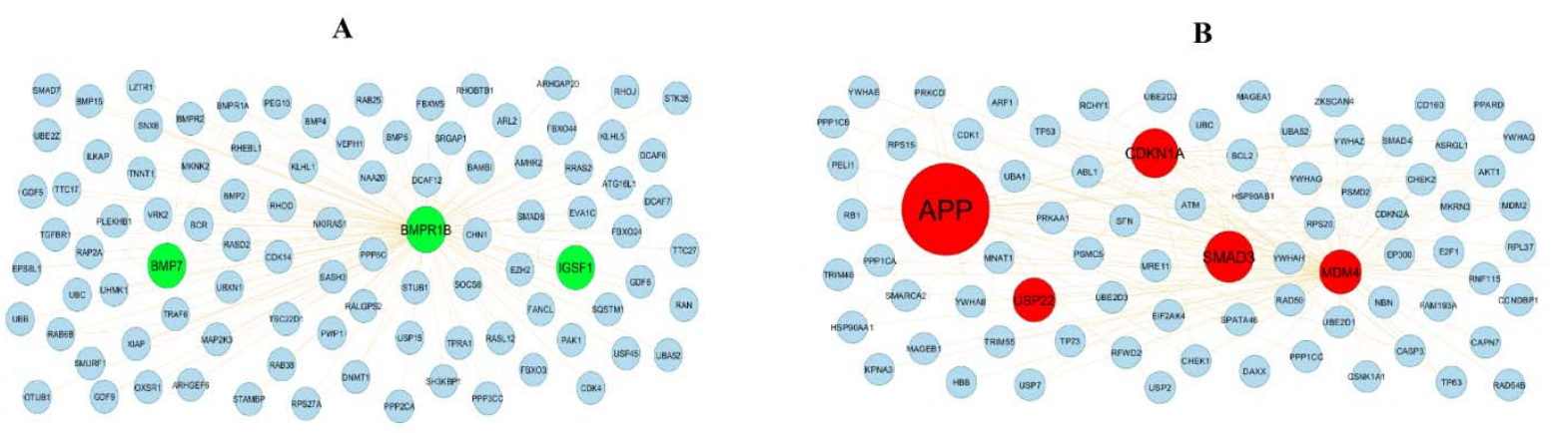

Fig. 4. Modules of isolated form PPI of DEGs. (A) The most significant module was obtained from PPI network with 95 nodes and 103 edges for up regulated genes (B) The most significant module was obtained from PPI network with 79 nodes and 131 edges for down regulated genes. Up regulated genes are marked in green; down regulated genes are marked in red 


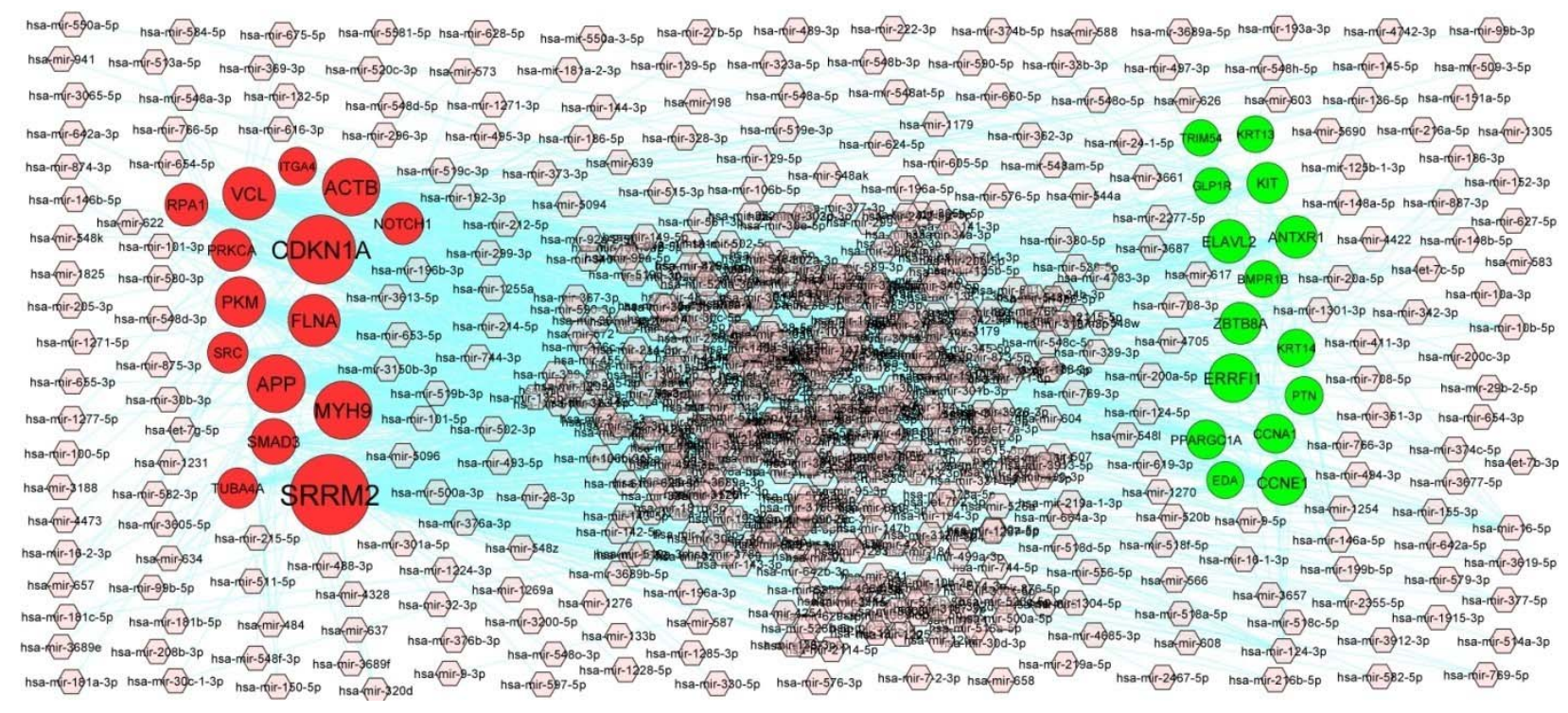

Fig. 5. Target gene - miRNA regulatory network between target genes. The blue color diamond nodes represent the key miRNAs; up regulated genes are marked in green; down regulated genes are marked in red.

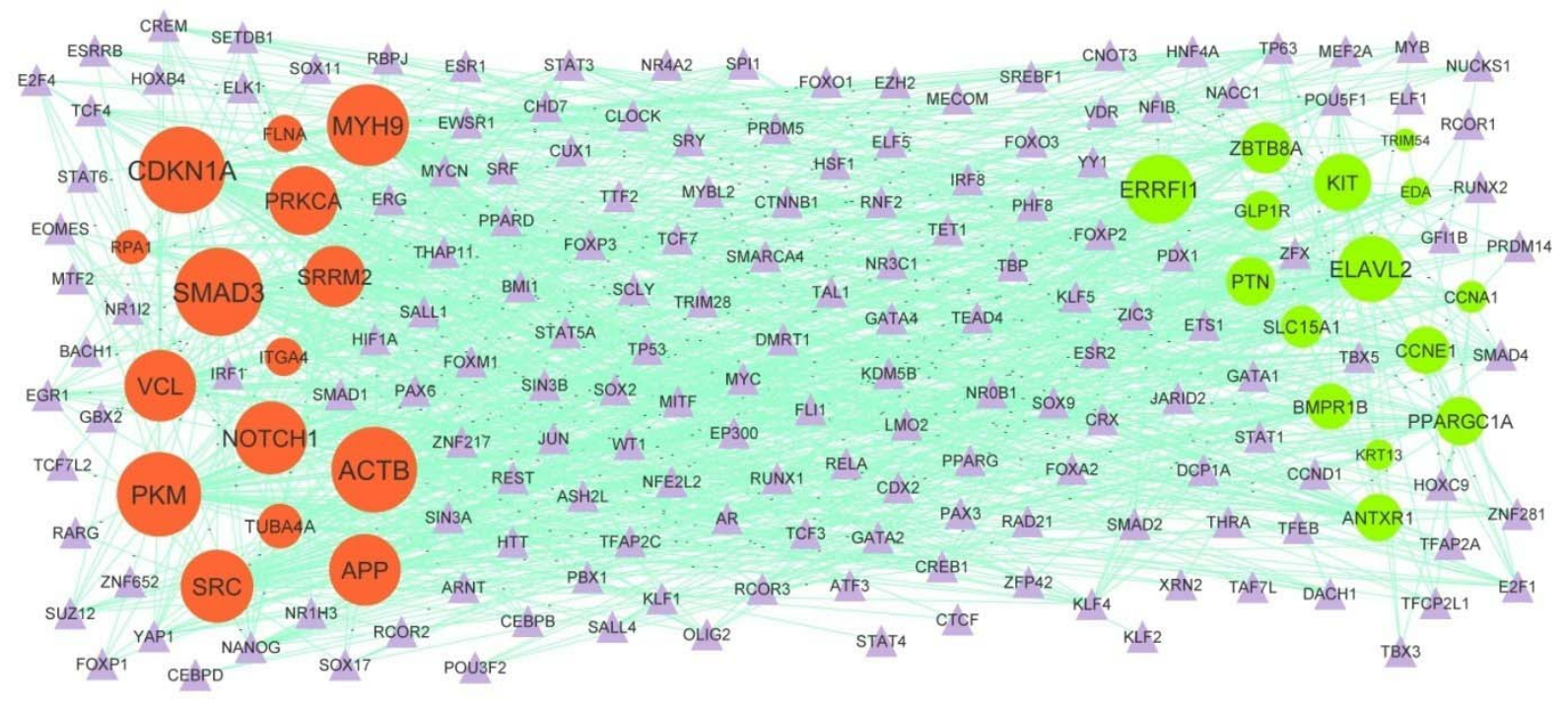

Fig. 6. Target gene - TF regulatory network between target genes. The purple color triangle nodes represent the key TFs; up regulated genes are marked in green; down regulated genes are marked in red. 

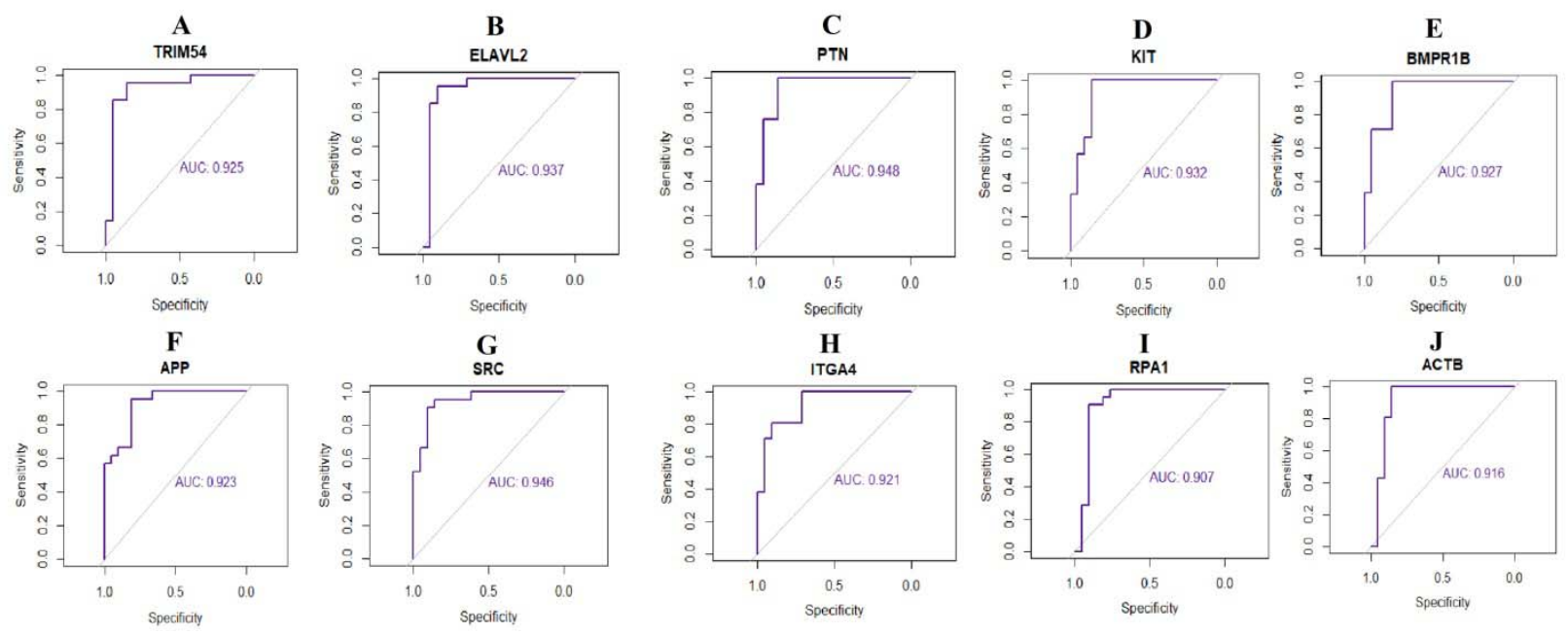

Fig. 7. ROC curve analyses of hub genes. A) TRIM54 B) ELAVL2 C) PTN D) KIT E) BMPR1B F) APP G) SRC H) ITGA4 I) RPA1 J) ACTB 